NASA/TP-2010-216213

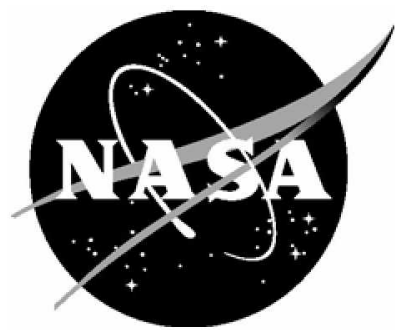

\title{
Faster and More Accurate Transport Procedures for HZETRN
}

Tony C. Slaba

Old Dominion University, Norfolk, Virginia

Steve R. Blattnig

Langley Research Center, Hampton, Virginia

Francis $F$. Badavi

Christopher Newport University, Newport News, Virginia 


\section{NASA STI Program ... in Profile}

Since its founding, NASA has been dedicated to the advancement of aeronautics and space science. The NASA scientific and technical information (STI) program plays a key part in helping NASA maintain this important role.

The NASA STI program operates under the auspices of the Agency Chief Information Officer. It collects, organizes, provides for archiving, and disseminates NASA's STI. The NASA STI program provides access to the NASA Aeronautics and Space Database and its public interface, the NASA Technical Report Server, thus providing one of the largest collections of aeronautical and space science STI in the world. Results are published in both non-NASA channels and by NASA in the NASA STI Report Series, which includes the following report types:

- TECHNICAL PUBLICATION. Reports of completed research or a major significant phase of research that present the results of NASA programs and include extensive data or theoretical analysis. Includes compilations of significant scientific and technical data and information deemed to be of continuing reference value. NASA counterpart of peerreviewed formal professional papers, but having less stringent limitations on manuscript length and extent of graphic presentations.

- TECHNICAL MEMORANDUM. Scientific and technical findings that are preliminary or of specialized interest, e.g., quick release reports, working papers, and bibliographies that contain minimal annotation. Does not contain extensive analysis.

- CONTRACTOR REPORT. Scientific and technical findings by NASA-sponsored contractors and grantees.
- CONFERENCE PUBLICATION. Collected papers from scientific and technical conferences, symposia, seminars, or other meetings sponsored or co-sponsored by NASA.

- SPECIAL PUBLICATION. Scientific, technical, or historical information from NASA programs, projects, and missions, often concerned with subjects having substantial public interest.

- TECHNICAL TRANSLATION. Englishlanguage translations of foreign scientific and technical material pertinent to NASA's mission.

Specialized services also include creating custom thesauri, building customized databases, and organizing and publishing research results.

For more information about the NASA STI program, see the following:

- Access the NASA STI program home page at http://www.sti.nasa.gov

- E-mail your question via the Internet to help@sti.nasa.gov

- Fax your question to the NASA STI Help Desk at 443-757-5803

- Phone the NASA STI Help Desk at 443-757-5802

- Write to: NASA STI Help Desk NASA Center for AeroSpace Information 7115 Standard Drive Hanover, MD 21076-1320 
NASA/TP-2010-216213

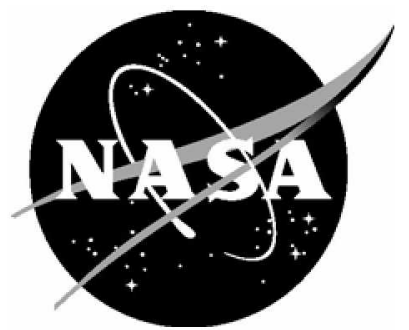

\section{Faster and More Accurate Transport Procedures for HZETRN}

Tony C. Slaba

Old Dominion University, Norfolk, Virginia

Steve R. Blattnig

Langley Research Center, Hampton, Virginia

Francis $F$. Badavi

Christopher Newport University, Newport News, Virginia

National Aeronautics and

Space Administration

Langley Research Center

Hampton, Virginia 23681-2199 
Available from:

NASA Center for AeroSpace Information

7115 Standard Drive

Hanover, MD 21076-1320

443-757-5802 


\section{Abbreviations}

A

BRYNTRN

$E$-grid

GCR

$\mathrm{GeV}$

HZETRN

$\mathrm{MeV}$

SPE
Atomic mass unit

BaRYoN TRaNsport

Energy grid

Galactic Cosmic Rays

Giga electron Volt

High charge (Z) and Energy TRaNsport

Mega electron Volt

Solar Particle Event 


\section{Contents}

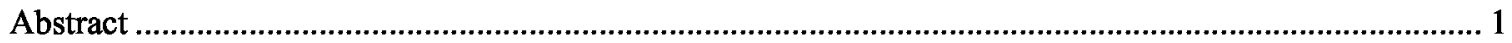

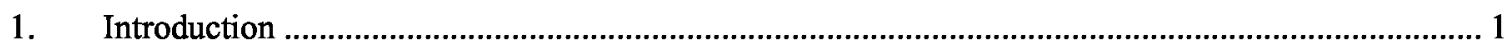

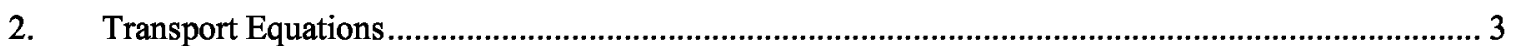

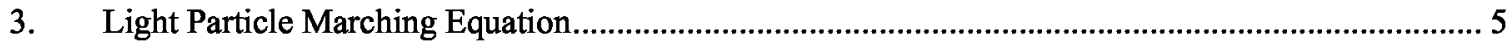

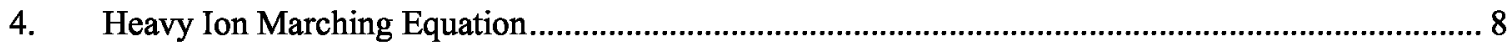

5. Existing Light Particle Marching Equation Numerical Methods............................................... 11

6. New Light Particle Marching Equation Numerical Methods..................................................... 15

7. Heavy Ion Marching Equation Numerical Methods ......................................................... 19

8. Coupling Light Particle and Heavy Ion Solutions for GCR ...................................................20

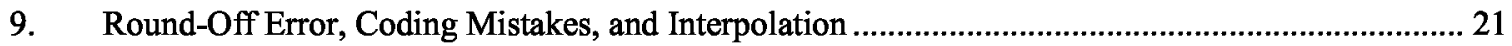

10. Convergence Study for Light Particle Transport in SPE Environments ..................................... 28

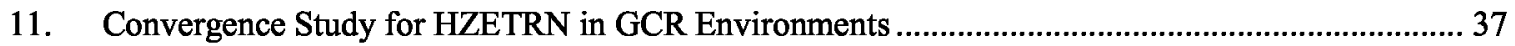

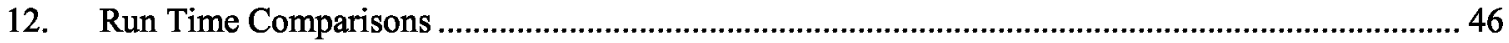

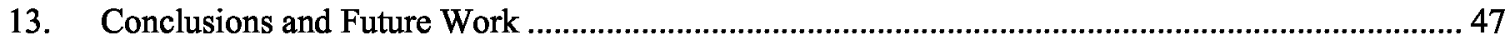

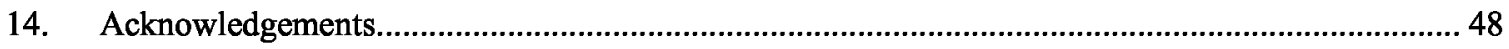

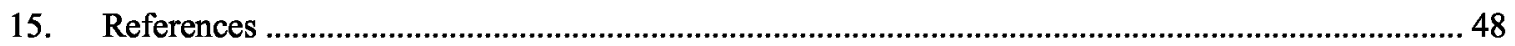

16. Appendix A - Inversion of Light Particle Transport Equation ................................................51

17. Appendix B - Inversion of Heavy Ion Transport Equation .................................................5 54 


\section{Figures}

1. Comparison of scaled proton range, $r / \nu_{j}$, and ${ }^{4} \mathrm{He}$ range,$r_{j}$, in aluminum. ....................................... 4

2. Process description of the existing light particle transport algorithm ........................................ 15

3. Process description of the new light particle transport algorithm................................................ 19

4. Integrated production cross section for a $1 \mathrm{AGeV}{ }^{4} \mathrm{He}$ ion producing ${ }^{3} \mathrm{He}$ and ${ }^{3} \mathrm{H}$ in aluminum.... 22

5. Secondary ${ }^{3} \mathrm{H}$ and ${ }^{3} \mathrm{He}$ fluences computed using single and double precision cross sections at 100 $\mathrm{g} / \mathrm{cm}^{2}$ in aluminum exposed to the ${ }^{4} \mathrm{He}$ component of the 1977 solar minimum GCR spectrum. ..... 23

6. Proton fluences computed with and without the maximum fragment energy coding error at $\mathbf{1 0 0}$ $\mathrm{g} / \mathrm{cm}^{2}$ in aluminum exposed to the August 1972 King SPE.......................................................... 24

7. Proton fluences computed with and without the maximum fragment energy and proton elastic coding errors at $100 \mathrm{~g} / \mathrm{cm}^{2}$ in aluminum exposed to the August $1972 \mathrm{King}$ SPE...................................... 25

8. Proton fluences computed with and without the maximum fragment energy and proton elastic coding errors at $100 \mathrm{~g} / \mathrm{cm}^{2}$ in water exposed to the August $1972 \mathrm{King}$ SPE............................................25

9. ${ }^{4} \mathrm{He}$ and secondary ${ }^{2} \mathrm{H}$ flux spectra computed with and without the spectral width error in $100 \mathrm{~g} / \mathrm{cm}^{2}$ of aluminum exposed to the 1977 solar minimum GCR environment................................................ 26

10. Comparison of old and new interpolation routines in $2 \mathrm{~g} / \mathrm{cm}^{2}$ of aluminum exposed to a modified form of the August 1972 King SPE.

11. Dose equivalent versus depth in aluminum exposed to the August 1972 King SPE computed with both transport algorithms and all discretization parameters..

12. Dose equivalent versus depth in water exposed to the August 1972 King SPE computed with both transport algorithms and all discretization parameters...................................................................... 30

13. Dose equivalent versus energy grid-size at $100 \mathrm{~g} / \mathrm{cm}^{2}$ in aluminum exposed to the August 1972 King SPE. Both transport algorithms were used with three different step-sizes.

14. Dose equivalent versus energy grid-size at $100 \mathrm{~g} / \mathrm{cm}^{2}$ in water exposed to the August $1972 \mathrm{King}$ SPE. Both transport algorithms were used with three different step-sizes. ...................................... 31

15. Dose equivalent versus step-size at $100 \mathrm{~g} / \mathrm{cm}^{2}$ in aluminum exposed to the August $1972 \mathrm{King}$ SPE. Both transport algorithms were used with six different energy grids. ........................................... 31

16. Dose equivalent versus step-size at $100 \mathrm{~g} / \mathrm{cm}^{2}$ in water exposed to the August $1972 \mathrm{King}$ SPE. Both transport algorithms were used with six different energy grids....................................................... 32

17. Discretization error for dose equivalent as a function of depth for the light particle transport algorithms in aluminum exposed to the August 1972 King SPE.

18. Discretization error for dose equivalent as a function of depth for the light particle transport algorithms in water exposed to the August 1972 King SPE. ........................................................... 33

19. Dose equivalent by particle type using the converged results at $100 \mathrm{~g} / \mathrm{cm}^{2}$ in aluminum exposed to the August 1972 King SPE.

20. Dose equivalent by particle type using the converged results at $100 \mathrm{~g} / \mathrm{cm}^{2}$ in water exposed to the August 1972 King SPE.

21. Discretization error (\%) caused by target fragments with energy less than $50 \mathrm{AMeV}$ in aluminum exposed to the August 1972 King SPE.

22. Dose equivalent versus depth in aluminum exposed to the 1977 solar minimum GCR computed with both transport algorithms and all discretization parameters............................................................ 38

23. Dose equivalent versus depth in water exposed to the 1977 solar minimum GCR computed with both transport algorithms and all discretization parameters.

24. Dose equivalent from heavy ions versus energy grid-size at $100 \mathrm{~g} / \mathrm{cm}^{2}$ in aluminum exposed to the 1977 solar minimum GCR environment. Five different steps-sizes were used. ................................. 39

25. Dose equivalent from heavy ions versus energy grid-size at $100 \mathrm{~g} / \mathrm{cm}^{2}$ in water exposed to the 1977 solar minimum GCR environment. Five different steps-sizes were used.

26. Dose equivalent from heavy ions versus step-size at $100 \mathrm{~g} / \mathrm{cm}^{2}$ in aluminum exposed to the 1977 solar minimum GCR environment. All of the different energy grids were used.

27. Dose equivalent from heavy ions versus step-size at $100 \mathrm{~g} / \mathrm{cm}^{2}$ in water exposed to the 1977 solar minimum GCR environment. All of the different energy grids were used.

28. Discretization error for dose equivalent as a function of depth for the heavy ion transport algorithm in aluminum exposed to the 1977 solar minimum GCR environment. White space included so vertical axis matches Figure 29. 
29. Discretization error for dose equivalent as a function of depth for the heavy ion transport algorithm in aluminum exposed to the 1977 solar minimum GCR environment.

30. Dose equivalent versus energy grid-size at $100 \mathrm{~g} / \mathrm{cm}^{2}$ in aluminum exposed to the 1977 solar minimum GCR environment. Both transport algorithms were used with three different step-sizes. . 43

31. Dose equivalent versus energy grid-size at $100 \mathrm{~g} / \mathrm{cm}^{2}$ in aluminum exposed to the 1977 solar minimum GCR environment. Both transport algorithms were used with three different step-sizes. 43

32. Dose equivalent versus step-size at $100 \mathrm{~g} / \mathrm{cm}^{2}$ in aluminum exposed to the 1977 solar minimum GCR environment. Both transport algorithms were used with six different energy grids................. 44

33. Dose equivalent versus step-size at $100 \mathrm{~g} / \mathrm{cm}^{2}$ in aluminum exposed to the 1977 solar minimum GCR environment. Both transport algorithms were used with six different energy grids.

34. Discretization error (\%) for dose equivalent as a function of depth for both algorithms in aluminum exposed to the 1977 solar minimum GCR environment.

35. Discretization error (\%) for dose equivalent as a function of depth for both algorithms in water exposed to the 1977 solar minimum GCR environment. 


\section{Tables}

1. Number of interpolations per step in the old and new light particle transport algorithms.

2. Dose at $100 \mathrm{~g} / \mathrm{cm}^{2}$ in aluminum and water exposed to August $1972 \mathrm{King}$ SPE and 1977 solar minimum GCR. The units for the SPE results are cGy, and the units for the GCR results are cGy/day......

3. Discretization error (\%) for dose equivalent computed with the old and new light particle transport algorithms at various depths in aluminum and water exposed to the August 1972 King SPE.

Significant figures shown to clarify magnitudes.

4. Discretization error (\%) for dose computed with the old and new light particle transport algorithms at various depths in aluminum and water exposed to the August 1972 King SPE. Significant figures shown to clarify magnitudes.

5. Discretization error (\%) for the heavy ion contribution to dose equivalent computed with the heavy ion transport algorithm at various depths in aluminum and water exposed to the 1977 solar minimum GCR. Significant figures shown to clarify magnitudes.

6. Discretization error (\%) for the heavy ion contribution to dose computed with the heavy ion transport algorithm at various depths in aluminum and water exposed to the 1977 solar minimum GCR. Significant figures shown to clarify magnitudes.

7. Total discretization error (\%) for dose equivalent computed with both algorithms at various depths in aluminum and water exposed to the 1977 solar minimum GCR. Significant figures shown to clarify magnitudes.

8. Total discretization error (\%) for dose computed with both algorithms at various depths in aluminum and water exposed to the 1977 solar minimum GCR. Significant figures shown to clarify magnitudes.

9. Single layer run time comparisons for old and new algorithms in SPE and GCR environments. The reduction factor is the ratio of the old method run-time to the new method run-time.

10. Two layer run time comparisons for old and new algorithms in SPE and GCR environments. The reduction factor is the ratio of the old method run-time to the new method run-time.

11. Three layer run time comparisons for old and new algorithms in SPE and GCR environments. The reduction factor is the ratio of the old method run-time to the new method run-time. 
Intentionally left blank 


\begin{abstract}
The deterministic transport code HZETRN was developed for research scientists and design engineers studying the effects of space radiation on astronauts and instrumentation protected by various shielding materials and structures. In this work, several aspects of code verification are examined. First, a detailed derivation of the light particle $(A \leq 4)$ and heavy ion $(A>4)$ numerical marching algorithms used in HZETRN is given. References are given for components of the derivation that already exist in the literature and discussions are given for details that have been neglected in the past. The present paper provides a complete description of the numerical methods currently used in the code and is identified as a key component of the verification process. Next, a new numerical method for light particle transport is presented, and improvements to the heavy ion transport algorithm are discussed. A summary of various coding errors discovered while implementing the new method is also given, and the impact of these errors on previously predicted exposure quantities is shown. Finally, a coupled convergence study is conducted by refining the discretization parameters (step-size and energy grid-size). From this study, it is clearly shown that past efforts in quantifying the numerical error in HZETRN were hindered by single precision calculations and computational resources. It is also determined that almost all of the discretization error in HZETRN is caused by the use of discretization parameters that violate a numerical convergence criterion related to charged target fragments below $50 \mathrm{AMeV}$. Total discretization errors are given for the old and new algorithms to $100 \mathrm{~g} / \mathrm{cm}^{2}$ in aluminum and water, and the improved accuracy of the new numerical methods is demonstrated. Run time comparisons are given between the old and new algorithms for three applications in which HZETRN is commonly used. The new algorithms are found to be almost 100 times faster for solar particle event simulations and almost 10 times faster for galactic cosmic ray simulations. The improved algorithms will be implemented in all future versions of HZETRN.
\end{abstract}

\title{
1. Introduction
}

As human exploration beyond Earth's orbit into radiation environments where measured data are sparse and testing is difficult, models will be heavily relied upon to make decisions regarding vehicle design and mission planning. This reliance on model results requires a systematic effort of verification, validation, and uncertainty quantification. Verification is the process of determining that a computational model accurately represents the underlying mathematical model and its solution; validation is the process of determining if the underlying mathematical model accurately represents physical reality, and uncertainty quantification is the process of identifying all relevant sources of uncertainties and quantifying their impact on the inputs and outputs of the model [NASA 2008]. This paper addresses verification of the radiation transport code HZETRN (High charge (Z) and Energy TRaNsport) [Wilson et al. 1991, 1995, 2006; Slaba et al. 2010] with a focus on documentation, improving efficiency and stability, and quantifying discretization error through convergence testing.

Documentation is a critical component of verification [Roy 2005] and has been emphasized in the NASA standard for modeling and simulation tools [NASA 2008]. While there have been many papers published that describe the transport model and physical parameters [Wilson et al. 1975, 1977, 1986, 1991, 2005,2006 ] as well as the marching algorithms and numerical methods [Wilson et al. 1989, 1991, 2005, 2006; Lamkin et al. 1992, 1994; Shinn et al. 1991] used in HZETRN, various gaps still exist in the documentation. For example, many of the mathematical details associated with inverting the Boltzmann integro-differential equation into a Volterra type integral equation have been neglected in the literature. Since the inversion depends on scaling conventions and physical arguments that are specific to the onedimensional space radiation transport problem, these mathematical details are a necessary component of code documentation. In this paper, we first give a detailed review and derivation of the existing light particle (n, p, ${ }^{2} \mathrm{H},{ }^{2} \mathrm{H},{ }^{3} \mathrm{He},{ }^{4} \mathrm{He}$ ) and heavy ion $(A>4)$ marching algorithms used in HZETRN. This will include all of the details associated with recasting the integro-differential equations as Volterra type 
integral equations and all of the physical and numerical approximations. References are given for components of the derivation that already exist in the literature, and discussions are given for details that have been neglected in the past. As a result of the review, a numerical convergence criterion is identified that, to the authors' knowledge, has yet to be documented or examined. It is shown later in the paper that the discretization parameters (spatial step-size and energy grid-size) commonly used in HZETRN violate this criterion and cause a systematic under-prediction of light charged target fragments below $50 \mathrm{AMeV}$.

This detailed derivation and review of the numerical methods also resulted in the development of a new light particle marching algorithm that is almost 100 times faster than its predecessor for solar particle event (SPE) simulations. Though computational efficiency has long been a core feature of HZETRN, there were certain applications for which the existing algorithms resulted in long run-times. For example, consider the interpolation or ray-by-ray methods used to compute mass averaged quantities in human phantoms exposed to space radiation. Interpolation methods are quite fast once the interpolation database has been generated, but it takes the current code over seven hours to generate a detailed database on a single processor. Similarly, the ray-by-ray method can take over 20 hours to compute the mass averaged particle fluence spectra at a single point in the body on a 192 core cluster. Approximately 1000 body points have been identified by Slaba et al. [2009] as sufficient for computing whole body effective dose in human phantoms exposed to SPE and galactic cosmic rays (GCR); this would indicate a significant computational cost. To help reduce these run-times and increase code efficiency, we present a new numerical method for the light particle marching algorithm that reduces the required number of interpolations and removes the need for integral fluence to be calculated at each step. The new method is shown to be almost 100 times faster for solar particle event (SPE) simulations and almost 10 times faster for galactic cosmic ray (GCR) simulations. The accuracy of the new methods is discussed later in the report.

Controlling round-off error and identifying coding errors is another important point of code verification. Though previous convergence studies [Shinn et al. 1991; Slaba et al. 2010] and benchmark comparisons [Wilson et al. 1988, 1991, 2005, 2006; Heinbockel et al. 2009a, 2009b] would indicate that round-off error is of little concern, such comparisons were generally made at moderate shielding depths where round-off errors are assumed to be small. However, as HZETRN is increasingly used in atmospheric, lunar, or Martian surface applications with large material thicknesses $\left(>50 \mathrm{~g} / \mathrm{cm}^{2}\right)$, round-off error could be a major concern and needs to be investigated. Therefore, we also discuss the impact of round-off error and coding errors in the existing marching algorithms in HZETRN. Selected light particle cross sections are calculated in single and double precision, and the impact of round-off error in the single precision calculations is shown to be large in certain cases. In the process of modifying HZETRN to enable double precision calculations, various coding errors were also discovered that have non-negligible effects on particle fluence spectra, dose, and dose equivalent. The coding errors are discussed and resolved. The interpolation routine [Wilson et al. 1995] frequently used in the transport algorithms is also examined, and a new routine is developed that is faster, has improved extrapolation procedures, and has the capability of interpolating around certain discontinuities. The improved code stability attained by using double precision calculations and removing the various coding errors is clearly shown.

In many computational models or algorithms, continuous variables are discretized to reduce a differential equation into an algebraic expression that is evaluated numerically. The algorithm is said to converge if the numerical solutions reach an asymptotic limit as the discretization parameters approach zero. In order to show convergence and quantify discretization error, the discretization parameters are refined several times and the differences between the various solutions are compared. Such studies are often referred to as convergence tests. As part of a larger verification and validation effort, configuration controlled convergence tests are created which can be rerun when significant changes are made to the codes. The ability to rerun such tests will help prevent the introduction of errors into the code as modifications are made in the future. In HZETRN, the spatial variable $x$ and energy variable $E$ are discretized. Two convergence tests have previously been published [Shinn et al. 1991; Slaba et al. 2010]; however, those tests were primarily focused on verifying code stability and were limited by computational resources. In the first analysis, Shinn et al. [1991] conducted a coupled convergence test in both space and energy. However, only two step-sizes $\left(h=0.5 \mathrm{~g} / \mathrm{cm}^{2}\right.$ and $\left.h=1.0 \mathrm{~g} / \mathrm{cm}^{2}\right)$ and two energy grids $(N=30$ and $N=60$, where $N$ is the number of grid points) were considered; only nucleons were transported, and all calculations were in single precision. No attempt was made to quantify discretization error, and numerical convergence was not clearly demonstrated. In the more recent analysis, it was determined that at least 100 energy grid points are needed to control energy discretization error [Slaba et al. 2010]. It will be shown here that even step-sizes of $h=0.01 \mathrm{~g} / \mathrm{cm}^{2}$ can result in moderate errors for low energy target fragments 
with small residual ranges. Neither the codes nor the results were configuration managed in these previous studies. Thus, a more detailed convergence analysis is necessary. We conduct a convergence study in both step-size and energy grid-size. The spatial discretization parameter, $h$, is reduced by factors of 2 from its common value of $0.5 \mathrm{~g} / \mathrm{cm}^{2}$ down to $2^{-11} \mathrm{~g} / \mathrm{cm}^{2}$. Similarly, the number of energy grid points is increased by factors of $\sim 1.5$ from 100 up to 753. Particle fluence, dose, and dose equivalent values are then computed at various depths in aluminum and water slabs exposed to SPE and GCR environments using the old and new transport algorithms and each of the discretization parameters. The resulting data are used to show that the new algorithms reach an asymptotic solution as the discretization parameters are refined. The improved accuracy of the new methods is also clearly demonstrated. Discretization errors are also given for the discretization parameters commonly used in HZETRN $\left(h=0.5 \mathrm{~g} / \mathrm{cm}^{2}\right.$ with 100 energy grid points). These errors are expressed as percent differences from the converged numerical solutions obtained with the finest discretization parameters.

\section{Transport Equations}

The Boltzmann transport equation with the continuous slowing down and straight ahead approximations is given as [Wilson et al. 1991]

$$
\bar{B}\left[\phi_{j}(x, E)\right]=\sum_{k} \int_{E}^{\infty} \sigma_{j k}\left(E, E^{\prime}\right) \phi_{k}\left(x, E^{\prime}\right) d E^{\prime}
$$

with the linear differential operator

$$
\bar{B}\left[\phi_{j}(x, E)\right] \equiv\left[\frac{\partial}{\partial x}-\frac{1}{A_{j}} \frac{\partial}{\partial E} S_{j}(E)+\sigma_{j}(E)\right] \phi_{j}(x, E),
$$

and boundary condition

$$
\phi_{j}(0, E)=f_{j}(E)
$$

In equations (1)-(3), $\phi_{j}(x, E)$ is the fluence of type $j$ particles at depth $x$ with kinetic energy $E, A_{j}$ is the atomic mass number of a type $j$ particle, $S_{j}(E)$ is the stopping power of a type $j$ ion with kinetic energy $E$, $\sigma_{j}(E)$ is the total macroscopic cross section for a type $j$ particle with kinetic energy $E$, and $\sigma_{j k}\left(E, E^{\prime \prime}\right)$ is the macroscopic production cross section for interactions in which a type $k$ particle with kinetic energy $E^{\prime}$ produce a type $j$ particle with kinetic energy $E$. The summation limits in equation (1) will be discussed shortly. The boundary condition spectrum, $f_{j}(E)$, is considered to be a known function over a broad energy spectrum.

Consider the continuous slowing down operator

$$
\left[\frac{1}{A_{j}} \frac{\partial}{\partial E} S_{j}(E)\right] \phi_{j}(x, E)
$$

which represents the rate at which charged particles lose energy as they interact with the electron clouds in the target media. Although atomic interactions cause charged particles to lose energy in discrete increments as they pass through a material, there are a sufficient number of these interactions in a unit path length to justify a continuous approximation [Wilson et al. 1991]. It is advantageous to approximate this term by considering the relation from Bethe stopping power theory [Bethe et al. 1930]

$$
\nu_{k} r_{k} \approx \nu_{j} r_{j}
$$


where $\nu_{j}=Z_{j}^{2} / A_{j}$ with $Z_{j}$ being the atomic charge of a type $j$ particle, and the range of a type $j$ ion, $r_{j}$, is given by the range-energy relationship

$$
r_{j} \equiv A_{j} \int_{0}^{E} \frac{d E^{\prime}}{S_{j}\left(E^{\prime}\right)}
$$

From equation (5) and (6), it is clear that the proton range and stopping power can be approximated as

$$
\begin{gathered}
r \approx \nu_{j} r_{j}, \\
\nu_{j} S(E) \approx \frac{1}{A_{j}} S_{j}(E) .
\end{gathered}
$$

The approximation in equation (7) is depicted in Figure 1 and is less accurate at low energy ( $<10 \mathrm{AMeV})$ or low residual range.

Equation (8) allows the transport equation to be written as

$$
B\left[\phi_{j}(x, E)\right]=\sum_{k} \int_{E}^{\infty} \sigma_{j k}\left(E, E^{\prime}\right) \phi_{k}\left(x, E^{\prime}\right) d E^{\prime},
$$

with

$$
B\left[\phi_{j}(x, E)\right] \equiv\left[\frac{\partial}{\partial x}-\nu_{j} \frac{\partial}{\partial E} S(E)+\sigma_{j}(E)\right] \phi_{j}(x, E)
$$

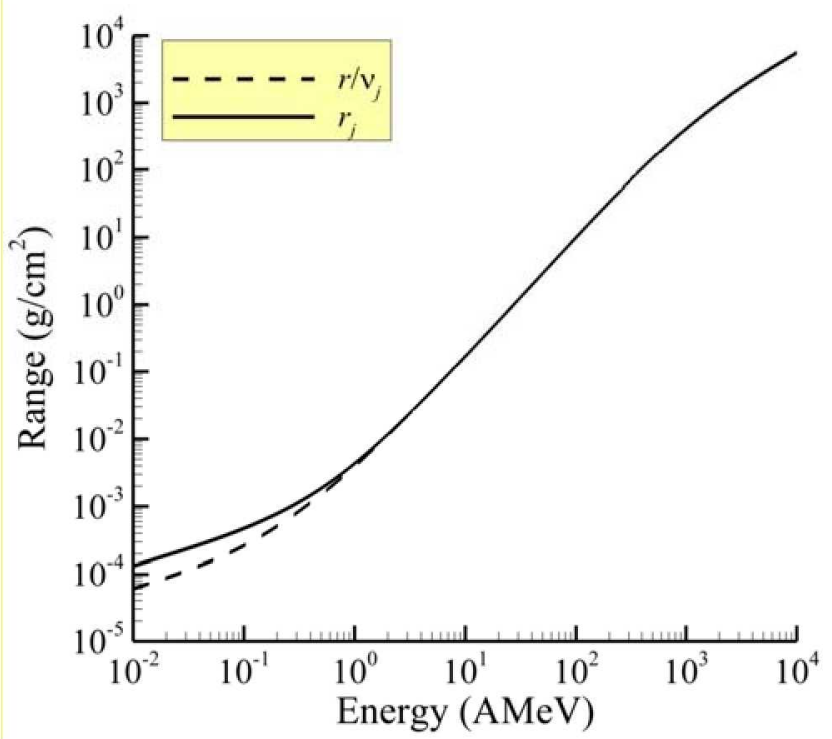

Figure 1. Comparison of scaled proton range, $r / \nu_{j}$, and ${ }^{4} \mathrm{He}$ range,$r_{j}$, in aluminum. 
The motivation, justification, and physical implications associated with approximating each ion stopping power with a scaled proton stopping power has been discussed in detail elsewhere [Wilson et al. 2006]. Ultimately, the approximation allows significant numerical simplifications, as will be shown later.

There are two paths taken (for light particles and heavy ions) in developing numerical procedures for equation (9). For heavy ions, it is noted that projectile fragments have energy and direction very near that of the projectile, while target fragments are produced nearly isotropically with low energy and travel only a short distance before being absorbed [Wilson et al., 1977, 1986, 1991, 1995, 2006]. The approximate decoupling of target and projectile fragments is discussed in detail by Wilson et al. [1977, 1991, 1995] and suggests that target fragments can be neglected in the heavy ion transport procedure (their contribution to dose is approximately accounted for after the transport procedure). The equal velocity assumption for heavy ions can be expressed in the production cross section as [Shinn et al. 1992]

$$
\sigma_{j k}\left(E, E^{\prime}\right)=\sigma_{j k}\left(E^{\prime}\right) \delta\left(E-E^{\prime}\right)
$$

where $\sigma_{j k}(E)$ is the production cross section for interactions in which a type $k$ particle with kinetic energy $E$ produce a type $j$ particle. In this case, equation (9) becomes

$$
B\left[\phi_{j}(x, E)\right]=\sum_{k} \sigma_{j k}(E) \phi_{k}(x, E) .
$$

The absence of target fragments in the heavy ion transport procedure allows one to take the summation in equation (9) over all projectiles with mass greater than that of the fragment. If all the transported particles are ordered according to mass, then equation (9) can be succinctly written as

$$
B\left[\phi_{j}(x, E)\right]=\sum_{k>j} \sigma_{j k}(E) \phi_{k}(x, E),
$$

which is the transport equation found in Wilson et al. [1986, 1991, 1995, 2006] and will be referred to as the heavy ion transport equation. The upper summation limit in equation (13) can vary, but it is common to use no fewer than 59 ions. See Cucinotta et al. [2006] for a discussion of isotope selection.

Alternatively, for light particles (n, $p,{ }^{2} \mathrm{H},{ }^{2} \mathrm{H},{ }^{3} \mathrm{He},{ }^{4} \mathrm{He}$ ) both projectile and target fragments are included in the transport procedure. The broad energy distribution in collision events also indicates that the equal velocity assumption in equation (11) cannot be used. In this case, no simplifications to equation (9) are used, and the summation is taken over all light particles. Hereafter, equation (9) will be referred to as the light particle transport equation which includes both neutrons and light ions. It should be noted that for SPE environments with a negligible heavy ion component, only the solution to the light particle transport equation is required. For GCR environments, there are both heavy ion and light ion components, and solutions to the light particle and heavy ion transport equations must be evaluated simultaneously. The coupling of these two equations will be discussed later.

\section{Light Particle Marching Equation}

The following formulation of the light particle marching equation was taken from Wilson et al. [1975, 1989, 1991, 2006], Shinn et al. [1991], Lamkin et al. [1992, 1994], Cucinotta [1993], and Slaba et al. [2010]. Further references in this section will be given only for mathematical techniques and physical arguments.

To develop a numerical marching procedure for the light particle transport equation, it is necessary to invert the differential operator on the left hand side of equation (9) by using the method of characteristics [Haberman 1989]. To do this, define the scaled quantities

$$
\psi_{j}(x, r) \equiv \bar{\nu}_{j} S(E) \phi_{j}(x, E)
$$

and 


$$
s_{j k}\left(r, r^{\prime}\right) \equiv S(E) \sigma_{j k}\left(E, E^{\prime}\right)
$$

where the proton range, $r$, is defined in terms of the proton stopping power, $S(E)$, by the range-energy relationship

$$
r \equiv \int_{0}^{E} \frac{d E^{\prime}}{S\left(E^{\prime}\right)}
$$

and the modified scaling parameter is given by

$$
\bar{\nu}_{j} \equiv\left\{\begin{array}{cc}
1, & j=n \\
\nu_{j} & , j \neq n
\end{array} .\right.
$$

The quantity $\bar{\nu}_{j}$ is used in fluence scaling to avoid a trivial solution for the neutrons. Notice that we have used the scaled proton range in equation (14) to allow the function $\psi_{j}(x, r)$ to be defined over the common proton range, $r$, for each particle type $j$.

In terms of the scaled quantities introduced in equations (14) and (15), the integrals on the right hand side of the light particle transport equation become

$$
\begin{aligned}
\sum_{k} \int_{E}^{\infty} \sigma_{j k}\left(E, E^{\prime}\right) \phi_{k}\left(x, E^{\prime}\right) d E^{\prime} & =\sum_{k} \int_{E}^{\infty}\left(\frac{1}{S(E)} s_{j k}\left(r, r^{\prime}\right)\right)\left(\frac{1}{\bar{\nu}_{k} S\left(E^{\prime}\right)} \psi_{k}\left(x, r^{\prime}\right)\right) d E^{\prime} \\
& =\sum_{k} \frac{1}{\bar{\nu}_{k} S(E)} \int_{r}^{\infty} s_{j k}\left(r, r^{\prime}\right) \psi_{k}\left(x, r^{\prime}\right) d r^{\prime}
\end{aligned}
$$

where the differential has been transformed using

$$
d r^{\prime}=\frac{1}{S\left(E^{\prime}\right)} d E^{\prime}
$$

Now, note that

$$
\frac{\partial \psi_{j}}{\partial r}=\frac{\partial \psi_{j}}{\partial E} \frac{d E}{d r}=\bar{\nu}_{j} S(E) \frac{\partial}{\partial E}\left[S(E) \phi_{j}(x, E)\right]
$$

so that the differential operator on the left hand side of the light particle transport equation can be expressed in terms of the scaled quantities as

$$
\begin{aligned}
{\left[\frac{\partial}{\partial x}-\nu_{j} \frac{\partial}{\partial E} S(E)+\sigma_{j}(E)\right] \phi_{j}(x, E) } & =\frac{\partial \phi_{j}}{\partial x}-\nu_{j} \frac{\partial}{\partial E}\left[S(E) \phi_{j}\right]+\sigma_{j}(E) \phi_{j} \\
& =\frac{1}{\bar{\nu}_{j} S(E)} \frac{\partial \psi_{j}}{\partial x}-\frac{\nu_{j}}{\bar{\nu}_{j} S(E)} \frac{\partial \psi_{j}}{\partial r}+\frac{1}{\bar{\nu}_{j} S(E)} \sigma_{j} \psi_{j} \\
& =\left[\frac{\partial}{\partial x}-\nu_{j} \frac{\partial}{\partial r}+\sigma_{j}(E)\right] \frac{1}{\bar{\nu}_{j} S(E)} \psi_{j}(x, r)
\end{aligned}
$$

where the functional dependencies of the scaled and unscaled fluence have been suppressed in certain places for brevity. Equations (18) and (21) are now combined to give the light particle transport equation in terms of the scaled quantities as 


$$
\left[\frac{\partial}{\partial x}-\nu_{j} \frac{\partial}{\partial r}+\sigma_{j}(E)\right] \frac{1}{\bar{\nu}_{j} S(E)} \psi_{j}(x, r)=\sum_{k} \frac{1}{\bar{\nu}_{k} S(E)} \int_{r}^{\infty} s_{j k}\left(r, r^{\prime}\right) \psi_{k}\left(x, r^{\prime}\right) d r^{\prime},
$$

which simplifies to give the final scaled form

$$
\left[\frac{\partial}{\partial x}-\nu_{j} \frac{\partial}{\partial r}+\sigma_{j}(r)\right] \psi_{j}(x, r)=\sum_{k} \frac{\bar{\nu}_{j}}{\bar{\nu}_{k}} \int_{r}^{\infty} s_{j k}\left(r, r^{\prime}\right) \psi_{k}\left(x, r^{\prime}\right) d r^{\prime}
$$

The term $\sigma_{j}(E)$ has been replaced with $\sigma_{j}(r)$, since for a given value of $r$, equation (16) can be inverted to determine $E$.

Equation (23) is inverted (see Appendix A) using the method of characteristic to obtain the Volterra type integral equation

$$
\begin{aligned}
\psi_{j}(x, r) & =e^{-\beta_{j}(r, x)} \psi_{j}\left(0, r+\nu_{j} x\right) \\
& +\sum_{k} \frac{\bar{\nu}_{j}}{\bar{\nu}_{k}} \int_{0}^{x} \int_{r+\nu_{j} x^{\prime}}^{\infty} e^{-\beta_{j}\left(r, x^{\prime}\right)} s_{j k}\left(r+\nu_{j} x^{\prime}, r^{\prime}\right) \psi_{k}\left(x-x^{\prime}, r^{\prime}\right) d r^{\prime} d x^{\prime},
\end{aligned}
$$

with the integrating factor

$$
\beta_{j}(r, x) \equiv \int_{0}^{x} \sigma_{j}\left(r+\nu_{j} t\right) d t
$$

Equation (24) can be easily written as a marching procedure in terms of the step-size, $h$, as

$$
\begin{aligned}
\psi_{j}(x+h, r) & =e^{-\beta_{j}(r, h)} \psi_{j}\left(x, r+\nu_{j} h\right) \\
& +\sum_{k} \frac{\bar{\nu}_{j}}{\bar{\nu}_{k}} \int_{0}^{h} \int_{r+\nu_{j} x^{\prime}}^{\infty} e^{-\beta_{j}\left(r, x^{\prime}\right)} s_{j k}\left(r+\nu_{j} x^{\prime}, r^{\prime}\right) \psi_{k}\left(x+h-x^{\prime}, r^{\prime}\right) d r^{\prime} d x^{\prime} .
\end{aligned}
$$

We now seek to approximate the differential fluence in the integrand of equation (26). Define

$$
Q_{j k}\left(x, r+\nu_{j} x^{\prime}\right) \equiv \int_{r+\nu_{j} x^{\prime}}^{\infty} e^{-\beta_{j}\left(r, x^{\prime}\right)} s_{j k}\left(r+\nu_{j} x^{\prime}, r^{\prime}\right) \psi_{k}\left(x+h-x^{\prime}, r^{\prime}\right) d r^{\prime}
$$

then

$$
Q_{j k}\left(x, r+\nu_{j} x^{\prime}\right)=Q_{j k}(x, r)+O\left(x^{\prime}\right)
$$

The $O\left(x^{k}\right)$ notation indicates that the remaining terms in the polynomial expansion are of degree $k$ or greater. The exponential attenuation term can also be expanded as

$$
e^{-\beta_{j}\left(r, x^{\prime}\right)}=1+O\left(x^{\prime}\right)
$$

where the expansions $e^{-\beta}=1-\beta+O\left(\beta^{2}\right)$ and $\beta_{j}\left(r, x^{\prime}\right) \approx x^{\prime} \sigma_{j}(r)$ have been used. Substitution of equations (27)-(29) into equation (26) produces 


$$
\psi_{j}(x+h, r)=e^{-\beta_{j}(r, h)} \psi_{j}\left(x, r+\nu_{j} h\right)+\sum_{k} \frac{\bar{\nu}_{j}}{\bar{\nu}_{k}} \int_{0}^{h} Q_{j k}(x, r)+O\left(x^{\prime}\right) d x^{\prime},
$$

or

$$
\psi_{j}(x+h, r)=e^{-\beta_{j}(r, h)} \psi_{j}\left(x, r+\nu_{j} h\right)+O(h)
$$

Equation (31), without the $O(h)$ term is the exact solution to the homogeneous equation

$$
\left[\frac{\partial}{\partial x}-\nu_{j} \frac{\partial}{\partial r}+\sigma_{j}(r)\right] \psi_{j}(x, r)=0
$$

The homogeneous equation neglects secondary particle production through nuclear interactions and accounts only for the slowing down of particles due to atomic interactions and the loss of particles due to nuclear absorption. If the step-size is taken to be sufficiently small such that

$$
h \ll \frac{1}{\sigma_{j}(r)},
$$

(i.e. much less than the nuclear mean free path), then the local truncation error will be negligible as the particles will not have travelled far enough to suffer a nuclear collision [Wilson et al. 1991]. Equation (33) is the first convergence criterion and has been well documented in the literature [Wilson et al. 1975, 1989, 1991, 2006]. It is worth noting that nuclear mean free path lengths are on the order of many $\mathrm{g} / \mathrm{cm}^{2}$, while the step-sizes usually taken in HZETRN are less than $1 \mathrm{~g} / \mathrm{cm}^{2}$.

Equation (31) can be used to approximate the integrand in equation (26) by noting that [Wilson et al. $1975,1989,1991,2006]$

$$
\psi_{k}\left(x+h-x^{\prime}, r^{\prime}\right)=e^{-\beta_{k}\left(r^{\prime}, h-x^{\prime}\right)} \psi_{k}\left(x, r^{\prime}+\nu_{k}\left(h-x^{\prime}\right)\right)+O(h) .
$$

Substitution of equation (34) into equation (26) yields

$$
\begin{aligned}
\psi_{j}(x+h, r) & =e^{-\beta_{j}(r, h)} \psi_{j}\left(x, r+\nu_{j} h\right) \\
& +\sum_{k} \frac{\bar{\nu}_{j}}{\bar{\nu}_{k}} \int_{0}^{h} \int_{r+\nu_{j} x^{\prime}}^{\infty} e^{-\beta_{j}\left(r, x^{\prime}\right)-\beta_{k}\left(r^{\prime}, h-x^{\prime}\right)} s_{j k}\left(r+\nu_{j} x^{\prime}, r^{\prime}\right) \psi_{k}\left(x, r^{\prime}+\nu_{k}\left(h-x^{\prime}\right)\right) d r^{\prime} d x^{\prime} \\
& +O\left(h^{2}\right)
\end{aligned}
$$

where the $O\left(h^{2}\right)$ terms are small. This is the light particle marching equation given by Wilson et al. [2006] for which numerical procedures will be developed and studied later. Hereafter, the $O\left(h^{2}\right)$ will be assumed in the marching equations and not written.

\section{Heavy Ion Marching Equation}

The following derivation of the heavy ion marching equation was taken from Wilson et al. [1977, 1986, 1991, 1995, 2006] and Shinn et al. [1992]. Further references will only be given for mathematical techniques. Any variables and symbols used in this section that were defined in the previous section hold their respective definitions.

The heavy ion transport equation was given previously in equation (13) as 


$$
B\left[\phi_{j}(x, E)\right]=\sum_{k>j} \sigma_{j k}(E) \phi_{k}(x, E)
$$

Define the scaled heavy ion fluence

$$
\psi_{j}(x, r) \equiv \nu_{j} S(E) \phi_{j}(x, E)
$$

and replace $\sigma_{j k}(E)$ with $\sigma_{j k}(r)$ since, for a given $r$, equation (16) can be inverted to find $E$. Notice that the scaling in equation (37) is identical to the scaling used for the light particles in equation (14) except that we have used $\nu_{j}$ instead of $\bar{\nu}_{j}$. This minor change reflects the fact that we are now only transporting heavy charged particles for which $\nu_{j}>0$ and trivial scaling is not possible.

Following the procedure outlined in the previous section, equation (36) is written in terms of the scaled fluence as

$$
\left[\frac{\partial}{\partial x}-\nu_{j} \frac{\partial}{\partial r}+\sigma_{j}(r)\right] \psi_{j}(x, r)=\sum_{k>j} \frac{\nu_{j}}{\nu_{k}} \sigma_{j k}(r) \psi_{k}(x, r)
$$

Equation (38) is inverted (see Appendix B) using the method of characteristic to obtain the Volterra type integral equation

$$
\begin{aligned}
\psi_{j}(x, r)= & e^{-\beta_{j}(r, x)} \psi_{j}\left(0, r+\nu_{j} x\right) \\
& +\sum_{k>j} \frac{\nu_{j}}{\nu_{k}} \int_{0}^{x} e^{-\beta_{j}\left(r, x^{\prime}\right)} \sigma_{j k}\left(r+\nu_{j} x^{\prime}\right) \psi_{k}\left(x-x^{\prime}, r+\nu_{j} x^{\prime}\right) d x^{\prime}
\end{aligned}
$$

with the exponential term

$$
\beta_{j}(r, x)=\int_{0}^{x} \sigma_{j}\left(r+\nu_{j} t\right) d t
$$

Equation (39) can be easily written as a marching procedure in terms of the step-size, $h$, as

$$
\begin{aligned}
\psi_{j}(x+h, r)= & e^{-\beta_{j}(r, h)} \psi_{j}\left(x, r+\nu_{j} h\right) \\
& +\sum_{k>j} \frac{\nu_{j}}{\nu_{k}} \int_{0}^{h} e^{-\beta_{j}\left(r, x^{\prime}\right)} \sigma_{j k}\left(r+\nu_{j} x^{\prime}\right) \psi_{k}\left(x+h-x^{\prime}, r+\nu_{j} x^{\prime}\right) d x^{\prime}
\end{aligned}
$$

As before, use the $O(h)$ homogenous solution [Wilson et al. 2006]

$$
\psi_{k}\left(x+h-x^{\prime}, r+\nu_{j} x^{\prime}\right)=e^{-\beta_{k}\left(r+\nu_{j} x^{\prime}, h_{h}-x^{\prime}\right)} \psi_{k}\left(x, r+\nu_{j} x^{\prime}+\nu_{k}\left(h-x^{\prime}\right)\right)+O(h),
$$

to obtain

$$
\begin{aligned}
\psi_{j}(x+h, r)= & e^{-\beta_{j}(r, h)} \psi_{j}\left(x, r+\nu_{j} h\right) \\
& +\sum_{k>j} \frac{\nu_{j}}{\nu_{k}} \int_{0}^{h} e^{-\beta_{j}\left(r, x^{\prime}\right)-\beta_{k}\left(r+\nu_{j} x^{\prime}, h-x^{\prime}\right)} \sigma_{j k}\left(r+\nu_{j} x^{\prime}\right) \psi_{k}\left(x, r+\nu_{j} x^{\prime}+\nu_{k}\left(h-x^{\prime}\right)\right) d x^{\prime} \\
& +O\left(h^{2}\right)
\end{aligned}
$$


From here, approximate the integral over $x^{\prime}$ from 0 to $h$ by expanding the production cross section and particle fluence in a Taylor series [Wade 2000] as

$$
\begin{gathered}
\sigma_{j k}\left(r+\nu_{j} x^{\prime}\right)=\sigma_{j k}\left(r+\nu_{j} h / 2\right)+O\left(x^{\prime}\right), \\
\psi_{k}\left(x, r+\nu_{j} x^{\prime}+\nu_{k}\left(h-x^{\prime}\right)\right)=\psi_{k}\left(x, r+\left(\nu_{j}+\nu_{k}\right) h / 2\right)+O\left(x^{\prime}\right),
\end{gathered}
$$

and obtain

$$
\begin{aligned}
& \psi_{j}(x+h, r)=e^{-\beta_{j}(r, h)} \psi_{j}\left(x, r+\nu_{j} h\right) \\
& \quad+\sum_{k>j} \frac{\nu_{j}}{\nu_{k}} \sigma_{j k}\left(r+\nu_{j} h / 2\right) \psi_{k}\left(x, r+\left(\nu_{j}+\nu_{k}\right) h / 2\right) \int_{0}^{h} e^{-\beta_{j}\left(r, x^{\prime}\right)-\beta_{k}\left(r+\nu_{j} x^{\prime}, h-x^{\prime}\right)} d x^{\prime} \\
& \quad+O\left(h^{2}\right) .
\end{aligned}
$$

The remaining integral is similarly handled by considering the following Taylor expansions for the exponential terms

$$
\begin{aligned}
\beta_{j}\left(r, x^{\prime}\right) & =\int_{0}^{x^{\prime}} \sigma_{j}\left(r+\nu_{j} t\right) d t \\
& \approx \int_{0}^{x^{\prime}} \sigma_{j}\left(r+\nu_{j} h / 2\right) d t \\
& =\sigma_{j}\left(r+\nu_{j} h / 2\right) x^{\prime}+O\left(\left(x^{\prime}\right)^{2}\right), \\
\beta_{k}\left(r+\nu_{j} x^{\prime}, h-x^{\prime}\right) & =\int_{0}^{h-x^{\prime}} \sigma_{k}\left(r+\nu_{j} x^{\prime}+\nu_{k} t\right) d t \\
& \approx \int_{0}^{h-x^{\prime}} \sigma_{k}\left(r+\nu_{j} x^{\prime}+\nu_{k} h / 2\right) d t \\
& =\sigma_{j}\left(r+\nu_{j} x^{\prime}+\nu_{k} h / 2\right)\left(h-x^{\prime}\right) \\
& \approx \sigma_{j}\left(r+\left(\nu_{j}+\nu_{k}\right) h / 2\right)\left(h-x^{\prime}\right)+O\left(\left(x^{\prime}\right)^{2}\right) .
\end{aligned}
$$

The integral is now evaluated as

$$
\begin{aligned}
\int_{0}^{h} e^{-\beta_{j}\left(r, x^{\prime}\right)-\beta_{k}\left(r+\nu_{j} x^{\prime}, h-x^{\prime}\right)} d x^{\prime} & \approx \int_{0}^{h} e^{-\sigma_{j}\left(r+\nu_{j} h / 2\right) x^{\prime}-\sigma_{k}\left(r+\left(\nu_{j}+\nu_{k}\right) h / 2\right)\left(h-x^{\prime}\right)} d x^{\prime} \\
& =\frac{e^{-\sigma_{j}\left(r+\nu_{j} h / 2\right) h}-e^{-\sigma_{k}\left(r+\left(\nu_{j}+\nu_{k}\right) h / 2\right) h}}{\sigma_{k}\left(r+\left(\nu_{j}+\nu_{k}\right) h / 2\right)-\sigma_{j}\left(r+\nu_{j} h / 2\right)}+O\left(h^{2}\right) .
\end{aligned}
$$

The final marching procedure is given by

$$
\begin{aligned}
& \psi_{j}(x+h, r)=e^{-\beta_{j}(r, h)} \psi_{j}\left(x, r+\nu_{j} h\right) \\
& +\sum_{k>j} \frac{\nu_{j}}{\nu_{k}} \sigma_{j k}\left(r+\nu_{j} h / 2\right) \psi_{k}\left(x, r+\left(\nu_{j}+\nu_{k}\right) h / 2\right) \\
& \quad \times \frac{e^{-\sigma_{j}\left(r+\nu_{j} h / 2\right) h}-e^{-\sigma_{k}\left(r+\left(\nu_{j}+\nu_{k}\right) h / 2\right) h}}{\sigma_{k}\left(r+\left(\nu_{j}+\nu_{k}\right) h / 2\right)-\sigma_{j}\left(r+\nu_{j} h / 2\right)}+O\left(h^{2}\right),
\end{aligned}
$$


where the $O\left(h^{2}\right)$ terms are small. This is the heavy ion marching equation given by Wilson et al. [2006] for which numerical procedures will be developed and studied later. Hereafter, the $O\left(h^{2}\right)$ will be assumed in the marching equations and not written.

\section{Existing Light Particle Marching Equation Numerical Methods}

In this section, the numerical methods developed to evaluate equation (35) are reviewed and the error analysis found in the literature for those methods is summarized. The following notation and terminology will be used extensively throughout the remainder of the report. An energy grid ( $E$-grid) refers to a discrete set of energy values distributed in some manner between a minimum energy value, $E_{\min }$, and a maximum energy value, $E_{\max }$. The $i^{\text {th }}$ component of the $E$-grid is denoted as $E_{i}$. A range grid ( $r$-grid) refers to a discrete set of proton range values distributed in some manner between a minimum range value, $r_{\min }$, and a maximum range value, $r_{\max }$. The $i^{\text {th }}$ component of the $r$-grid is denoted as $r_{i}$. The number of grid points in a grid, or grid-size, is denoted by $N$. It will be assumed that all grid indexing is from $i=1$ to $i=N$. Equal-log spacing is also used extensively; the $i^{\text {th }}$ component of an equal-log spaced $E$-grid is evaluated as

$$
E_{i}=10^{\log \left(E_{\min }\right)+\Delta_{E}(i-1)},
$$

where

$$
\Delta_{E}=\frac{\log \left(E_{\max }\right)-\log \left(E_{\min }\right)}{N-1}
$$

and the logarithms are base 10 . Note that an energy grid can be converted to a range grid, and vice-versa, using equation (16).

In Section 3, we derived the light particle marching equation (35) as

$$
\begin{aligned}
& \psi_{j}(x+h, r)=e^{-\beta_{j}(r, h)} \psi_{j}\left(x, r+\nu_{j} h\right) \\
& \quad+\sum_{k} \frac{\bar{\nu}_{j}}{\bar{\nu}_{k}} \int_{0}^{h} \int_{r+\nu_{j} x^{\prime}}^{\infty} e^{-\beta_{j}\left(r, x^{\prime}\right)-\beta_{k}\left(r^{\prime}, h-x^{\prime}\right)} s_{j k}\left(r+\nu_{j} x^{\prime}, r^{\prime}\right) \psi_{k}\left(x, r^{\prime}+\nu_{k}\left(h-x^{\prime}\right)\right) d r^{\prime} d x^{\prime} .
\end{aligned}
$$

It is clear from equation (53) that the quantity $\psi_{j}\left(x, r+\nu_{j} h\right)$ will likely have to be evaluated for range values not on the prescribed $r$-grid. Wilson et al. [1991] and Lamkin et al. [1992] have shown that a log-log cubic Lagrange interpolating polynomial provides sufficient accuracy with a minimal number of grid points. The terminology "log-log" refers to taking the logarithm of both the dependent and independent variables prior to interpolating. The interpolated value is then exponentiated to adjust for the initial logarithm. It has been shown that higher order Lagrange polynomials offer little more in accuracy, and higher order splines can introduce uncontrollable oscillatory behavior that is problematic in iterative marching procedures.

First simplify the integral over $x^{\prime}$ from 0 to $h$ by using a modified single point midpoint rule for integrable functions $a(x), b(x)$, and $c(x)$ of the form

$$
\int_{0}^{h} a(x) b(x) c(x) d x \approx a(h / 2)\left[\int_{0}^{h} b(x) d x\right] c(h / 2) .
$$

This approximation will be accurate if the functions $a(x)$ and $c(x)$ are nearly constant over the interval $[0, h]$. When applied to equation (53), this approximation yields [Wilson et al. 1991, 2006] 


$$
\begin{aligned}
& \psi_{j}(x+h, r)=e^{-\beta_{j}(r, h)} \psi_{j}\left(x, r+\nu_{j} h\right) \\
& \quad+\sum_{k} \frac{\bar{\nu}_{j}}{\bar{\nu}_{k}} \int_{r+\nu_{j} h / 2}^{\infty} e^{-\beta_{j}(r, h / 2)-\beta_{k}\left(r^{\prime}, h / 2\right)}\left[\int_{0}^{h} s_{j k}\left(r+\nu_{j} x^{\prime}, r^{\prime}\right) d x^{\prime}\right] \psi_{k}\left(x, r^{\prime}+\nu_{k} h / 2\right) d r^{\prime}
\end{aligned}
$$

It is clear that the modified midpoint rule has decoupled the source integrals in equation (53); however, the approximation will only be accurate if the step-size is sufficiently small to satisfy

$$
\varepsilon\left(r+\nu_{j} h / 2\right) \approx \varepsilon(r)
$$

or

$$
h \ll r / \nu_{j} \approx r_{j}
$$

Equation (57) is the second convergence criterion. Recall that the first criterion was identified previously in equation (33) and required the step-size to be smaller than the nuclear mean free path length. To the authors' knowledge, this second criterion has never been explicitly stated or addressed in the literature. Note that the range of a $100 \mathrm{MeV}$ proton in aluminum is $\sim 10 \mathrm{~g} / \mathrm{cm}^{2}$, so a step-size of $0.5 \mathrm{~g} / \mathrm{cm}^{2}$ will be sufficient in this approximation. However, a $10 \mathrm{MeV}$ proton in aluminum will travel $\sim 0.17 \mathrm{~g} / \mathrm{cm}^{2}$ before coming to rest, and a $1 \mathrm{MeV}$ proton in aluminum will travel $\sim 0.004 \mathrm{~g} / \mathrm{cm}^{2}$ before coming to rest. This indicates that step-sizes of $0.5 \mathrm{~g} / \mathrm{cm}^{2}$ and $1.0 \mathrm{~g} / \mathrm{cm}^{2}$ studied previously [Shinn et al. 1991] do not accurately transport low energy target fragments. It will be shown later that even step-sizes near $0.01 \mathrm{~g} / \mathrm{cm}^{2}$ will systematically under-predict the source integral by neglecting the particle production from projectiles with low ranges near that of fragments. This approximation will be tested in detail when the coupled energy grid and step-size convergence study is conducted. fragments

Continue simplifying the light particle transport equation by noticing that for charged particle

$$
\begin{aligned}
\int_{0}^{h} s_{j k}\left(r+\nu_{j} x^{\prime}, r^{\prime}\right) d x^{\prime} & =\frac{1}{\nu_{j}} \int_{r}^{r+\nu_{j} h} s_{j k}\left(u, r^{\prime}\right) d u \\
& =\frac{1}{\nu_{j}}\left[\int_{0}^{r+\nu_{j} h} s_{j k}\left(u, r^{\prime}\right) d u-\int_{0}^{r} s_{j k}\left(u, r^{\prime}\right) d u\right] \\
& =\frac{1}{\nu_{j}}\left[\int_{0}^{\varepsilon\left(r+\nu_{j} h\right)} \sigma_{j k}\left(E, E^{\prime}\right) d E-\int_{0}^{\varepsilon(r)} \sigma_{j k}\left(E, E^{\prime}\right) d E\right] \\
& \equiv F_{j k}^{\Delta}\left(r, r^{\prime} ; h\right) .
\end{aligned}
$$

The term $\varepsilon(r)$ is the energy associated with the proton range $r$, and for neutron fragments, denoted by $j=$ $n$,

$$
\begin{aligned}
\int_{0}^{h} s_{n k}\left(r+\nu_{n} x^{\prime}, r^{\prime}\right) d x^{\prime} & =\int_{0}^{h} s_{n k}\left(r, r^{\prime}\right) d x^{\prime} \\
& =h s_{n k}\left(r, r^{\prime}\right) \\
& \equiv F_{n k}^{\Delta}\left(r, r^{\prime} ; h\right)
\end{aligned}
$$

The light particle transport equation is now written as

$$
\begin{aligned}
\psi_{j}(x+h, r) & =e^{-\beta_{j}(r, h)} \psi_{j}\left(x, r+\nu_{j} h\right) \\
& +\sum_{k} \frac{\bar{\nu}_{j}}{\bar{\nu}_{k}} \int_{r+\nu_{j} h / 2}^{\infty} e^{-\beta_{j}(r, h / 2)-\beta_{k}\left(r^{\prime}, h / 2\right)} F_{j k}^{\Delta}\left(r, r^{\prime} ; h\right) \psi_{k}\left(x, r^{\prime}+\nu_{k} h / 2\right) d r^{\prime} .
\end{aligned}
$$


The exponential attenuation terms are simplified using [Shinn et al. 1991; Lamkin et al. 1992]

$$
\begin{gathered}
e^{-\beta_{j}(r, h)}=e^{-\int_{0}^{h} \sigma_{j}\left(r+\nu_{j} t\right) d t} \approx e^{-\sigma_{j}(r) h}, \\
e^{-\beta_{j}(r, h / 2)}=e^{-\int_{0}^{h / 2} \sigma_{j}\left(r+\nu_{j} t\right) d t} \approx e^{-\sigma_{j}(r) h / 2},
\end{gathered}
$$

and

$$
e^{-\beta_{k}\left(r^{\prime}, h / 2\right)}=e^{-\int_{0}^{h / 2} \sigma_{k}\left(r^{\prime}+\nu_{k} t\right) d t} \approx e^{-\sigma_{k}\left(r^{\prime}\right) h / 2} .
$$

Equations (61)-(63) are exact for neutrons and have been shown [Shinn et al. 1991; Lamkin et al. 1992] to produce negligible errors for charged particles with step-sizes up to $h=0.5 \mathrm{~g} / \mathrm{cm}^{2}$. The light particle transport equation is now reduced to

$$
\begin{aligned}
\psi_{j}(x+h, r)= & e^{-\sigma_{j}(r) h} \psi_{j}\left(x, r+\nu_{j} h\right) \\
& +\sum_{k} \frac{\bar{\nu}_{j}}{\bar{\nu}_{k}} \int_{r+\nu_{j} h / 2}^{\infty} e^{-\sigma_{j}(r) h / 2-\sigma_{k}\left(r^{\prime}\right) h / 2} F_{j k}^{\Delta}\left(r, r^{\prime} ; h\right) \psi_{k}\left(x, r^{\prime}+\nu_{k} h / 2\right) d r^{\prime}
\end{aligned}
$$

Now consider another one-point quasi-midpoint rule for integrable functions $f(x)$ and $g(x)$ integrated over a closed interval $[a, b]$

$$
\int_{a}^{b} f(x) g(x) d x \approx f(\bar{x}) \int_{a}^{b} g(x) d x
$$

where $\bar{x}=(a+b) / 2$. This approximation will be accurate if $f(x)$ is nearly constant over the interval $[a, b]$. The composite quasi-midpoint rule is obtained by extending the approximation over several subintervals within a given region

$$
\int_{a}^{b} f(x) g(x) d x \approx \sum_{i=1}^{N-1} f\left(\bar{x}_{i}\right) \int_{x_{i}}^{x_{i+1}} g(x) d x
$$

where $x_{1}=a, x_{N}=b$, and $\bar{x}_{i}=\left(x_{i+1}+x_{i}\right) / 2$. This approximation is similar to the multi-group method commonly used in nuclear reactor transport theory and requires the sub-intervals $\left[x_{i}, x_{i+1}\right]$ to be sufficiently small such that $f(x)$ is nearly constant over the sub-interval [Marchuk et al. 1986]. The composite quasi-midpoint rule in equation (66) can be applied to the integral in equation (64) by first making a simple change of variables $\left(r^{\prime \prime}=r^{\prime}-\nu_{j} h / 2\right)$ so that the light particle transport equation becomes

$$
\begin{aligned}
& \psi_{j}(x+h, r)=e^{-\sigma_{j}(r) h} \psi_{j}\left(x, r+\nu_{j} h\right) \\
& \quad+\sum_{k} \frac{\bar{\nu}_{j}}{\bar{\nu}_{k}} \int_{r}^{\infty} e^{-\sigma_{j}(r) h / 2-\sigma_{k}\left(r^{\prime \prime}+\nu_{j} h / 2\right) h / 2} F_{j k}^{\Delta}\left(r, r^{\prime \prime}+\nu_{j} h / 2 ; h\right) \psi_{k}\left(x, r "+\left(\nu_{j}+\nu_{k}\right) h / 2\right) d r^{\prime \prime}
\end{aligned}
$$

Define the integral fluence as 


$$
\Psi_{j}(x, r)=\int_{r}^{\infty} \psi_{j}\left(x, r^{\prime \prime}\right) d r^{\prime \prime}
$$

so that for a given $r_{i}$, equation (67) is evaluated as

$$
\begin{aligned}
\psi_{j}\left(x+h, r_{i}\right)= & e^{-\sigma_{j}\left(r_{i}\right) h} \psi_{j}\left(x, r_{i}+\nu_{j} h\right) \\
& +\sum_{k} \frac{\bar{\nu}_{j}}{\bar{\nu}_{k}} \sum_{m=i}^{N-1} e^{-\sigma_{j}\left(r_{i}\right) h / 2-\sigma_{k}\left(r_{m}\right) h / 2} F_{j k}^{\Delta}\left(r_{i}, \bar{r}_{m}+\nu_{j} h / 2 ; h\right) \\
& \times\left[\Psi_{j}\left(x, r_{m}+\left(\nu_{j}+\nu_{k}\right) h / 2\right)-\Psi_{j}\left(x, r_{m+1}+\left(\nu_{j}+\nu_{k}\right) h / 2\right)\right],
\end{aligned}
$$

where $\bar{r}_{m} \equiv\left(r_{m+1}+r_{m}\right) / 2$. Slaba et al. [2010] have recently shown this approximation to be poor for neutron elastic interactions in which the energy loss between the pre-collision and post-collision neutron is very small. They have adjusted the neutron elastic component, $F_{n n}^{\Delta, e l}$, so that it is now evaluated as

$$
F_{n n}^{\Delta, e l}\left(r_{i}, \bar{r}_{m} ; h\right)=h S(E)\left\langle\sigma_{n n}^{e l}\left(r_{i}, \bar{r}_{m}\right)\right\rangle
$$

where

$$
\left\langle\sigma_{n n}^{e l}\left(r_{i}, \bar{r}_{m}\right)\right\rangle=\frac{1}{\varepsilon\left(r_{m+1}\right)-\varepsilon\left(r_{m}\right)} \int_{\varepsilon\left(r_{m}\right)}^{\varepsilon\left(r_{m+1}\right)} \sigma_{n n}^{e l}\left(\varepsilon\left(r_{i}\right), E^{\prime}\right) d E^{\prime},
$$

is the average value of the differential neutron elastic production cross section over the interval $\left[r_{m}, r_{m+1}\right]$.

Note that another approximation has also been made, namely

$$
e^{-\sigma_{k}\left(\bar{r}_{n}+\nu_{j} h / 2\right) h / 2} \approx e^{-\sigma_{k}\left(r_{n}\right) h / 2}
$$

Equations (68)-(72) define the light particle marching procedure used in HZETRN and tested in this report. A final point to make about the light particle marching procedure is related to manner in which it is coded (ie. the main algorithm used to evaluate the equation). Figure 2 gives a general process description of the light particle marching algorithm. The required inputs are the initial and final positions as well as the boundary flux/fluence at the initial position. The next step is to determine the maximum step-size $\left(h_{\max }<\right.$ 0.5 ) and integer $P$ such that $P$ iterations of the light particle marching equation will propagate the fluences from the initial position to the final position. Once the constant step-size has been determined, the exponential attenuation term and production cross sections are computed for all values of $r$ on the $r$-grid. These values are then used repeatedly within the main loop. Once the main loop is entered, the integral fluence must be computed, and the differential and integral fluences must be interpolated for each particle and each range value.

The most computationally expensive portion of the calculation is the cross section calculation, which has been outlined in a bold dashed line in Figure 2. The integral fluence, interpolation, and source term calculations are not as computationally expensive, but are repeated several times within the loop and are therefore important. They have been outlined with a smaller dashed line. It should also be noted that in this algorithm, all of the computations are carried out in single precision. More will be said about this later. 


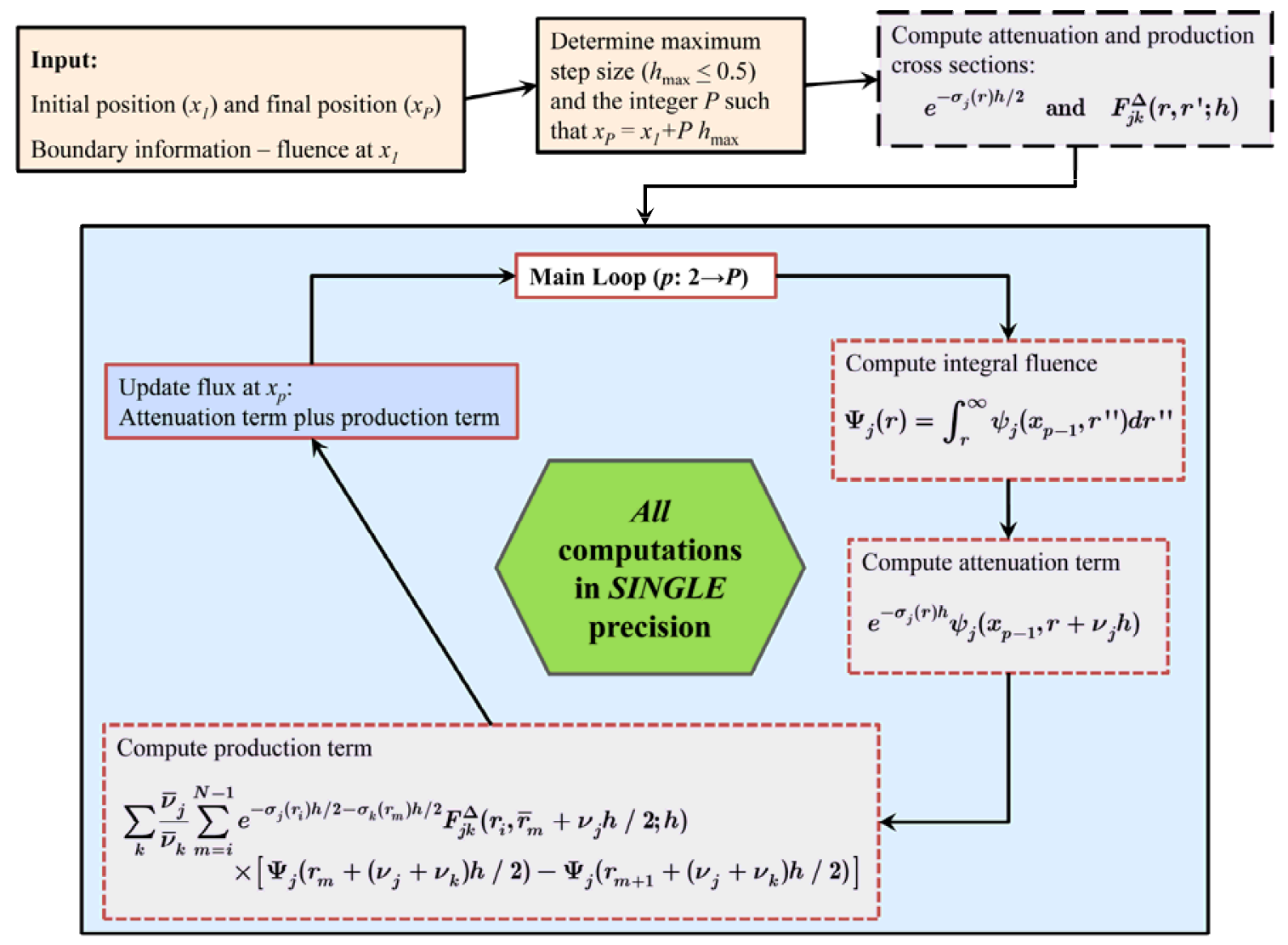

Figure 2. Process description of the existing light particle transport algorithm.

\section{New Light Particle Marching Equation Numerical Methods}

In this section, new numerical methods are presented to evaluate the light particle marching equation. The approximations used in the previous section to get from equation (53) to equation (67) are all used in this section; therefore, begin with equation (67) and make the substitution

$$
r^{\prime}=r^{\prime \prime}+\left(\nu_{j}+\nu_{k}\right) h / 2 \text {, }
$$

so that the light particle transport equation becomes

$$
\begin{aligned}
\psi_{j}(x+h, r) & =e^{-\sigma_{j}(r) h} \psi_{j}\left(x, r+\nu_{j} h\right) \\
& +\sum_{k} \int_{r}^{\infty} e^{-\sigma_{j}(r) h / 2-\sigma_{k}\left(r^{\prime}-\nu_{k} h / 2\right) h / 2} \bar{F}_{j k}^{\Delta}\left(r, r^{\prime}-\nu_{k} h / 2 ; h\right) \psi_{k}\left(x, r^{\prime}\right) d r^{\prime}
\end{aligned}
$$

where

$$
\bar{F}_{j k}^{\Delta}\left(r, r^{\prime} ; h\right) \equiv \frac{\bar{\nu}_{j}}{\bar{\nu}_{k}} F_{j k}^{\Delta}\left(r, r^{\prime} ; h\right)
$$

Now, suppose that the fluence in the integrand, $\psi_{k}\left(x, r^{\prime}\right)$, can be approximated by a linear combination of log-linear basis splines as 


$$
\psi_{k}\left(x, r^{\prime}\right) \approx \sum_{m=1}^{N} B_{m}^{(1)}\left(r^{\prime}\right) \psi_{k}\left(x, r_{m}\right)
$$

with the log-linear spline

$$
B_{m}^{(l)}(r)=\left\{\begin{array}{cl}
\ln \left(r / r_{m-1}\right) / \ln \left(r_{m} / r_{m-1}\right) & , r \in\left[r_{m-1}, r_{m}\right] \\
\ln \left(r_{m+1} / r\right) / \ln \left(r_{m+1} / r_{m}\right) & , r \in\left[r_{m}, r_{m+1}\right] \\
0 & , \text { otherwise }
\end{array}\right.
$$

Substitute equation (77) into equation (74) and get

$$
\begin{aligned}
& \psi_{j}(x+h, r)=e^{-\sigma_{j}(r) h} \psi_{j}\left(x, r+\nu_{j} h\right) \\
& \quad+\sum_{k} \sum_{m=1}^{N} \psi_{k}\left(x, r_{m}\right) \int_{r+\left(\nu_{j}+\nu_{k}\right) h / 2}^{\infty} e^{-\sigma_{j}(r) h / 2-\sigma_{k}\left(r^{\prime}-\nu_{k} h / 2\right) h / 2} \bar{F}_{j k}^{\Delta}\left(r, r^{\prime}-\nu_{k} h / 2 ; h\right) B_{m}^{(l)}\left(r^{\prime}\right) d r^{\prime}
\end{aligned}
$$

It is clear from equation (78) that the integrand and the integral no longer depend on the fluence or the depth in the material. In fact, the integral can now be treated as a constant matrix of production coefficients depending only on the step-size and the $r$-grid. If we evaluate equation (78) at the $i^{\text {th }} r$-grid value, then the production coefficients can be defined as

$$
a_{i m}(h) \equiv \int_{r_{i}+\left(\nu_{j}+\nu_{k}\right) h / 2}^{\infty} e^{-\sigma_{j}\left(r_{i}\right) h / 2-\sigma_{k}\left(r^{\prime}-\nu_{k} h / 2\right) h / 2} \bar{F}_{j k}^{\Delta}\left(r_{i}, r^{\prime}-\nu_{k} h / 2 ; h\right) B_{m}^{(1)}\left(r^{\prime}\right) d r^{\prime},
$$

so that the transport equation is now greatly simplified as

$$
\psi_{j}\left(x+h, r_{i}\right)=e^{-\sigma_{j}\left(r_{i}\right) h} \psi_{j}\left(x, r_{i}+\nu_{j} h\right)+\sum_{k} \sum_{m=1}^{N} a_{i m}(h) \psi_{k}\left(x, r_{m}\right)
$$

This approach casts the production integral as a linear combination of terms computed on the original $r$ grid requiring no further interpolation or integration.

The production coefficients, $a_{i m}$, are further simplified by noting that the basis spline, $B_{m}^{(l)}\left(r^{\prime}\right)$, is non-zero only in the region $\left[r_{m-1}, r_{m+1}\right]$. Therefore,

$$
a_{i m}(h)=\int_{r_{a}}^{r_{b}} e^{-\sigma_{j}\left(r_{i}\right) h / 2-\sigma_{k}\left(r^{\prime}-\nu_{k} h / 2\right) h / 2} \bar{F}_{j k}^{\Delta}\left(r_{i}, r^{\prime}-\nu_{k} h / 2 ; h\right) B_{m}^{(1)}\left(r^{\prime}\right) d r^{\prime},
$$

where

$$
\begin{gathered}
r_{a}=\max \left\{r_{i}+\left(\nu_{j}+\nu_{k}\right) h / 2, r_{m-1}\right\}, \\
r_{b}=\min \left\{r_{N}, r_{m+1}\right\},
\end{gathered}
$$

and $\min$ and $\max$ refer to the minimum and maximum values, respectively. For neutron elastic interactions, the upper limit has been adjusted to

$$
r_{b}=\min \left\{r_{N}, r_{m+1}, r_{\alpha}\right\},
$$


where $r_{\alpha}$ is the proton range associated with the energy $E_{\alpha}=\varepsilon\left(r_{i}\right) / \alpha_{T}, \varepsilon\left(r_{i}\right)$ is the energy associated with the proton range $r_{i}$, and $\alpha_{T}$ is the target dependent parameter given by

$$
\alpha_{T}=\left(\frac{A_{T}-1}{A_{T}+1}\right)^{2},
$$

where $A_{T}$ is the mass of the target nucleus. To understand where the term $r_{\alpha}$ was taken from, note that Wilson et al. [1991] have parameterized the neutron elastic production cross section to be non-zero in the energy region

$$
\alpha_{T} E^{\prime}<E<E^{\prime},
$$

where $E^{\prime}$ is the energy of the pre-collision neutron and $E$ is the energy of the post-collision neutron. This equation can also be expressed in terms of the pre-collision neutron energy as

$$
E<E^{\prime}<E / \alpha_{T}
$$

For heavy targets, in which $\alpha_{T} \rightarrow 1$, the non-zero region of the neutron elastic production cross section can become smaller than the spacing of the $E$-grid (or $r$-grid). Including the upper bound of the elastic cross section in the selection of the upper limit of integration will properly account for such occurrences.

Similarly, for quasi-elastic interactions involving ${ }^{4} \mathrm{He}$ projectiles, the lower limit of integration has been adjusted to

$$
r_{a}=\max \left\{r_{i}+\left(\nu_{j}+\nu_{k}\right) h / 2, r_{m-1}, r_{i}^{(B)}+h / 2\right\}
$$

where $r_{i}^{(B)}$ is the proton range associated with the energy $\varepsilon\left(r_{i}\right)+E_{B}$, and $E_{B}$ is the binding energy of the struck nucleus. To understand where the term $r_{i}^{(B)}+h / 2$ was taken from, recall that for ${ }^{4} \mathrm{He}$ quasi-elastic interactions, secondary ${ }^{4} \mathrm{He}$ ions have energy $E$ satisfying

$$
E<E^{\prime}-E_{B},
$$

where $E^{\prime}$ is the projectile energy and $E_{B}$ is the binding energy of the struck nucleus. In equation (81), the cumulative production cross section, $\bar{F}_{j k}^{\Delta}$, is evaluated at $r^{\prime}-\nu_{k} h / 2$. Since $\nu_{k}=1$ for ${ }^{4} \mathrm{He}$, the minimum projectile energy must be $\varepsilon\left(r_{i}^{(B)}+h / 2\right)$.

In order to further simplify the integral in equation (81), the exponential terms in the integrand are approximated as

$$
e^{-\sigma_{j}\left(r_{i}\right) h / 2-\sigma_{k}\left(r^{\prime}-\nu_{k} h / 2\right) h / 2} \approx e^{-\sigma_{j}\left(r_{i}\right) h / 2-\sigma_{k}(\bar{r}) h / 2},
$$

with $\bar{r} \equiv\left(r_{a}+r_{b}\right) / 2$. The approximation is valid since the exponential terms are slowly varying over the small interval $\left(r_{a}, r_{b}\right)$. In this case, the production coefficients become

$$
a_{i m}(h)=e^{-\sigma_{j}\left(r_{i}\right) h / 2-\sigma_{k}(\bar{r}) h / 2} \int_{r_{a}}^{r_{b}} \bar{F}_{j k}^{\Delta}\left(r_{i}, r^{\prime}-\nu_{k} h / 2 ; h\right) B_{m}^{(1)}\left(r^{\prime}\right) d r^{\prime},
$$


and are numerically evaluated using a 10-point Gauss-Legendre quadrature. For some reactions in which the cross sections are highly peaked or rapidly varying over the interval $\left(r_{a}, r_{b}\right)$, several subintervals are used to improve accuracy, and the quadrature is applied over each of the subintervals. For $n$ producing $n$ through elastic collisions, ten subintervals are used (100 quadrature points per integral); for $n$ producing $n$ through reactive interactions, five subintervals are used (50 quadrature points per integral); for ${ }^{4} \mathrm{He}$ producing ${ }^{4} \mathrm{He}$ through quasi-elastic interactions, five subintervals are used (50 quadrature points per integral). For all remaining reaction channels, two subintervals are used (20 quadrature points per integral).

Even though the numerical integrations are carried out over small subintervals of the $r$-grid, the production coefficients are still computationally expensive to calculate for a single $h$ value; therefore, they are approximated by using the log-linear interpolation

$$
a_{i m}(h) \approx \exp \left\{a_{i m}^{(l)}\left(h_{p+1}\right)+\left[a_{i m}^{(l)}\left(h_{p+1}\right)-a_{i m}^{(l)}\left(h_{p}\right)\right]\left(h^{(l)}-h_{p}^{(l)}\right) /\left(h_{p+1}^{(l)}-h_{p}^{(l)}\right)\right\} \equiv a_{i m}^{*},
$$

where $a_{i m}^{(l)} \equiv \log \left(a_{i m}\right)$ and $h^{(l)} \equiv \log (h)$. This requires the coefficients $a_{i m}(h)$ to be pre-computed for several values of $h$ from $0.0 \mathrm{~g} / \mathrm{cm}^{2}$ to $0.5 \mathrm{~g} / \mathrm{cm}^{2}$. The $h$-grid has been taken to be

$$
h_{p}=\{0.0,0.001,0.005,0.01,0.05,0.1,0.25,0.5\} \text {. }
$$

The final marching equation for light particles is now written as

$$
\psi_{j}\left(x+h, r_{i}\right)=e^{-\sigma_{j}\left(r_{i}\right) h} \psi_{j}\left(x, r_{i}+\nu_{j} h\right)+\sum_{k} \sum_{m=1}^{N} a_{i m}^{*} \psi_{k}\left(x, r_{m}\right)
$$

After reviewing the older light particle marching algorithm depicted in Figure 2, it was determined that the cross sections used in the exponential attenuation term could also be pre-computed and passed to the algorithm as an argument. This modification has been made in the new algorithm. A general process description of the revised light particle marching algorithm is depicted in Figure 3. The required inputs are the initial and final positions as well as the boundary flux/fluence at the initial position, the attenuation cross sections, and the production coefficients as a function of $h$. The next step is to determine the maximum step-size $\left(h_{\max }<0.5\right)$ and integer $P$ such that $P$ iterations of the light particle marching equation will propagate the fluences from the initial position to the final position. Once the constant step-size has been determined, the exponential attenuation term and the production coefficients are computed for all values of $r$ on the $r$-grid.

In the new algorithm, a significant improvement in efficiency is gained since the production coefficients are now obtained through a simple log-linear interpolation and the exponential attenuation terms are computed directly from the attenuation cross sections. Once the main loop is entered, only the interpolated values, $\psi_{j}\left(x, r+\nu_{j} h\right)$, must be computed at each iteration. No integral fluences need to be computed, and no interpolations over the integral fluences need to be calculated. The most computationally expensive portion of the calculation is now the interpolation and has therefore been given a dashed outline in Figure 3. The interpolation routine [Wilson et al. 1995] used in previous versions of HZETRN and BRYNTRN was reviewed and several modifications were made to improve efficiency and robustness. The updated routine still performs $\log$-log cubic interpolation with Lagrange polynomials but is more efficient and has improved logic for interpolation around certain discontinuities and extrapolations past $r_{\max }$. A comparison of the routines will be given later. It should also be noted that in the new algorithm, all of the computations are carried out in double precision; the need for double precision will be shown later.

The updated algorithm is much simpler and faster than its predecessor. An estimate of the improvements can be obtained by simply counting the number of interpolations in a single iteration of the light particle transport equation. For this exercise, recall that there are six particles to transport and assume there are $N$ grid points in the $r$-grid. The results are summarized in Table 1. It is clear the new algorithm requires several orders of magnitude fewer interpolations than the old algorithm. 


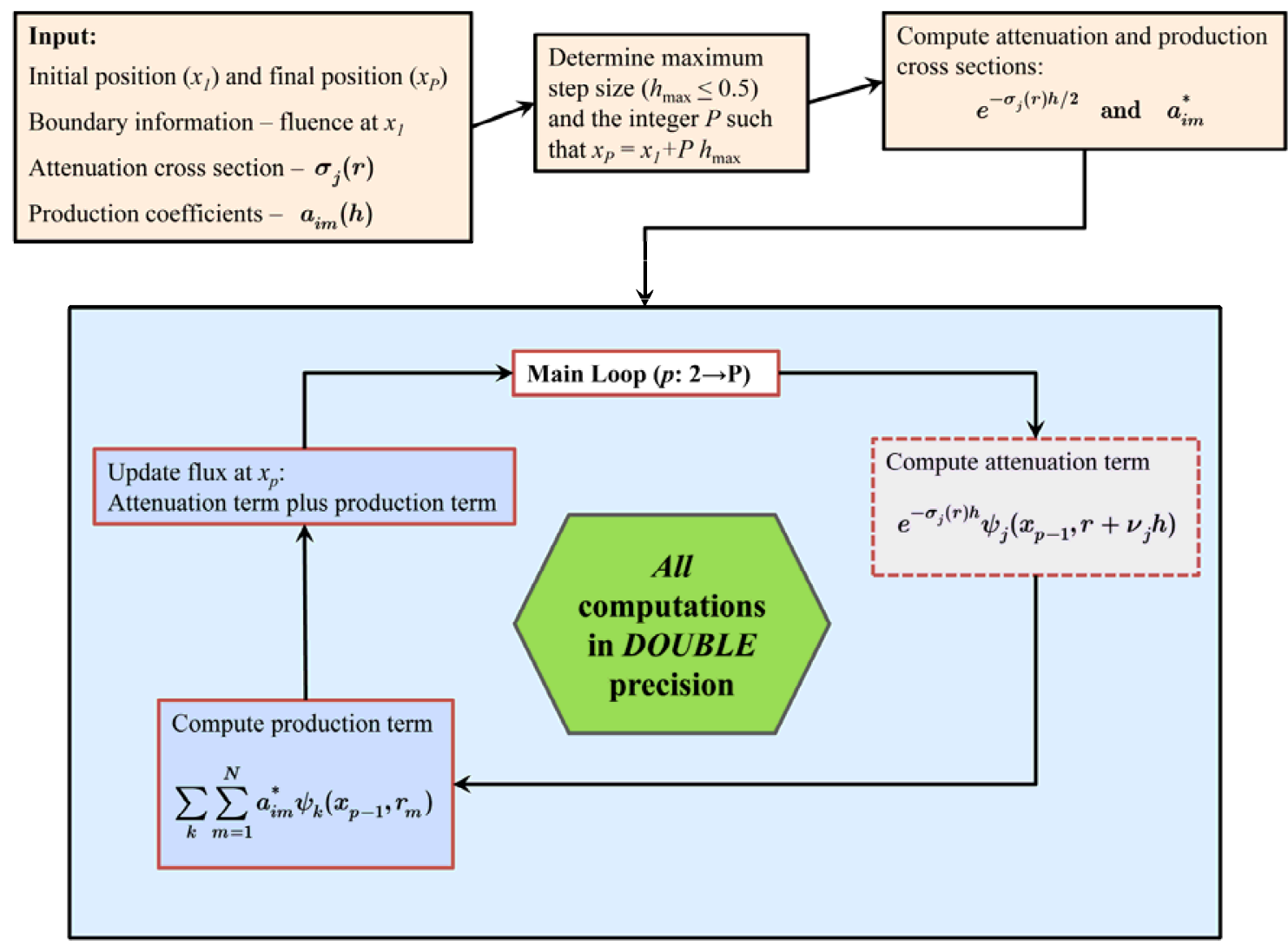

Figure 3. Process description of the new light particle transport algorithm.

Table 1. Number of interpolations per step in the old and new light particle transport algorithms.

\begin{tabular}{ccc}
\hline Grid-size & \multicolumn{2}{c}{ Interpolations } \\
\cline { 2 - 3 }$(N)$ & Old & New \\
\hline 100 & 364,800 & 600 \\
150 & 817,200 & 900 \\
300 & $3,254,400$ & 1800 \\
500 & $9,024,000$ & 3000 \\
\hline
\end{tabular}

\section{Heavy Ion Marching Equation Numerical Methods}

The heavy ion marching equation was given previously as

$$
\begin{gathered}
\psi_{j}(x+h, r)=e^{-\beta_{j}(r, h)} \psi_{j}\left(x, r+\nu_{j} h\right) \\
+\sum_{k>j} \frac{\nu_{j}}{\nu_{k}} \sigma_{j k}\left(r+\nu_{j} h / 2\right) \psi_{k}\left(x, r+\left(\nu_{j}+\nu_{k}\right) h / 2\right) \\
\quad \times \frac{e^{-\sigma_{j}\left(r+\nu_{j} h / 2\right) h}-e^{-\sigma_{k}\left(r+\left(\nu_{j}+\nu_{k}\right) h / 2\right) h}}{\sigma_{k}\left(r+\left(\nu_{j}+\nu_{k}\right) h / 2\right)-\sigma_{j}\left(r+\nu_{j} h / 2\right)} .
\end{gathered}
$$


Inspection of equation (95) reveals that the only numerical technique required is interpolation. The interpolation routine used in the heavy ion marching algorithm [Wilson et al. 1995] is the same routine used in the light particle marching algorithm. Thus, to improve efficiency and robustness, this routine has been replaced with the updated version mentioned in the previous section. A comparison of the routines will be given shortly.

\section{Coupling Light Particle and Heavy Ion Solutions for GCR}

The GCR environment is composed of energetic protons, alpha particles, and heavier ions with $Z>2$. In order to simultaneously transport all of these particles using the methods outlined above, the light particle and heavy ion marching algorithms must be coupled. This coupling has never been explicitly documented, but has existed in HZETRN for some time. The complete marching algorithm for GCR environments can be succinctly written as

$$
\begin{aligned}
\psi_{j}\left(x+h, r_{i}\right)= & e^{-\sigma_{j}\left(r_{i}\right) h} \psi_{j}\left(x, r_{i}+\nu_{j} h\right) \\
& +H(J-j) \sum_{k} \sum_{m=1}^{N} a_{i m}^{*} \psi_{k}\left(x, r_{m}\right) \\
& +\sum_{k>J^{*}} \frac{\nu_{j}}{\nu_{k}} \sigma_{j k}\left(r+\nu_{j} h / 2\right) \psi_{k}\left(x, r+\left(\nu_{j}+\nu_{k}\right) h / 2\right) \\
& \times \frac{e^{-\sigma_{j}\left(r+\nu_{j} h / 2\right) h}-e^{-\sigma_{k}\left(r+\left(\nu_{j}+\nu_{k}\right) h / 2\right) h}}{\sigma_{k}\left(r+\left(\nu_{j}+\nu_{k}\right) h / 2\right)-\sigma_{j}\left(r+\nu_{j} h / 2\right)}
\end{aligned}
$$

The index $J^{*}$ refers to the heaviest light ion $\left({ }^{4} \mathrm{He}\right)$, the Heaviside function is

$$
H(J-j) \equiv\left\{\begin{array}{ll}
0, & j>J \\
1, & j \leq J
\end{array},\right.
$$

and the lower limit of the last summation term is given by

$$
J^{*} \equiv\left\{\begin{array}{ll}
j, & j>J \\
J & , j \leq J
\end{array} .\right.
$$

For completeness, the same marching equation is given in terms of the old marching algorithms as

$$
\begin{aligned}
\psi_{j}\left(x+h, r_{i}\right)= & e^{-\sigma_{j}\left(r_{i}\right) h} \psi_{j}\left(x, r_{i}+\nu_{j} h\right) \\
& +H(J-j) \sum_{k} \frac{\bar{\nu}_{j}}{\bar{\nu}_{k}} \sum_{m=i}^{N-1} e^{-\sigma_{j}\left(r_{i}\right) h / 2-\sigma_{k}\left(r_{m}\right) h / 2} F_{j k}^{\Delta}\left(r_{i}, \bar{r}_{m}+\nu_{j} h / 2 ; h\right) \\
& \times\left[\Psi_{j}\left(x, r_{m}+\left(\nu_{j}+\nu_{k}\right) h / 2\right)-\Psi_{j}\left(x, r_{m+1}+\left(\nu_{j}+\nu_{k}\right) h / 2\right)\right] \\
& +\sum_{k>J^{*}} \frac{\nu_{j}}{\nu_{k}} \sigma_{j k}\left(r+\nu_{j} h / 2\right) \psi_{k}\left(x, r+\left(\nu_{j}+\nu_{k}\right) h / 2\right) \\
& \times \frac{e^{-\sigma_{j}\left(r+\nu_{j} h / 2\right) h}-e^{-\sigma_{k}\left(r+\left(\nu_{j}+\nu_{k}\right) h / 2\right) h}}{\sigma_{k}\left(r+\left(\nu_{j}+\nu_{k}\right) h / 2\right)-\sigma_{j}\left(r+\nu_{j} h / 2\right)}
\end{aligned}
$$

The only difference between equation (96) and (99) is the form of the light particle production term. The physical meaning of the coupling is the same in either equation.

The simplest way to explain and understand the GCR transport equation is by considering two separate cases. First, suppose we are interested in the transport of a single heavy ion with particle subscript 
$p$. Since $p$ refers to a heavy ion, it must be true that $p>J$, so that $H(J-p)=0$ and $J^{*}=p$. Therefore, the light particle production term is neglected and only the heavy ion transport equation remains

$$
\begin{aligned}
\psi_{p}\left(x+h, r_{i}\right)= & e^{-\sigma_{p}\left(r_{i}\right) h} \psi_{p}\left(x, r_{i}+\nu_{p} h\right) \\
& +\sum_{k>p} \frac{\nu_{p}}{\nu_{k}} \sigma_{p k}\left(r+\nu_{p} h / 2\right) \psi_{k}\left(x, r+\left(\nu_{p}+\nu_{k}\right) h / 2\right) \\
& \times \frac{e^{-\sigma_{p}\left(r+\nu_{p} h / 2\right) h}-e^{-\sigma_{k}\left(r+\left(\nu_{p}+\nu_{k}\right) h / 2\right) h}}{\sigma_{k}\left(r+\left(\nu_{p}+\nu_{k}\right) h / 2\right)-\sigma_{p}\left(r+\nu_{p} h / 2\right)}
\end{aligned}
$$

There is nothing surprising in this result; it simply reiterates that heavy ions are transported with the heavy ion transport equation.

Now, suppose that we are interested in the transport of a single light particle with particle subscript $p$. Since $p$ refers to a light particle, it must be true that $p \leq J$, so that $H(J-p)=1$ and $J^{*}=J$. In this case, the marching algorithm becomes

$$
\begin{aligned}
\psi_{p}\left(x+h, r_{i}\right)= & e^{-\sigma_{p}\left(r_{i}\right) h} \psi_{p}\left(x, r_{i}+\nu_{p} h\right) \\
& +\sum_{k} \sum_{m=1}^{N} a_{i m}^{*} \psi_{k}\left(x, r_{m}\right) \\
& +\sum_{k>J} \frac{\nu_{p}}{\nu_{k}} \sigma_{p k}\left(r+\nu_{p} h / 2\right) \psi_{k}\left(x, r+\left(\nu_{p}+\nu_{k}\right) h / 2\right) \\
& \quad \times \frac{e^{-\sigma_{p}\left(r+\nu_{p} h / 2\right) h}-e^{-\sigma_{k}\left(r+\left(\nu_{p}+\nu_{k}\right) h / 2\right) h}}{\sigma_{k}\left(r+\left(\nu_{p}+\nu_{k}\right) h / 2\right)-\sigma_{p}\left(r+\nu_{p} h / 2\right)}
\end{aligned}
$$

which is identical to the light particle marching algorithm, except that the additional summation term has been added. The second summation term is taken over all heavy ions and represents the source of light particle projectile fragments produced by collisions between heavy ions and the target media. This second summation term is the coupling mechanism between the light particle and heavy ion marching equations.

\section{Round-Off Error, Coding Mistakes, and Interpolation}

In this section, the impact of single precision round-off error, coding errors and interpolation on exposure quantities and overall code stability is examined. First, the errors caused by single precision calculations in the light particle cross sections are analyzed. These errors are significant enough to cause instabilities in the transport algorithms that are problematic for material thicknesses $>50 \mathrm{~g} / \mathrm{cm}^{2}$. Various coding errors related to light particle production cross sections that have a non-negligible impact on fluence spectra and exposure quantities are also discussed. Finally, the new interpolation routine mentioned in sections 6 and 7 is compared to the previous routine [Wilson et al. 1995], and the improved efficiency and robustness of the updated algorithm is shown. For the convergence tests (discussed later), all the errors, mistakes, and interpolation routines have been corrected in the original and updated algorithms so that any differences can be attributed only to the numerical algorithms.

Subtractive cancellation can occur in computational algorithms when the difference is computed between two numbers that are nearly equal in a given precision. This problem was occurring for some of the light particle cross section calculations. Recall that the integrated light particle production cross sections were computed as a difference of two cumulative production spectra according to

$$
F_{j k}^{\Delta}\left(r, r^{\prime} ; h\right) \equiv \frac{1}{\nu_{j}}\left[\int_{0}^{\varepsilon\left(r+\nu_{j} h\right)} \sigma_{j k}\left(E, E^{\prime}\right) d E-\int_{0}^{\varepsilon(r)} \sigma_{j k}\left(E, E^{\prime}\right) d E\right]
$$


For large fragment energies, where $\varepsilon\left(r+\nu_{j} h\right) \approx \varepsilon(r)$, the two integrals in equation (102) become nearly equal. Thus, in single precision, the difference will lose numerical precision and may be evaluated as zero. An example of this is shown in Figure 4, where the integrated light particle production cross section, $F_{j k}^{\Delta}\left(r, r^{\prime} ; h\right)$, for $1 \mathrm{AGeV}^{4} \mathrm{He}$ ions producing ${ }^{3} \mathrm{H}$ and ${ }^{3} \mathrm{He}$ in aluminum [Cucinotta et al. 1993] are evaluated in single and double precision. For fragment energies larger than $\sim 50 \mathrm{AMeV}$, the single precision results are zero and are therefore not visible on the plot. Similar results were also found for other reactions and projectile energies. The impact of this round-off error on particle fluence spectra can be seen in Figure 5, where the secondary ${ }^{3} \mathrm{H}$ and ${ }^{3} \mathrm{He}$ fluences at $100 \mathrm{~g} / \mathrm{cm}^{2}$ in aluminum exposed to the ${ }^{4} \mathrm{He}$ component of the 1977 solar minimum GCR spectrum [O'Neill and Badhwar 2006] are shown. The instabilities in the single precision results above $10 \mathrm{AGeV}$ are clear. As these results are propagated to larger depths, the instabilities grow in magnitude, reach lower energies, and eventually cause algorithm failure.

Various coding errors were also discovered in the algorithms that generate the light particle production cross sections. To understand these coding errors, it is necessary to first review how differential production cross sections are represented in HZETRN. From Wilson et al. [1991], the light particle differential cross sections can be expressed as

$$
\sigma_{j k}\left(E, E^{\prime}\right)=m_{j k}\left(E^{\prime}\right) \sigma_{k}\left(E^{\prime}\right) f_{j k}\left(E, E^{\prime}\right)
$$

where $\sigma_{k}\left(E^{\prime}\right)$ is the macroscopic cross section for the projectile, $m_{j k}\left(E^{\prime}\right)$ is the mean multiplicity of type $j$ particles produced by type $k$ particles with energy $E$, and $f_{j k}\left(E, E^{\prime}\right)$ is the energy spectrum of the reaction. The spectral term is normalized so that

$$
\int_{0}^{\infty} f_{j k}\left(E, E^{\prime}\right) d E=1
$$

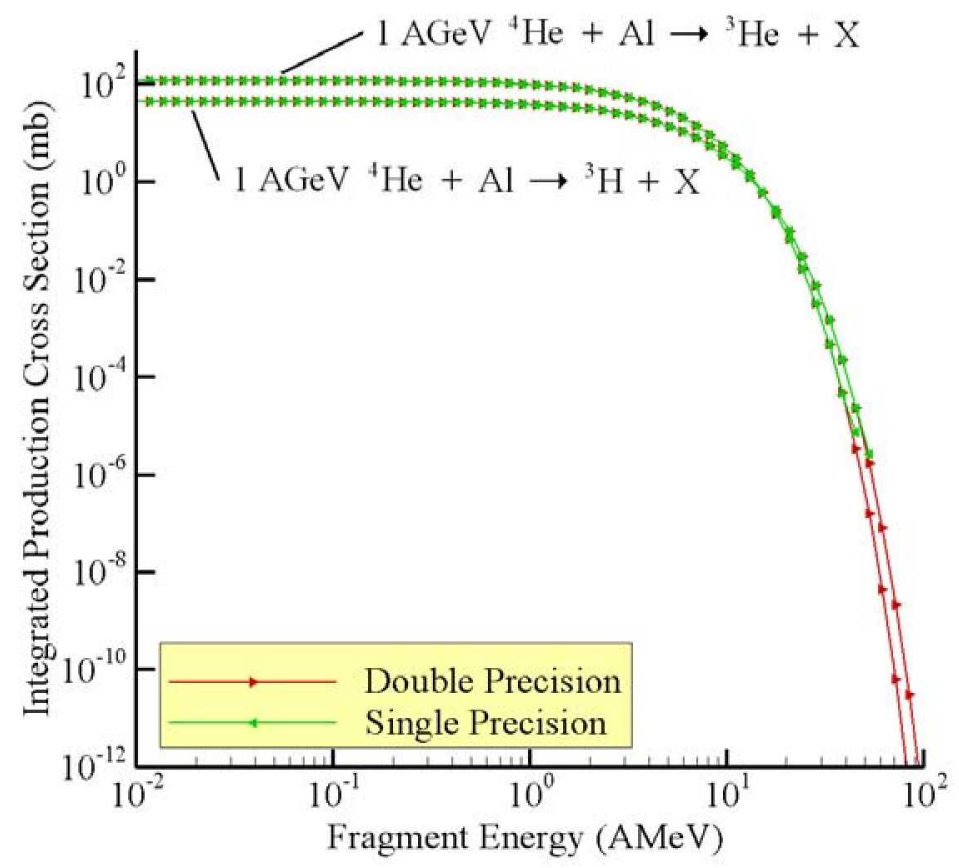

Figure 4. Integrated production cross section for a $1 \mathrm{AGeV}{ }^{4} \mathrm{He}$ ion producing ${ }^{3} \mathrm{He}$ and ${ }^{3} \mathrm{H}$ in aluminum. 

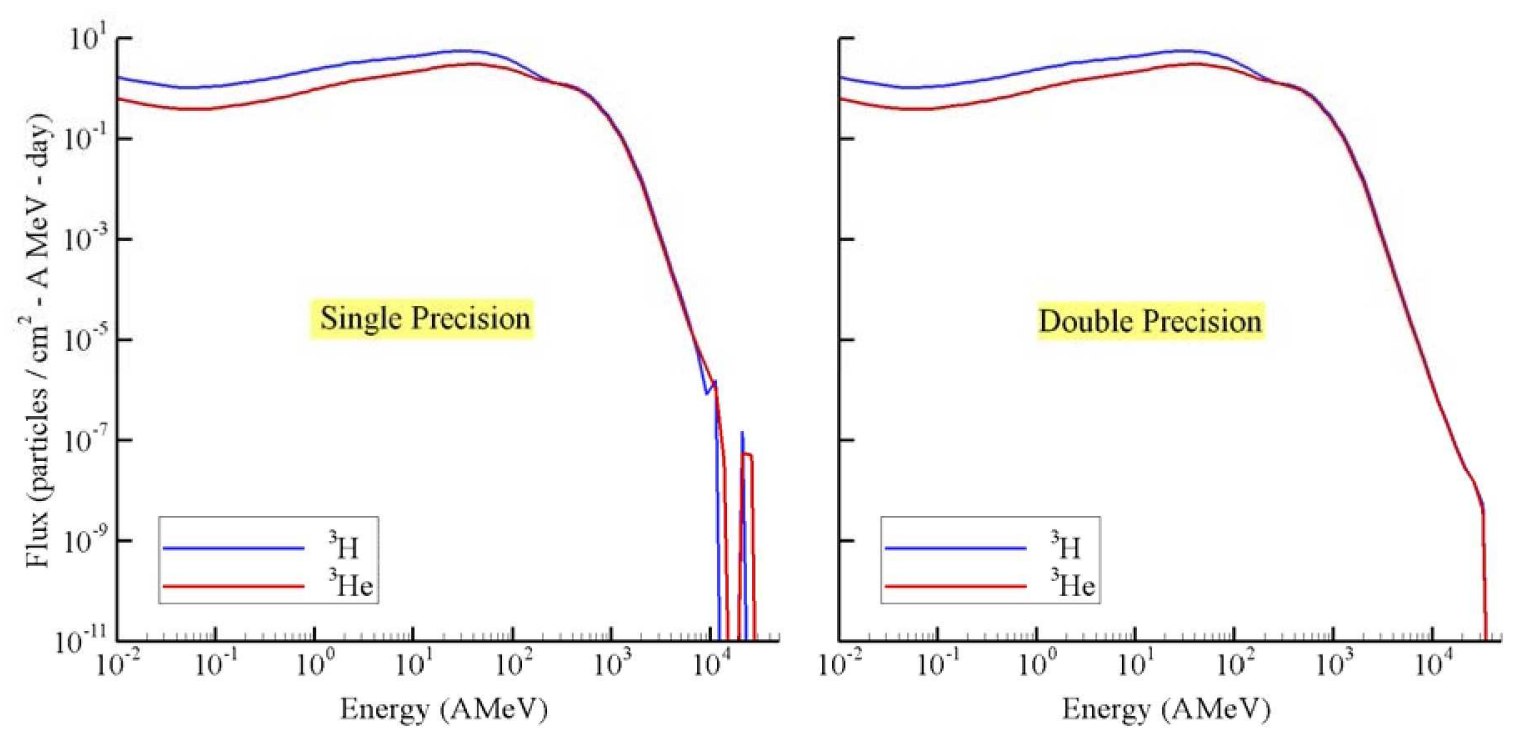

Figure 5. Secondary ${ }^{3} \mathrm{H}$ and ${ }^{3} \mathrm{He}$ fluences computed using single and double precision cross sections at 100 $\mathrm{g} / \mathrm{cm}^{2}$ in aluminum exposed to the ${ }^{4} \mathrm{He}$ component of the 1977 solar minimum GCR spectrum.

For each of the reactions, there is a maximum fragment energy, $E_{m}$, above which the spectral term is identically zero. For example, for nucleon production from nucleon-nucleus interactions, the fragment energy is required to be less than the projectile energy; thus, $E_{m f}=E^{\prime}$ [Wilson et al. 1991]. These constraints were imposed on the integrated light particle production cross sections in equation (102) by setting $F_{j k}^{\Delta}\left(r, r^{\prime} ; h\right)=0$ if either $\varepsilon\left(r+\nu_{j} h\right)$ or $\varepsilon(r)$ were greater than $E_{m f}$. This constraint correctly accounts for the case when both energies are greater than $E_{m f}$, but it does not account for the case when $\varepsilon\left(r+\nu_{j} h\right)>$ $E_{m f}$ and $\varepsilon(r)<E_{m f}$. One would expect this error to cause a small under-prediction of secondary particles by effectively truncating the integration limits in equation (102). The necessary corrections have been made so that the integrated light particle production cross sections are now properly evaluated up to their limits for all energies and step-sizes. The effect of this algorithmic error is shown in Figure 6 where we have computed the proton fluences at $100 \mathrm{~g} / \mathrm{cm}^{2}$ in aluminum exposed to the August 1972 King SPE [King 1974]. As expected, the results with the maximum fragment energy coding error, " $E_{m f}$ Error," under-predict the corrected results, " $E_{m f}$ Corrected." The numerical errors shown in Figure 6 are on the order of 25\% below $1 \mathrm{MeV}$.

Another coding error was found in which a modified proton elastic cross section was incorrectly added into a non-elastic reaction channel. For the reaction, $n+T \rightarrow p+X$, where $n$ is a neutron, $T$ is the initial target state, $p$ is a proton, and $X$ is the final target state, the production cross section was evaluated as

$$
\sigma_{p n}\left(E, E^{\prime}\right)=\left[m_{p n}\left(E^{\prime}\right) \sigma_{n}\left(E^{\prime}\right) f_{p n}\left(E, E^{\prime}\right)\right]+\sigma_{n}^{e l}\left(E^{\prime}\right) f_{p p}^{e l}\left(E, E^{\prime}\right)
$$

where the superscript "el" refers to elastic. The bracketed term contains the correct quantities for the reaction, and the non-bracketed term is the modified proton elastic cross section that should not be included. Notice that the additional term uses the neutron elastic cross section and the proton elastic spectrum. Thus, not only is the term used incorrectly, it is being evaluated improperly as well. Surprisingly, this error, coupled to the $E_{m f}$ error mentioned above, can produce results very near the corrected code. In Figures 7 and 8, the isolated and coupled effects of both errors are shown. In the Figures, " $E_{m f}$ Error" refers to the maximum fragment energy error mentioned previously, " $p^{e l}$ Error" refers to the proton elastic error in equation (105), and the "Corrected" results were obtained with both errors removed. The coding errors have very different impacts in aluminum and water. In the aluminum target, the proton elastic error has almost no effect when the maximum fragment energy error is present. However, in the water target, the proton elastic error has a large effect whether the maximum fragment energy error is present or not. 


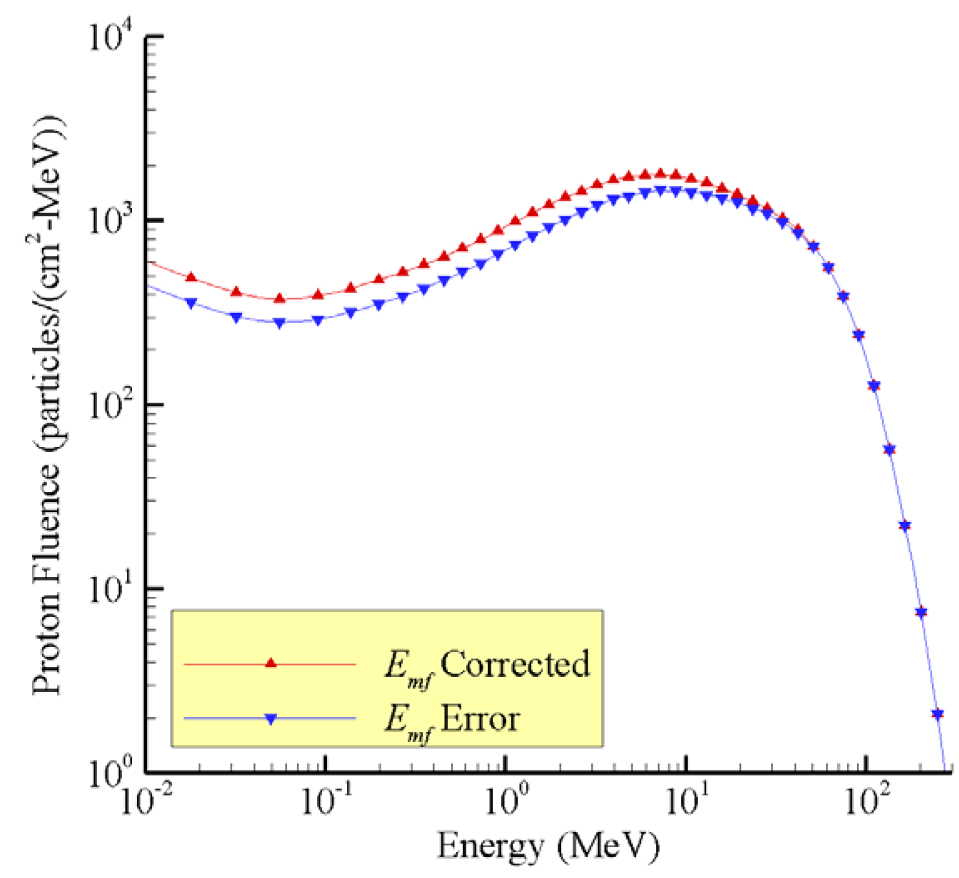

Figure 6. Proton fluences computed with and without the maximum fragment energy coding error at 100 $\mathrm{g} / \mathrm{cm}^{2}$ in aluminum exposed to the August 1972 King SPE.

A final coding error was found in the evaluation of direct knockout target fragments with mass $2 \leq$ $A \leq 4$. The spectral term for this reaction was parameterized as [Cucinotta et al. 1996]

$$
f_{j k}\left(E, E^{\prime}\right)=\frac{A_{j} \exp \left(-E A_{j} / w_{0}\right)}{w_{0}\left[1-\exp \left(-E^{\prime} / w_{0}\right)\right]},
$$

where $A_{j}$ is the fragment mass and $w_{0}$ is a spectral width dependent on the projectile energy given by

$$
w_{0}\left(E^{\prime}\right) \equiv\left\{\begin{array}{cl}
40+E^{\prime} / 5 & , \text { if } E^{\prime} \leq 800 \\
200 & , \text { if } E^{\prime}>800
\end{array} .\right.
$$

After reviewing the algorithm where this parameterization is found, it was determined that the fragment energy, $E$, instead of the projectile energy, $E$, was being incorrectly used in the evaluation of $w_{0}$. The effect of this spectral width coding error on secondary particle production is shown in Figure 9 where we have shown the ${ }^{4} \mathrm{He}$ and secondary ${ }^{2} \mathrm{H}$ flux spectra in $100 \mathrm{~g} / \mathrm{cm}^{2}$ of aluminum exposed to the 1977 solar minimum GCR environment. The dashed curves marked with " $w_{0}$ Error" are the results generated with the fragment energy in place of the projectile energy in the evaluation of $w_{0}$ in equations (106) and (107). The impact is significant between $10 \mathrm{AMeV}$ and $1000 \mathrm{AMeV}$. Similar results were also found in the secondary ${ }^{3} \mathrm{H}$ and ${ }^{3} \mathrm{He}$ spectra as well. 


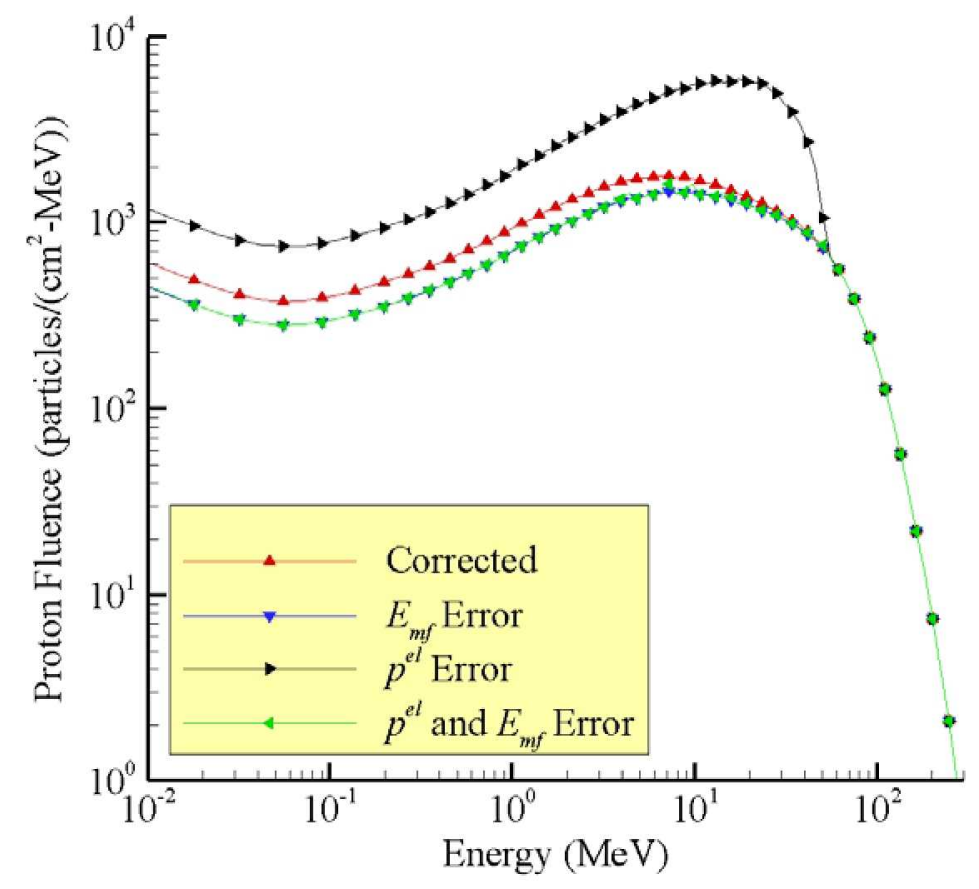

Figure 7. Proton fluences computed with and without the maximum fragment energy and proton elastic coding errors at $100 \mathrm{~g} / \mathrm{cm}^{2}$ in aluminum exposed to the August 1972 King SPE.

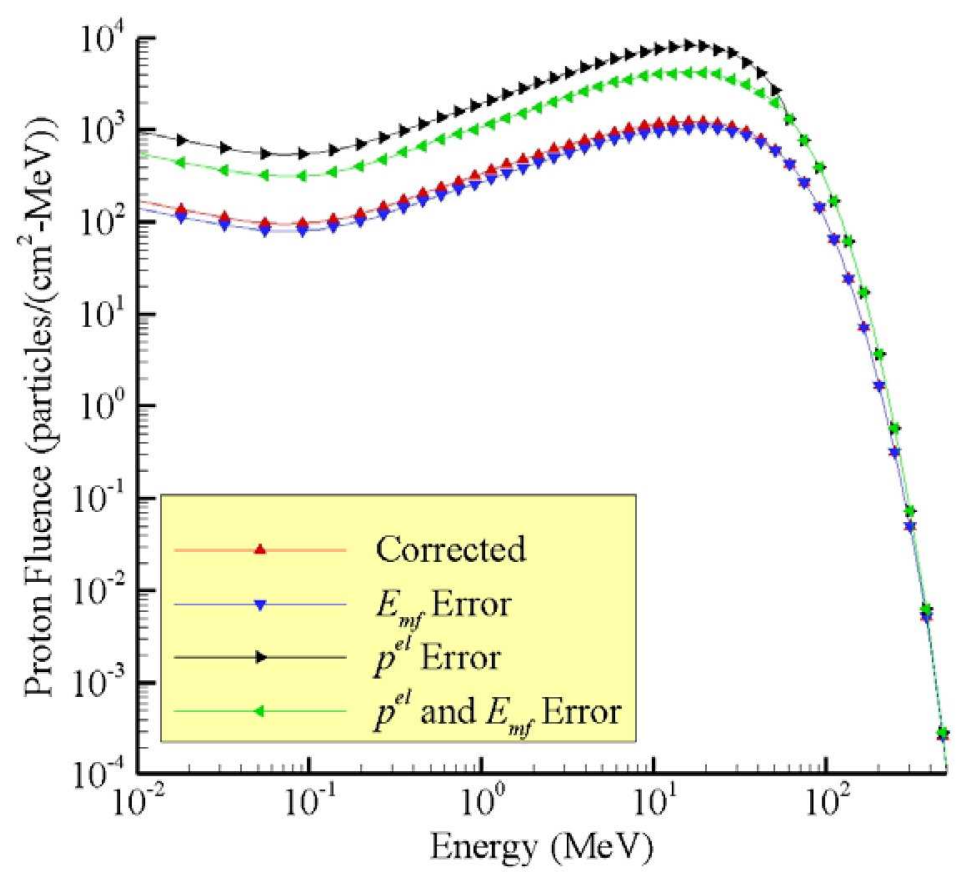

Figure 8. Proton fluences computed with and without the maximum fragment energy and proton elastic coding errors at $100 \mathrm{~g} / \mathrm{cm}^{2}$ in water exposed to the August $1972 \mathrm{King}$ SPE. 


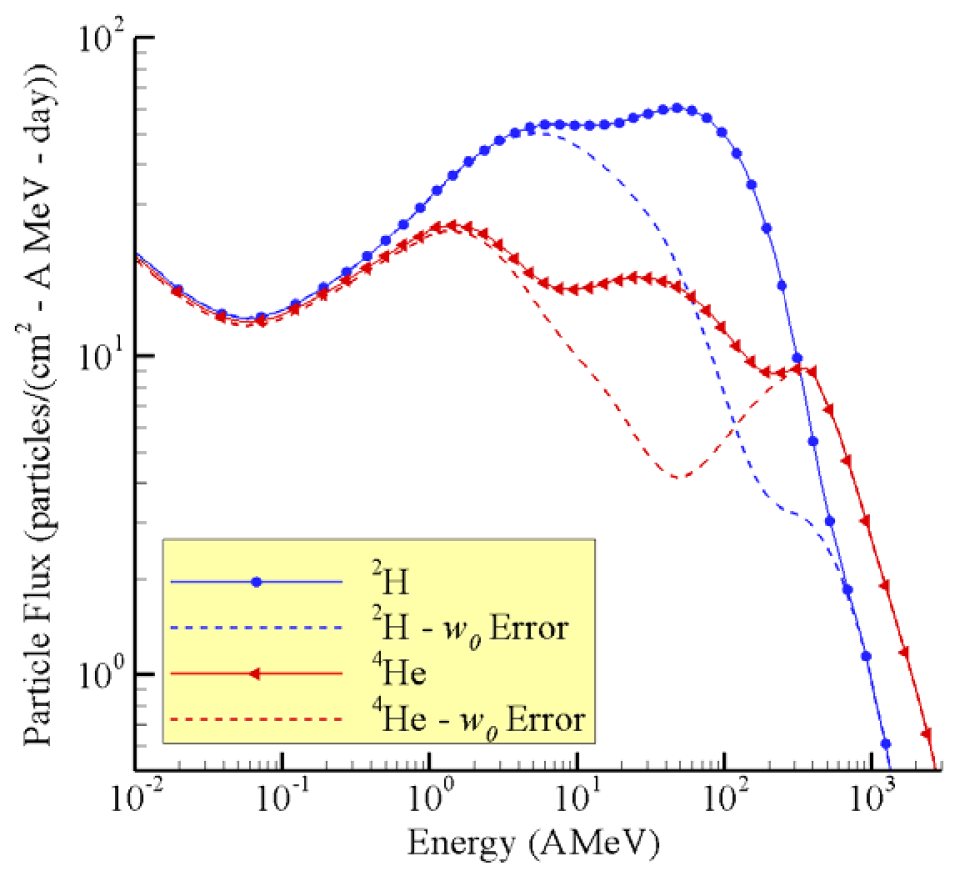

Figure 9. ${ }^{4} \mathrm{He}$ and secondary ${ }^{2} \mathrm{H}$ flux spectra computed with and without the spectral width error in 100 $\mathrm{g} / \mathrm{cm}^{2}$ of aluminum exposed to the 1977 solar minimum GCR environment.

Lastly, the interpolation routine used in previous versions of HZETRN is compared to a new interpolation routine that is faster and more robust. Both routines used cubic Lagrange interpolating polynomials. A cubic Lagrange interpolating polynomial requires four data points $\left(x_{i}, y_{i}\right),\left(x_{i+1}, y_{i+1}\right),\left(x_{i+2}\right.$, $\left.y_{i+2}\right)$, and $\left(x_{i+3}, y_{i+3}\right)$. A common choice is to choose the four abscissa points symmetrically about the interpolating point so that $x_{i+1} \leq x \leq x_{i+2}$. However, if the interpolating point is near the boundary of the domain, one may also choose $x_{\mathrm{i}} \leq x \leq x_{\mathrm{i}+1}$ or $x_{\mathrm{i}+2} \leq x \leq x_{\mathrm{i}+3}$. The log-log cubic Lagrange interpolating polynomial is obtained by taking the natural $\log$ of the four data points, $\left(x_{i}, y_{i}\right),\left(x_{i+1}, y_{i+1}\right),\left(x_{i+2}, y_{i+2}\right),\left(x_{i+3}\right.$, $\left.y_{i+3}\right)$, and the interpolation point, $x$. The interpolated value is then exponentiated to account for the initial logarithm.

The interpolation routine previously used in the transport algorithms [Wilson et al. 1995] performed $\log -\log$ cubic interpolation and extrapolation. The search procedure used to find the abscissa points closest to the interpolation point was linear and searched in one direction from the origin (started from the first data point and searched forward one point at a time). All logarithms and exponentials were computed within the algorithm. No logic was included to ensure that extrapolated results matched the trends of the data within the domain (ie. increasing or decreasing). Also, no logic was included to check for non-smooth data.

In the updated algorithm, the search procedure used to find the abscissa points is linear and searches in either direction from the previously used interpolation point. Thus, when the routine is called repeatedly with an ordered or sequential set of interpolation points, the search algorithm uses previous results to find the nearest abscissa points. The new algorithm also avoids the use of logarithms and exponentials. Instead, the natural log of the data points is computed once prior to interpolation and used repeatedly. The extrapolation procedure has been switched to $\log -\log$ linear. This will ensure that extrapolated results always match the trend of the data near the end of the domain. Logic has also been included to check for non-smooth data. In the event that the interpolated result does not lay between its nearest two data points, the interpolated value is re-computed using linear interpolation. The benefit of this logic for non-smooth data sets will be examined shortly. 
To show the improved efficiency of the updated algorithm, the August 1972 King SPE is transported through $100 \mathrm{~g} / \mathrm{cm}^{2}$ of aluminum using a step-size of $h=0.5 \mathrm{~g} / \mathrm{cm}^{2}$ and neglect all nuclear production. The transport solution is given simply as

$$
\psi_{p}(x+h, r)=e^{-\sigma_{p}(r) h} \psi_{p}(x, r+h),
$$

requiring only interpolation to compute $\psi_{p}(x, r+h)$. It took $\sim 4.9$ milliseconds to reach $100 \mathrm{~g} / \mathrm{cm}^{2}$ using the new interpolation routine, and it took $\sim 16.3$ milliseconds to reach $100 \mathrm{~g} / \mathrm{cm}^{2}$ using the old interpolation routine. The new routine is almost 3.5 times faster than the previous one.

To show the improved robustness of the updated algorithm, we propagate a modified form of the August 1972 King SPE through $2 \mathrm{~g} / \mathrm{cm}^{2}$ of aluminum using a step-size of $h=0.5 \mathrm{~g} / \mathrm{cm}^{2}$. The modified spectrum has been adjusted so that the proton fluence above $50 \mathrm{MeV}$ is 1.0 particles $/\left(\mathrm{cm}^{2}-\mathrm{MeV}\right)$. The results are shown in Figure 10. Note that the original discontinuity at $50 \mathrm{MeV}$ has been shifted to approximately $20 \mathrm{MeV}$ because of atomic interactions in the target. The benefit of the added logic for nonsmooth data is clear. The old algorithm over-predicts the results below $20 \mathrm{MeV}$, especially near the discontinuity; it also sharply under-predicts the results just past the discontinuity. This oscillatory behavior is characteristic of cubic polynomials and can be problematic when such errors are propagated to larger depths. Conversely, the new algorithm properly interpolates through a discontinuity and introduces no oscillatory behavior.

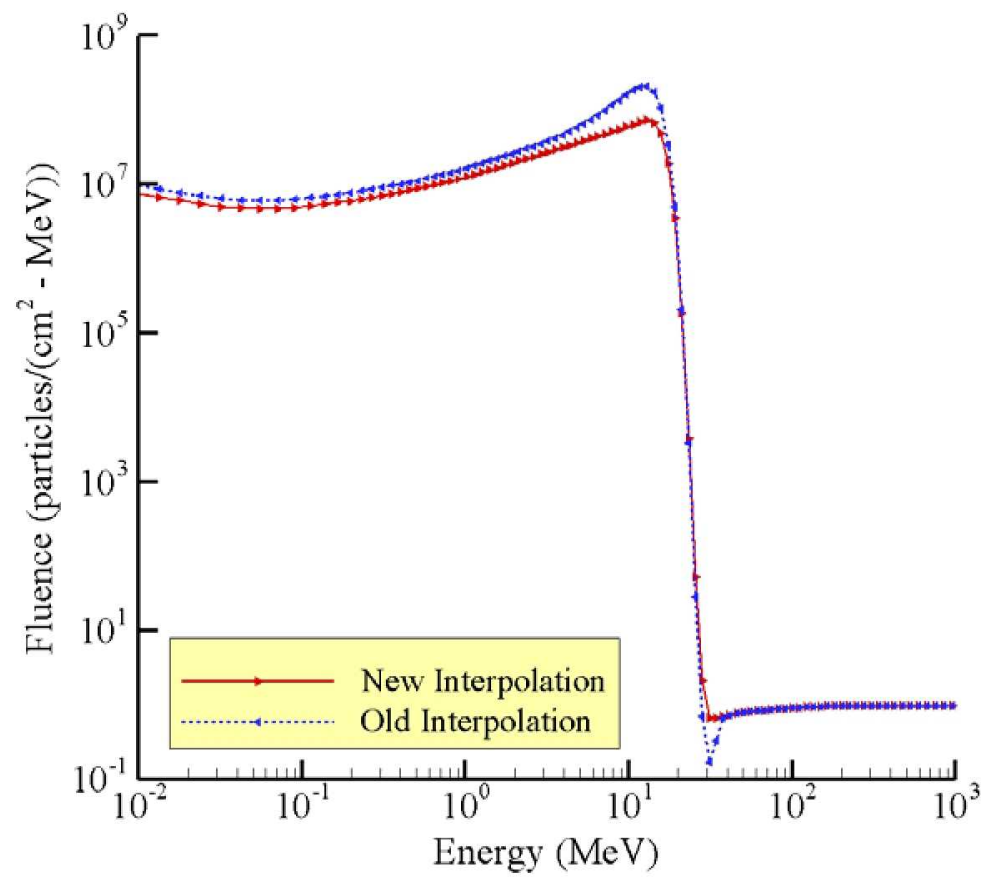

Figure 10. Comparison of old and new interpolation routines in $2 \mathrm{~g} / \mathrm{cm}^{2}$ of aluminum exposed to a modified form of the August 1972 King SPE. 
To show the combined effects of the errors identified in this section, we compare dose values computed with the original code (single precision, $E_{m f}$ error, $p^{e l}$ error, $w_{0}$ error, and old interpolation) and corrected code at $100 \mathrm{~g} / \mathrm{cm}^{2}$ of aluminum and water exposed to the August $1972 \mathrm{King}$ SPE and 1977 solar minimum GCR environments. The data are given in Table 2. First, note that the SPE data in aluminum and GCR data in aluminum and water are surprisingly similar despite the individual effects of the errors shown above. This would indicate that many of the errors are competing (ie. cancelling effects) and shows how difficult these errors can be to find and correct. The large differences in the water target exposed to the SPE are caused almost entirely by the $p^{e l}$ error. The $w_{0}$ error causes the code with errors to under-predict doses in aluminum. The results in the water target exposed to the GCR show the combined effects of two errors. While the $p^{e l}$ error would cause an over-prediction, the $w_{0}$ error causes an under-prediction, and the combined effect is a result very near the corrected code. It should be noted that for different materials or environments, the results are unpredictable.

Table 2. Dose at $100 \mathrm{~g} / \mathrm{cm}^{2}$ in aluminum and water exposed to August $1972 \mathrm{King}$ SPE and 1977 solar minimum GCR. The units for the SPE results are cGy, and the units for the GCR results are cGy/day.

\begin{tabular}{lcccc}
\hline & \multicolumn{2}{c}{ SPE } & \multicolumn{2}{c}{ GCR } \\
\cline { 2 - 5 } & Aluminum & Water & Aluminum & Water \\
\hline Corrected code & $3.28 \times 10^{-1}$ & $6.18 \times 10^{-2}$ & $4.72 \times 10^{-2}$ & $3.78 \times 10^{-2}$ \\
Code with Errors & $3.21 \times 10^{-1}$ & $1.27 \times 10^{-1}$ & $4.22 \times 10^{-2}$ & $3.77 \times 10^{-2}$ \\
\hline
\end{tabular}

\section{Convergence Study for Light Particle Transport in SPE Environments}

In this section, the total discretization error associated with the old and new light particle transport algorithms in HZETRN is examined by conducting a detailed convergence analysis in both step-size $(h)$ and energy grid-size $(N)$. For all comparisons, the round-off errors, coding mistakes, and interpolation routines mentioned in the previous section have been fixed in the original algorithms. This will allow a direct comparison between the original and updated numerical algorithms. The convergence analysis is completed by transporting the August 1972 King SPE spectrum through $100 \mathrm{~g} / \mathrm{cm}^{2}$ of aluminum and water. Six different energy grids were used with minimum and maximum energy values of $E_{\min }=0.01 \mathrm{AMeV}$ and $E_{\max }=2500 \mathrm{AMeV}$. The grids were equally $\log$ spaced in energy and contained $100,149,223,335,502$, and 753 points; these grids will be referred to as $E-100, E-149, E-223, E-335, E-502$, and $E-753$. Each grid represents a refinement of 1.5 in the grid spacing parameter,

$$
\Delta_{E}=\frac{\log \left(E_{\max }\right)-\log \left(E_{\min }\right)}{N-1} .
$$

Thus, for $N=100, \Delta_{E}=0.054525$, and for $N=149, \Delta_{E}=0.036473$. Along with these different energy grids, the following step-sizes (in $\mathrm{g} / \mathrm{cm}^{2}$ ) were used to propagate the boundary condition into the slabs: $h=2^{-1}$, $2^{-2}, 2^{-3}, \ldots, 2^{-11}$. Each step-size is a refinement of two in $h$. These eleven step-sizes, six energy grids, two materials, and two transport algorithms, were used to obtain fluence, dose, and dose equivalent values at various depths in the target media. These data are used to show that both algorithms converge as a function of step-size and energy grid-size for SPE boundary conditions. It is also determined that the new algorithm is more accurate than its predecessor. Finally, total discretization error estimates are given for the discretization parameters $\left(h=0.5 \mathrm{~g} / \mathrm{cm}^{2}\right.$ with 100 energy grid points) commonly used in HZETRN. The errors are expressed as percent difference from a highly accurate, or converged, numerical solution.

In Figures 11 and 12, dose equivalent as a function of depth in aluminum and water is given for both of the algorithms and all of the discretization parameters. The plot legends have been removed because there are 132 different curves (6 energy grids, 11 step-sizes, 2 algorithms), many of which are overlapping. The spread in the data at $100 \mathrm{~g} / \mathrm{cm}^{2}$ in aluminum is $\sim 3 \mathrm{cSv}(\sim 39 \%)$, and the spread in the data at $100 \mathrm{~g} / \mathrm{cm}^{2}$ in water is $-0.2 \mathrm{cSv}(\sim 26 \%)$. It is clear in these plots that both methods, with the various discretization parameters, produce similar results and that the errors appear to be slowly increasing as a 
function of depth in the material. Slightly larger errors are also found in aluminum. These errors will be discussed in detail later.

In Figures 13 and 14, the dose equivalent at $100 \mathrm{~g} / \mathrm{cm}^{2}$ in aluminum and water is plotted as a function of the energy grid-size, $N$, for three step-sizes. The three step-sizes were chosen to reduce data overlap and improve plot clarity. In Figures 15 and 16 , the dose equivalent at $100 \mathrm{~g} / \mathrm{cm}^{2}$ in aluminum and water is plotted as a function of the step-size, $h$, for all of the energy grids. Figures 13-16 show that both of the algorithms reach an asymptotic solution for step-sizes less than $0.01 \mathrm{~g} / \mathrm{cm}^{2}$ and energy grids with greater than 300 points. The use of step-sizes larger than $0.01 \mathrm{~g} / \mathrm{cm}^{2}$ results in a systematic under-prediction of dose equivalent regardless of the method or energy grid used. It is also clear that if larger energy grids and smaller step-sizes were considered, the methods would generate almost identical solutions. The percent difference between the dose equivalent values generated by the old and new algorithms with the finest discretization parameters $\left(h=2^{-11}, N=753\right)$ is $0.4 \%$ at $100 \mathrm{~g} / \mathrm{cm}^{2}$ in aluminum and $0.2 \%$ at $100 \mathrm{~g} / \mathrm{cm}^{2}$ in water. Thus, it is concluded that both methods converge to the same value as a function of step-size and energy grids size to the same solution for this SPE in aluminum and water.

Figures 15-16 indicate that there is little difference in spatial discretization error between the old and new algorithms. This is expected since the only difference between the algorithms is in the calculation of the source integral over energy. The new method appears to have a smaller energy discretization error in aluminum. This can be quantified by computing the percent difference between the $E-100$ and $E-753$ dose equivalent results from both algorithms with a step-size of $h=2^{-11} \mathrm{~g} / \mathrm{cm}^{2}$ at $100 \mathrm{~g} / \mathrm{cm}^{2}$. The percent difference between the $E-100$ and $E-753$ results for the old algorithm is $10.7 \%$ in aluminum and $2.4 \%$ in water, while the percent difference between the $E-100$ and $E-753$ results for the new algorithm is $2.5 \%$ in aluminum and $2.6 \%$ in water. The improvement in aluminum was achieved primarily because the new algorithm more accurately handles the sharply peaked production cross sections in aluminum, especially the neutron elastic cross sections that are important at large depths. The negligible accuracy loss in water shows that the methods are comparable when the production cross sections are relatively smooth, as is the case when hydrogen is present.

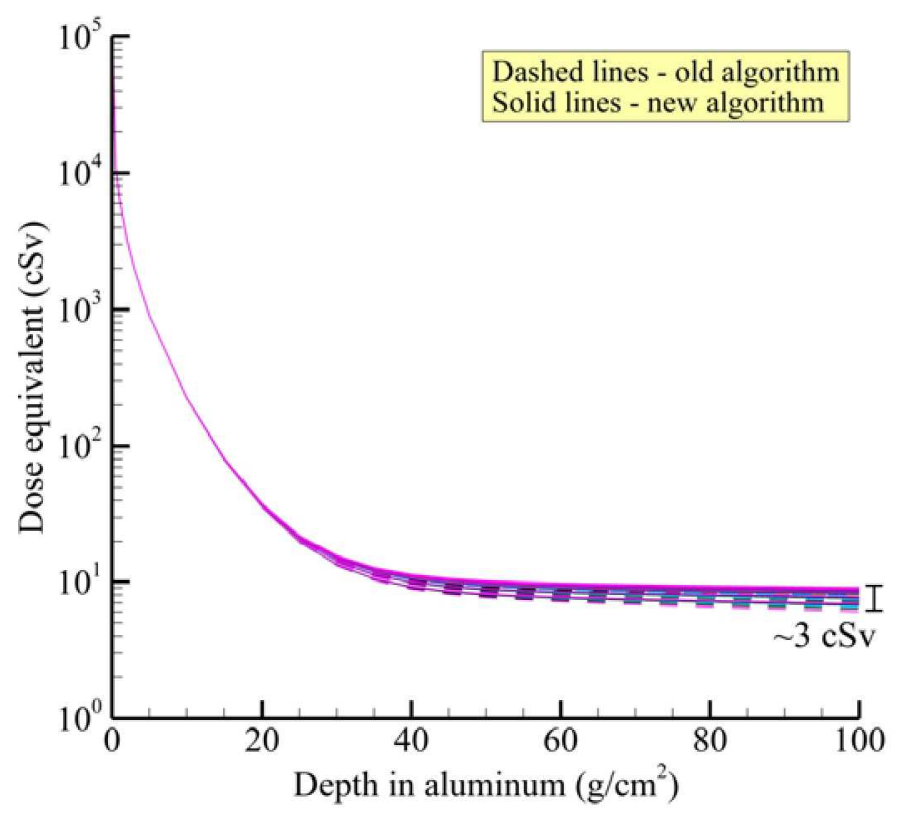

Figure 11. Dose equivalent versus depth in aluminum exposed to the August 1972 King SPE computed with both transport algorithms and all discretization parameters. 


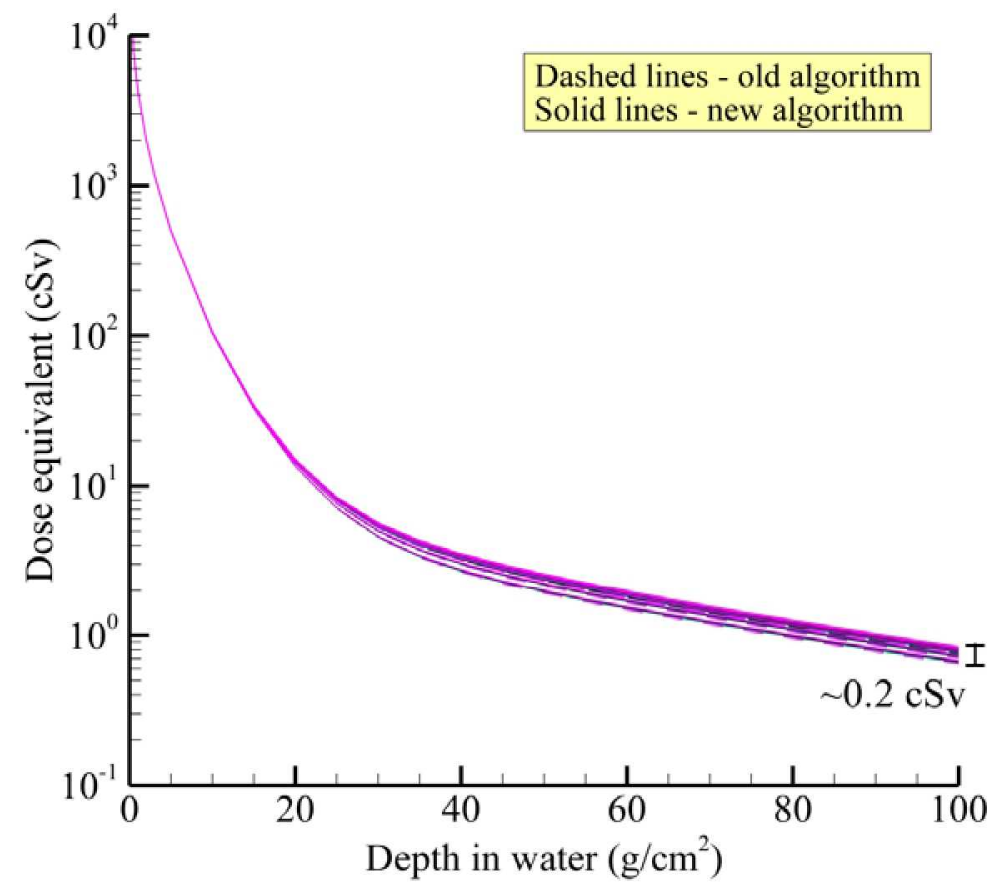

Figure 12. Dose equivalent versus depth in water exposed to the August 1972 King SPE computed with both transport algorithms and all discretization parameters.

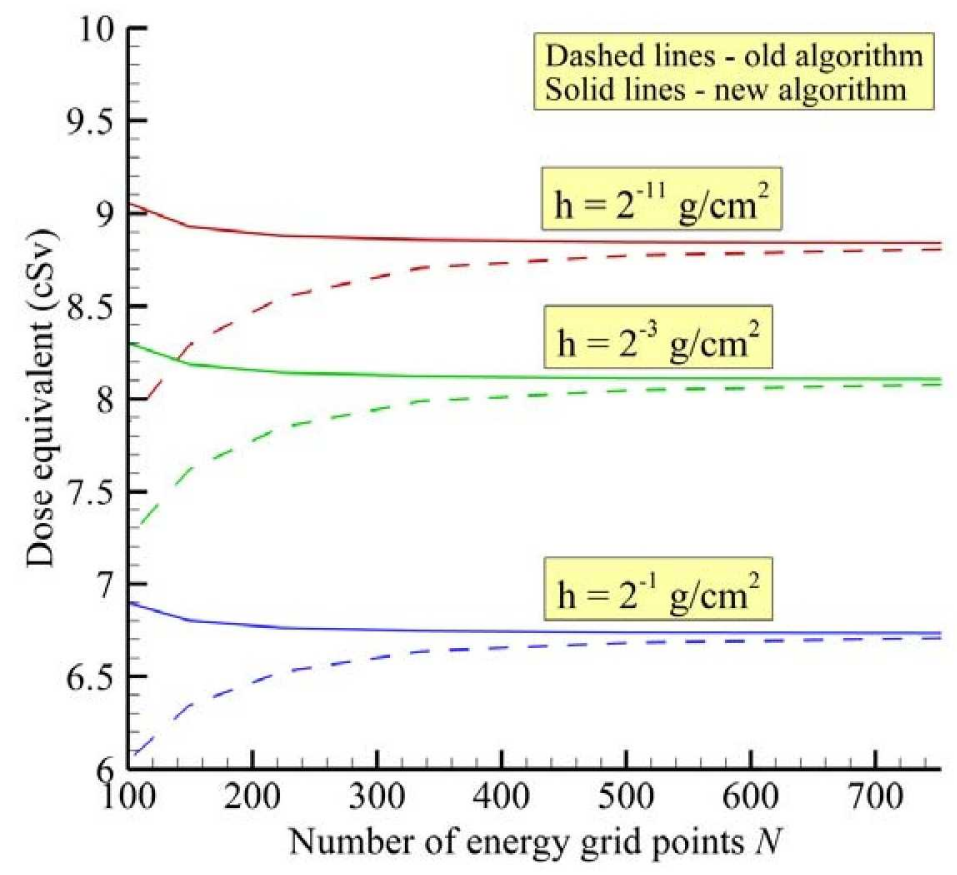

Figure 13. Dose equivalent versus energy grid-size at $100 \mathrm{~g} / \mathrm{cm}^{2}$ in aluminum exposed to the August 1972 King SPE. Both transport algorithms were used with three different step-sizes. 


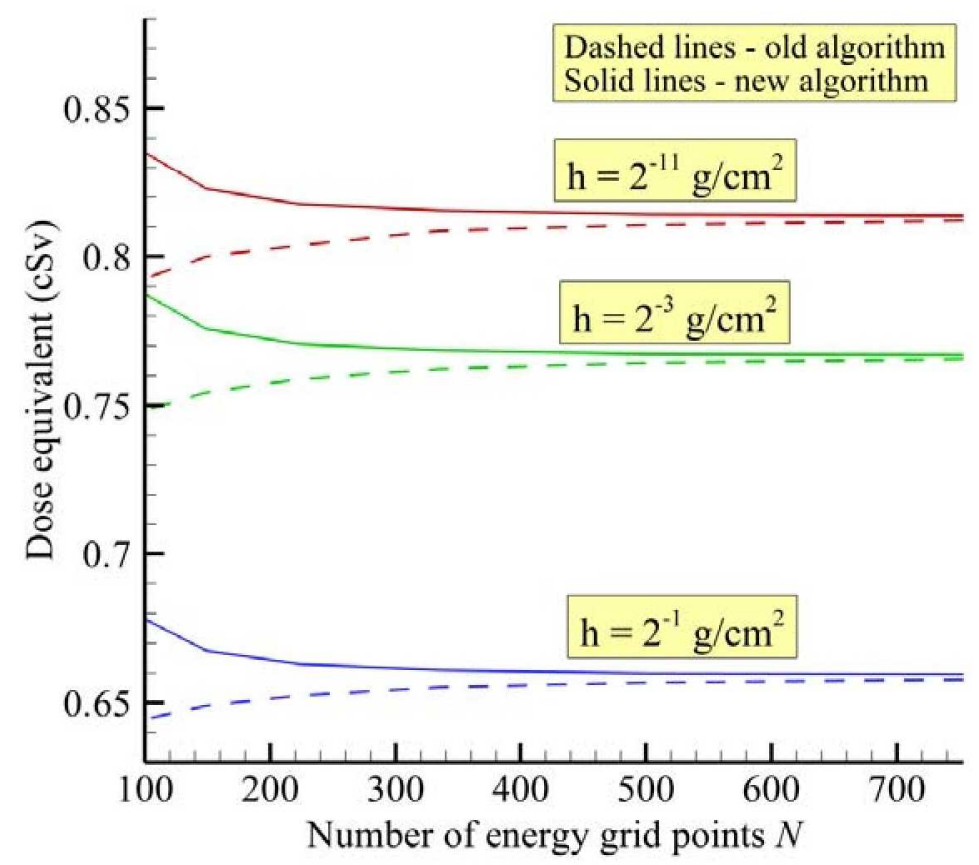

Figure 14. Dose equivalent versus energy grid-size at $100 \mathrm{~g} / \mathrm{cm}^{2}$ in water exposed to the August $1972 \mathrm{King}$ SPE. Both transport algorithms were used with three different step-sizes.

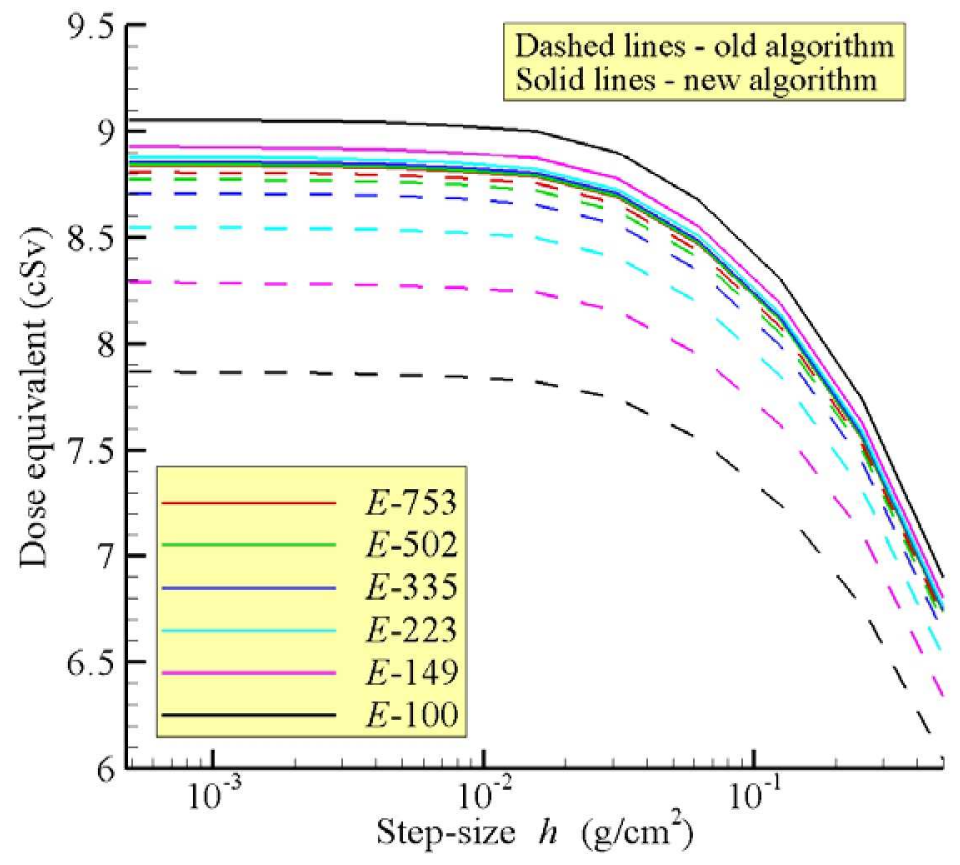

Figure 15. Dose equivalent versus step-size at $100 \mathrm{~g} / \mathrm{cm}^{2}$ in aluminum exposed to the August $1972 \mathrm{King}$ SPE. Both transport algorithms were used with six different energy grids. 


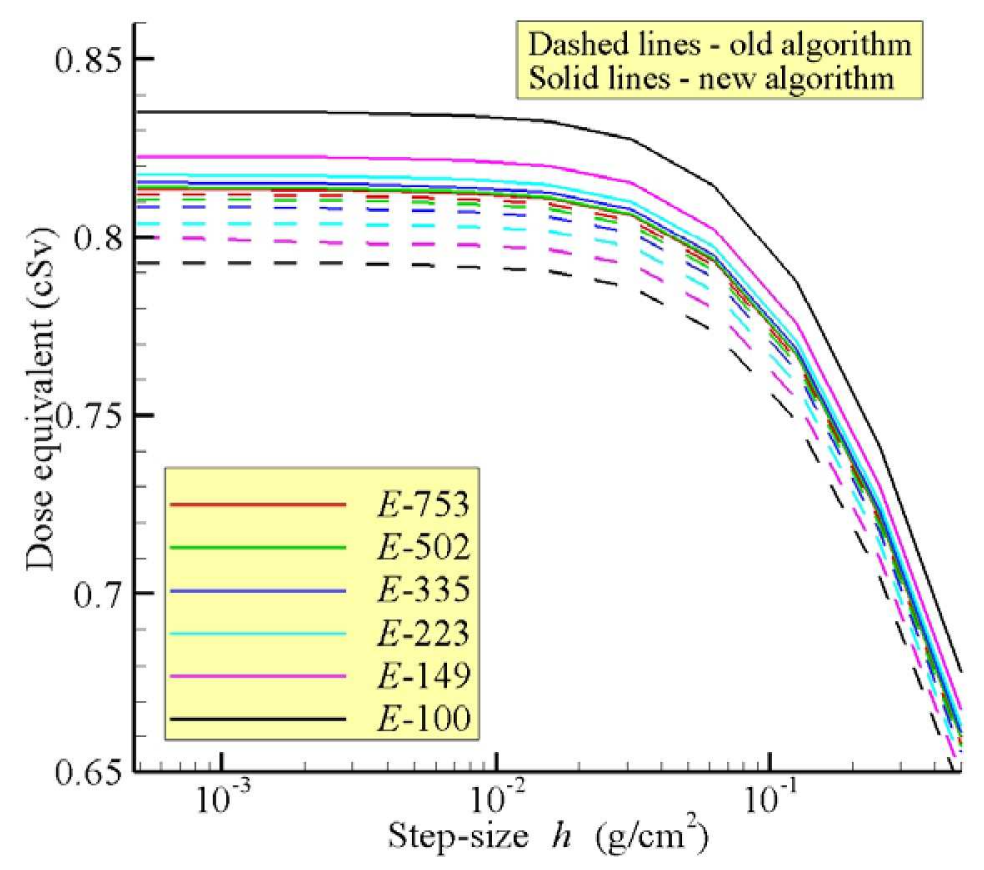

Figure 16. Dose equivalent versus step-size at $100 \mathrm{~g} / \mathrm{cm}^{2}$ in water exposed to the August $1972 \mathrm{King}$ SPE. Both transport algorithms were used with six different energy grids.

While one would like to use the fully converged results in all future applications, the fine discretization parameters result in a very inefficient algorithm that would be impractical in most applications. Thus, a smaller grid and larger step-size is chosen, and the results generated with these coarse discretization parameters must be compared to the converged results to quantify the discretization error. As stated previously, HZETRN is commonly run with 100 energy grid points and a step-size of $0.5 \mathrm{~g} / \mathrm{cm}^{2}$. Therefore, $h=0.5 \mathrm{~g} / \mathrm{cm}^{2}$ and $N=100$ are chosen as the coarse discretization parameters. Define the converged solution as the results obtained with the new method using 753 energy grid points and a stepsize of $2^{-11} \mathrm{~g} / \mathrm{cm}^{2}$. This approximation is justified in Figures 13-16 where it appears that both methods are asymptotically approaching a common solution. It is also important to note that the difference between the methods with the fine discretization parameters is very small. The discretization error is expressed as the percent difference between the results obtained with the coarse parameters and the converged solution. The results are given in Figures 17 and 18. The new algorithm has a lower discretization error than the old algorithm out to $100 \mathrm{~g} / \mathrm{cm}^{2}$. Figures 17 and 18 also show that characteristically different errors are generated in aluminum and water targets. The data represented in Figures 17 and 18 are also given in Table 3 so that the exact numbers are clearly documented. Table 4 gives the error estimates for dose values.

Notice that in Figures 17 and 18, there is a slight bend in the error curves near $40 \mathrm{~g} / \mathrm{cm}^{2}$. This bend is the approximate depth at which the contribution to dose equivalent by the primary protons is overtaken by the contribution from secondary neutrons and charged target fragments, as shown in Figures 19 and 20. In aluminum, the error curve continues to increase past the bend, while in water, the error curve decreases past the bend. This is due to the number and energy of secondary neutrons and charged target fragments produced in each material. In aluminum, there are a moderate number of neutrons produced as a result of nuclear collisions between the primary protons and target media, and the many elastic collisions which dominate neutron transport result in only small energy transfers (compared to neutron collisions with hydrogen atoms in water). 


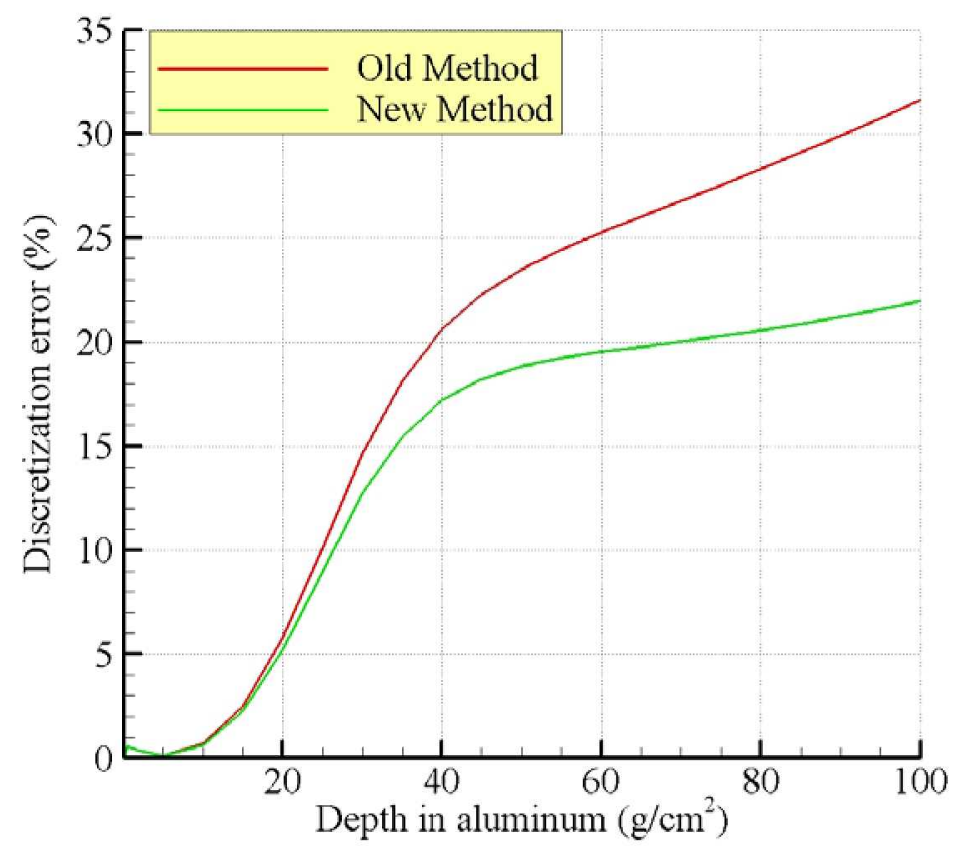

Figure 17. Discretization error for dose equivalent as a function of depth for the light particle transport algorithms in aluminum exposed to the August 1972 King SPE.

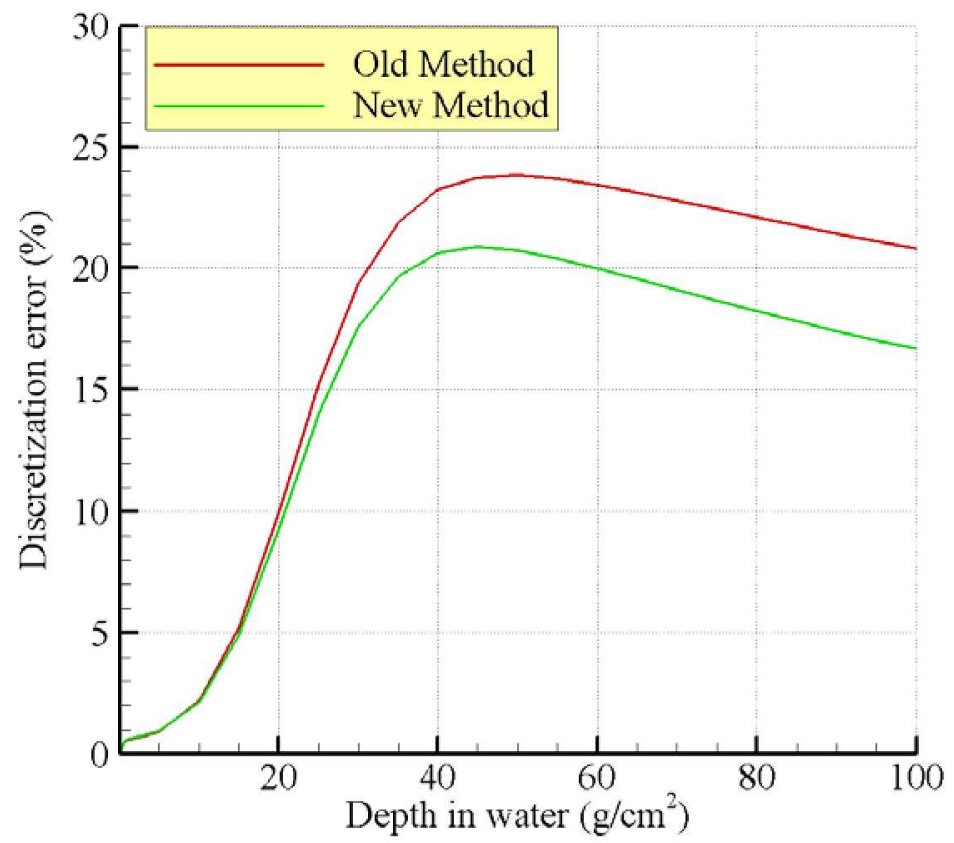

Figure 18. Discretization error for dose equivalent as a function of depth for the light particle transport algorithms in water exposed to the August 1972 King SPE. 
Table 3. Discretization error (\%) for dose equivalent computed with the old and new light particle transport algorithms at various depths in aluminum and water exposed to the August 1972 King SPE. Significant figures shown to clarify magnitudes.

\begin{tabular}{crrrr}
\hline \multirow{2}{*}{ Depth $\left(\mathrm{g} / \mathrm{cm}^{2}\right)$} & Old & New & Old & Wew \\
\hline 1.0 & 0.48 & 0.49 & 0.57 & 0.61 \\
5.0 & 0.08 & 0.10 & 0.95 & 0.97 \\
10.0 & 0.71 & 0.63 & 2.23 & 2.14 \\
25.0 & 10.10 & 8.94 & 15.24 & 14.01 \\
50.0 & 23.40 & 18.77 & 23.83 & 20.73 \\
75.0 & 27.42 & 20.18 & 22.43 & 18.65 \\
100.0 & 31.48 & 21.82 & 20.80 & 16.67 \\
\hline
\end{tabular}

Table 4. Discretization error (\%) for dose computed with the old and new light particle transport algorithms at various depths in aluminum and water exposed to the August 1972 King SPE. Significant figures shown to clarify magnitudes.

\begin{tabular}{crrrr}
\hline \multirow{2}{*}{ Depth $\left(\mathrm{g} / \mathrm{cm}^{2}\right)$} & Old & New & Old & New \\
\hline 1.0 & 0.09 & 0.79 & 0.31 & 0.37 \\
5.0 & 0.03 & 0.04 & 0.38 & 0.44 \\
10.0 & 0.11 & 0.11 & 0.49 & 0.51 \\
25.0 & 1.63 & 1.45 & 2.93 & 2.71 \\
50.0 & 17.49 & 14.56 & 15.43 & 13.65 \\
75.0 & 26.53 & 20.33 & 16.20 & 13.53 \\
100.0 & 30.54 & 22.04 & 14.51 & 11.46 \\
\hline
\end{tabular}

Thus, the secondary neutrons are left with sufficient energy to continue producing charged target fragments well past $40 \mathrm{~g} / \mathrm{cm}^{2}$. Conversely, in water, there are far fewer neutrons produced, and the elastic collisions result in much larger energy transfers that render many of the neutrons incapable of producing further target fragments. It is important to note that the error curves in Figure 17 do not grow without bound; they reach a maximum of about $75 \%$ near $725 \mathrm{~g} / \mathrm{cm}^{2}$ and then decline rapidly as the remaining neutrons have insufficient energy to produce any further target fragments. Error estimates for material thicknesses greater than $100 \mathrm{~g} / \mathrm{cm}^{2}$ are computationally expensive due to the long run-times associated with fine discretization parameters and large depths. This topic will be investigated in future work.

As stated previously, it is clear in Figures 15 and 16 that both methods, for a given energy grid, systematically under-predict dose equivalent values if step-sizes larger than $0.01 \mathrm{~g} / \mathrm{cm}^{2}$ are used. It is now shown that these step-sizes, including the coarse discretization parameter, $h=0.5 \mathrm{~g} / \mathrm{cm}^{2}$, commonly used in HZETRN, result in a systematic under-prediction of secondary target fragments, and that this underprediction is the dominant source of error shown in Figures 17 and 18. 


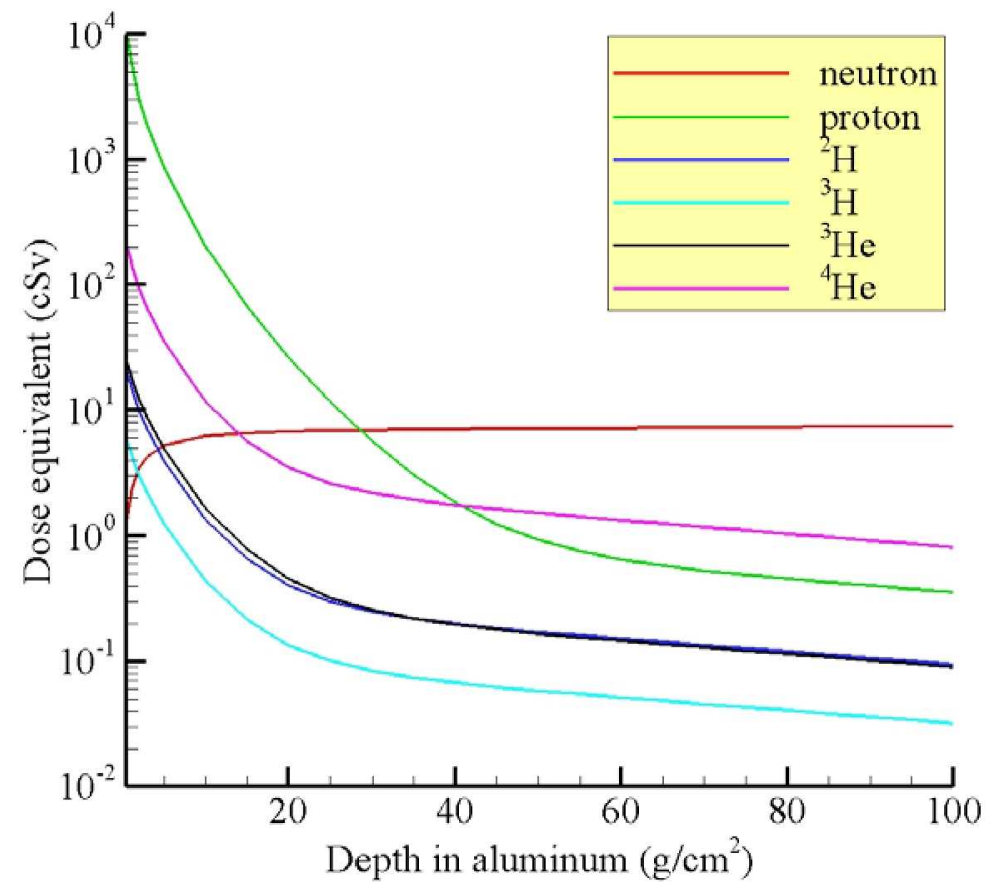

Figure 19. Dose equivalent by particle type using the converged results at $100 \mathrm{~g} / \mathrm{cm}^{2}$ in aluminum exposed to the August 1972 King SPE.

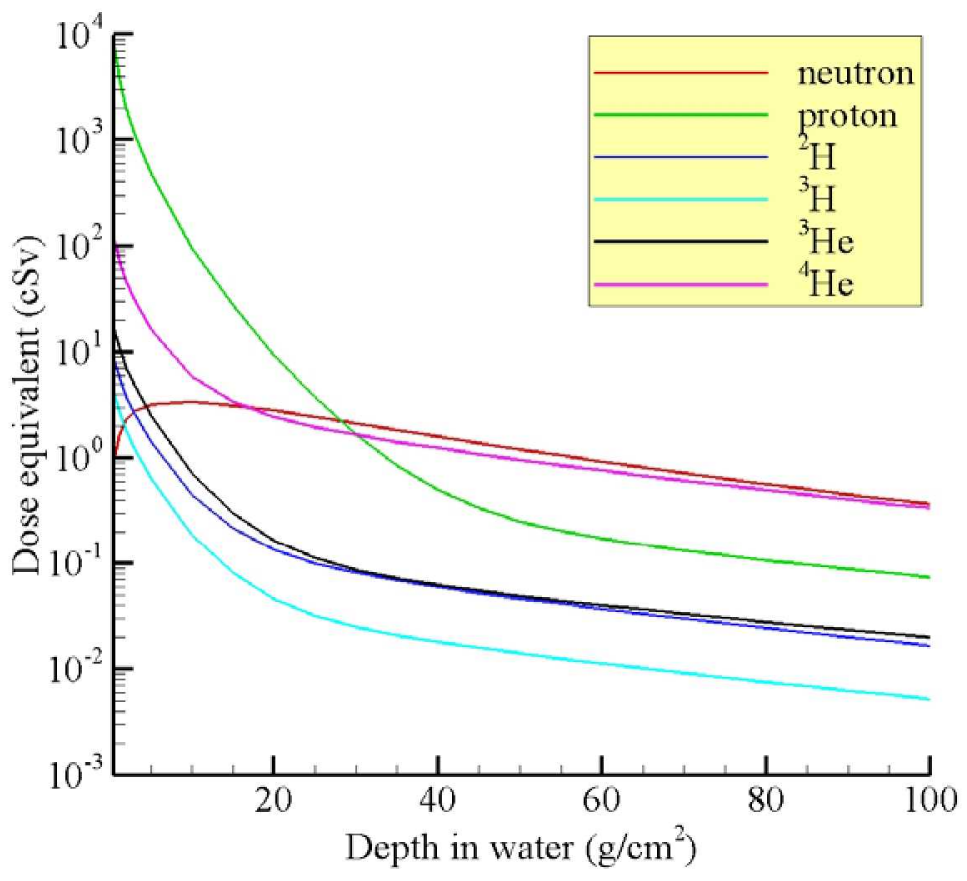

Figure 20. Dose equivalent by particle type using the converged results at $100 \mathrm{~g} / \mathrm{cm}^{2}$ in water exposed to the August 1972 King SPE. 
Recall from Section 5 that two convergence criteria were identified in developing numerical procedures for the light particle transport equation, namely that

$$
h \ll \frac{1}{\sigma_{j}(r)},
$$

and

$$
h \ll r_{j}
$$

The first criterion (step-size much smaller than the nuclear mean free path) is trivially satisfied since nuclear mean free path lengths are typically on the order of many $\mathrm{cm}$, but the second criterion (step-size much smaller than the range), which has been derived in the present work, is not satisfied for low energy particles unless $h$ is taken several orders of magnitude below $0.5 \mathrm{~g} / \mathrm{cm}^{2}$.

To show the error induced by choosing discretization parameters that violate equation (111), a portion of the convergence test outlined previously is re-run with the minimum energy increased to 50 AMeV. This energy was chosen because $50 \mathrm{MeV}$ protons have a range $\sim 2.9 \mathrm{~g} / \mathrm{cm}^{2}$, which should allow step-sizes of $0.5 \mathrm{~g} / \mathrm{cm}^{2}$ to be used with only minimal error. Since the systematic under-prediction has been shown to be independent of the numerical algorithm, only the new light particle transport equation was used. Also, since the error was most prevalent in aluminum, the water target was not considered. For this comparison, define the modified converged solution as the results generated by the new method with a step-size of $2^{-11} \mathrm{~g} / \mathrm{cm}^{2}$ and the 753 point modified energy grid $\left(E_{\min }=50 \mathrm{AMeV}\right.$ instead of $\left.0.01 \mathrm{AMeV}\right)$. The results generated by the new method using a step-size of $0.5 \mathrm{~g} / \mathrm{cm}^{2}$ and the modified 100 point energy grid will be compared to the modified converged results. The error comparisons are given in Figure 21 along with the previous error curve shown in Figure 17. It is clear that neglecting the target fragments with energy less than $50 \mathrm{AMeV}$ significantly reduces the discretization error in the light particle transport algorithm. The new error curve has a maximum of $\sim 0.07 \%$ near $30 \mathrm{~g} / \mathrm{cm}^{2}$ of aluminum.

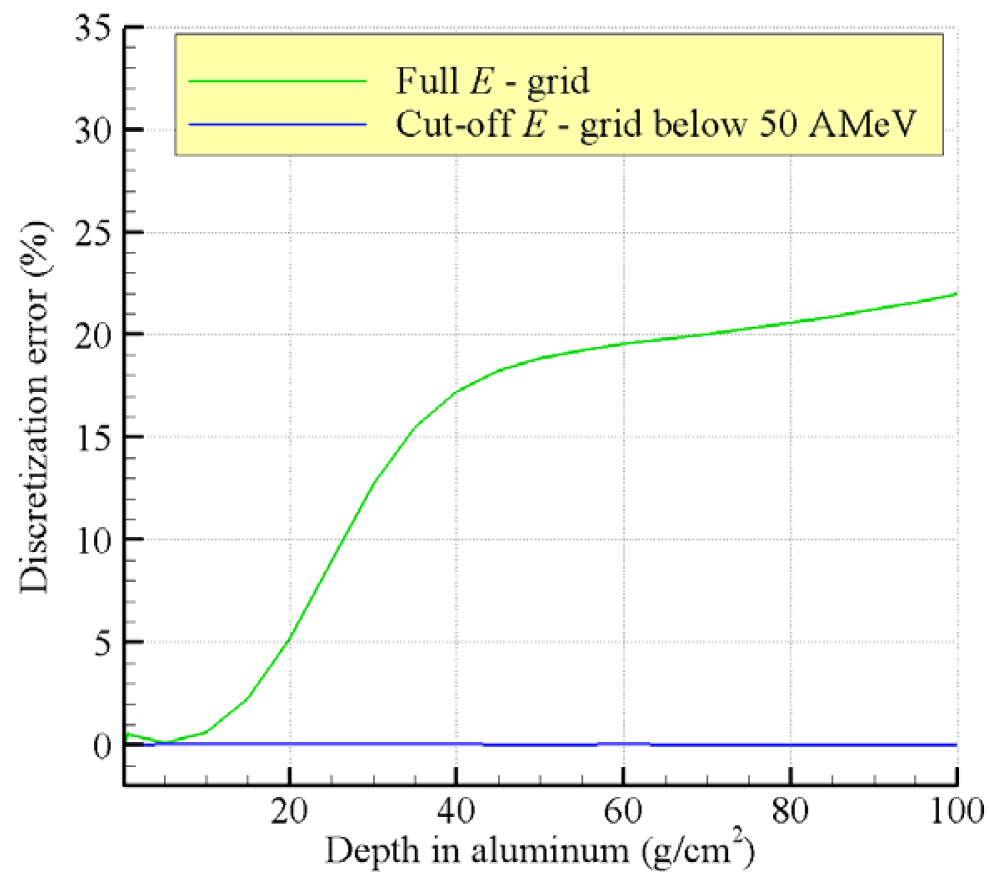

Figure 21. Discretization error (\%) caused by target fragments with energy less than $50 \mathrm{AMeV}$ in aluminum exposed to the August 1972 King SPE. 


\section{Convergence Study for HZETRN in GCR Environments}

For GCR environments, the primary spectrum is composed of protons, ${ }^{4} \mathrm{He}$, and 26 heavy ions with energies extending up to $50 \mathrm{AGeV}$. In HZETRN, the heavy ion transport algorithm is used to transport the 26 heavy ions along with their secondary fragments through the target media, and the light particle transport algorithm is used to transport the primary protons and ${ }^{4} \mathrm{He}$ along with the light projectile and target fragments. The light projectile fragments produced from the heavy ions are also included in the light particle transport algorithm. A convergence analysis has never been done, to our knowledge, for the heavy ion or light particle transport algorithms in GCR environments. The convergence analysis described in the previous section is repeated for the heavy ion and light particle transport algorithms for GCR boundary conditions.

In this section, the total discretization error associated with the heavy ion transport algorithm and the old and new light particle transport algorithms in HZETRN is examined by conducting a detailed convergence analysis in both step-size, $h$, and energy grid-size, $N$. For all comparisons, the round-off errors, coding mistakes, and interpolation routines mentioned in the previous section have been fixed in the original algorithms. This will allow a direct comparison between the original and updated numerical algorithms. The convergence analysis is identical to the one in Section 10 except that 1977 solar minimum GCR environment is used, and the maximum energy is increased to $E_{\max }=50 \mathrm{AGeV}$. The same step-sizes and energy grid-sizes are used. It is first shown that the heavy ion transport algorithm converges as a function of step-size and energy grid-size. The discretization error associated with the heavy ion transport algorithm for the discretization parameters $\left(h=0.5 \mathrm{~g} / \mathrm{cm}^{2}\right.$ with 100 energy grid points) commonly used in HZETRN is also given. Next, the two light particle transport algorithms are compared. The new algorithm is shown to converge as a function of step-size and energy grid-size. It is also determined that the old algorithm converges as a function of step-size, but that larger energy grids are required to determine if the old algorithm converges as a function of energy grid-size. Finally, total discretization error estimates are given for discretization parameters commonly used in HZETRN. The errors are expressed as percent difference from a converged numerical solution.

In Figures 22 and 23, dose equivalent as a function of depth in aluminum and water is given for the heavy ion transport algorithm coupled to both of the light particle transport algorithms and all of the discretization parameters. The plot legends have been removed because there are 132 different curves, many of which are overlapping. The spread in the data at $100 \mathrm{~g} / \mathrm{cm}^{2}$ in aluminum is $\sim 0.024 \mathrm{cSv} / \mathrm{day}$ $(\sim 17 \%)$, and the spread in the data at $100 \mathrm{~g} / \mathrm{cm}^{2}$ in water is $\sim 0.013 \mathrm{cSv} /$ day $(\sim 15 \%)$. As before, both methods with the various discretization parameters produce similar results, and the errors appear to be slowly increasing as a function of depth in the material. Slightly larger errors are once again found in aluminum. In Figures 24 and 25, the contribution to dose equivalent from heavy ions at $100 \mathrm{~g} / \mathrm{cm}^{2}$ in aluminum and water is plotted as a function of the energy grid-size, $N$, for five step-sizes. The step-sizes were chosen to reduce data overlap and improve plot clarity. In Figures 26 and 27, the contribution to dose equivalent from heavy ions at $100 \mathrm{~g} / \mathrm{cm}^{2}$ in aluminum and water is plotted as a function of the step-size, $h$, for all of the energy grids. Figures 24-27 clearly show that the heavy ion marching algorithm reaches an asymptotic solution for step-sizes less than $0.01 \mathrm{~g} / \mathrm{cm}^{2}$ and energy grids with greater than 250 points. It is concluded that the heavy ion transport algorithm converges as a function of step-size and energy grid-size.

In order to quantify the discretization error associated with the heavy ion transport algorithm, consider the contribution to dose equivalent from heavy ions only and define the converged heavy ion solution as the results obtained using 753 energy grid points and a step-size of $2^{-11} \mathrm{~g} / \mathrm{cm}^{2}$. The step-size, $h=$ $0.5 \mathrm{~g} / \mathrm{cm}^{2}$, and energy grid-size, $N=100$, are once again chosen as the coarse discretization parameters. The discretization error is expressed as the percent difference between the results generated with the coarse parameters and the converged solution. To be clear, these discretization error estimates were obtained using only the heavy ion contribution to dose equivalent. Thus, the error caused by the light particle transport algorithm is not included here. The results are shown in Figure 28 and 29 for the aluminum and water targets. The discretization errors are bounded over the depths shown by $0.3 \%$ in aluminum and $1.1 \%$ in water. Future work will focus on quantifying the discretization error depths greater than $100 \mathrm{~g} / \mathrm{cm}^{2}$. The data represented in Figures 17 and 18 are also given in Table 5 so that the exact numbers are clearly documented. Table 6 gives the error estimates for dose values. 


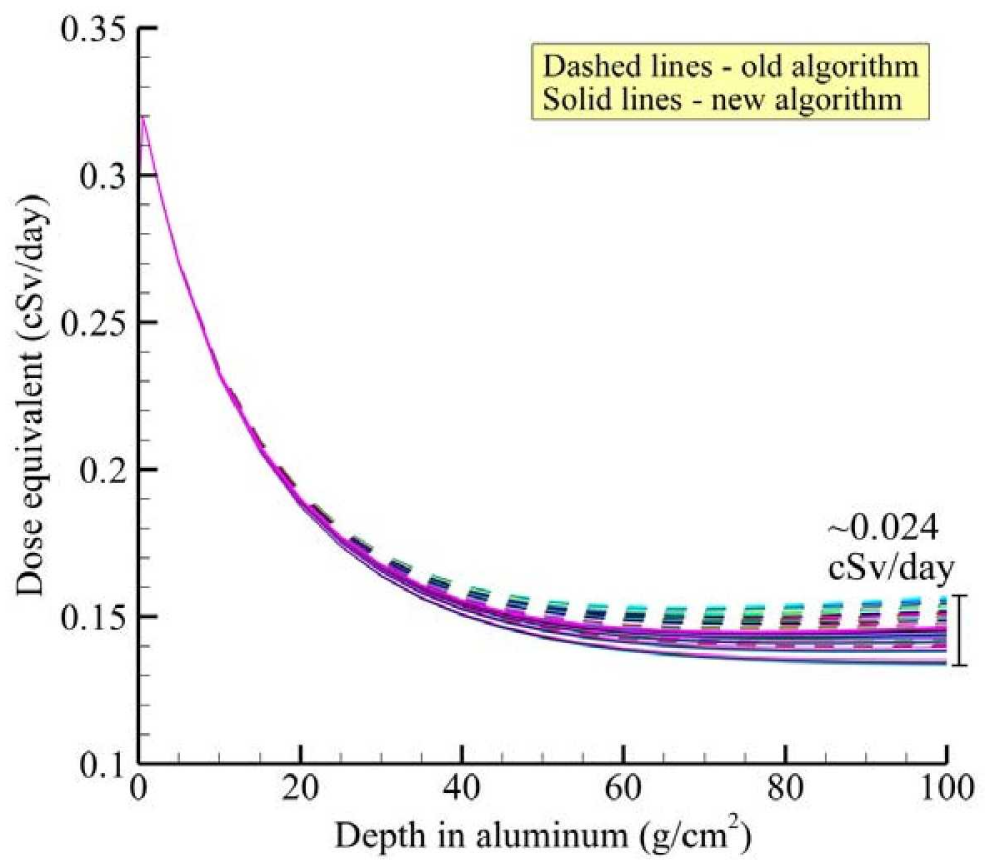

Figure 22. Dose equivalent versus depth in aluminum exposed to the 1977 solar minimum GCR computed with both transport algorithms and all discretization parameters.

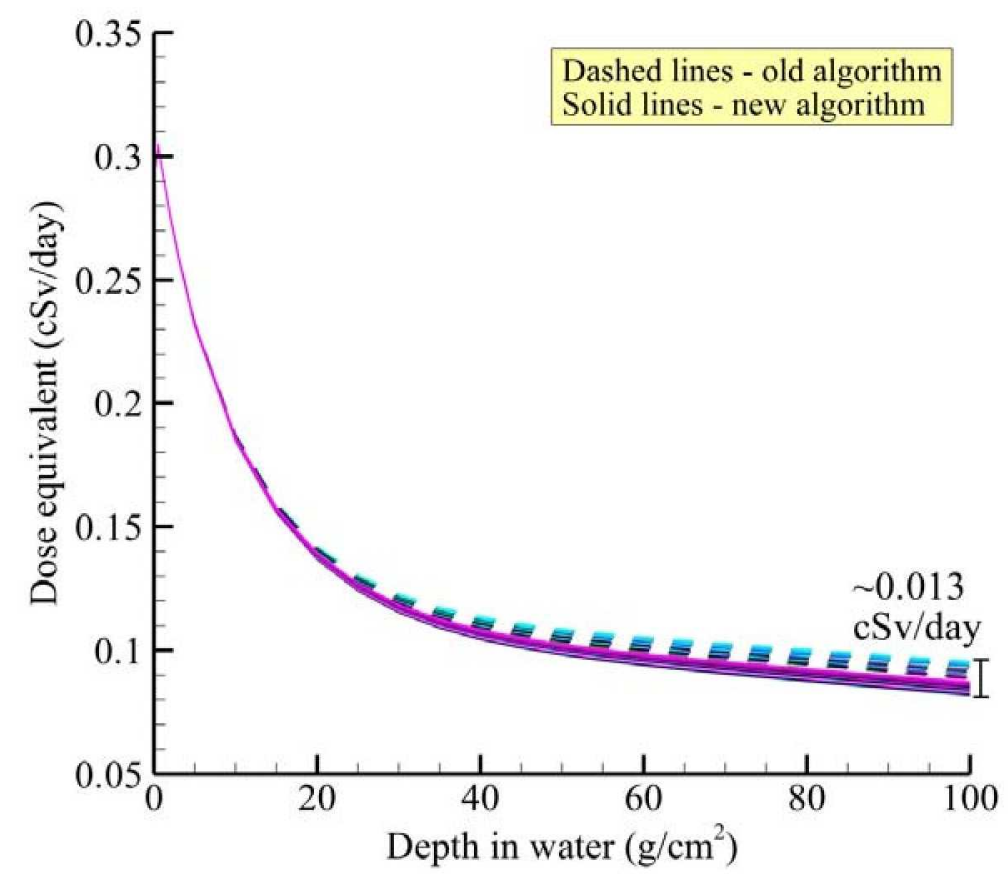

Figure 23. Dose equivalent versus depth in water exposed to the 1977 solar minimum GCR computed with both transport algorithms and all discretization parameters. 


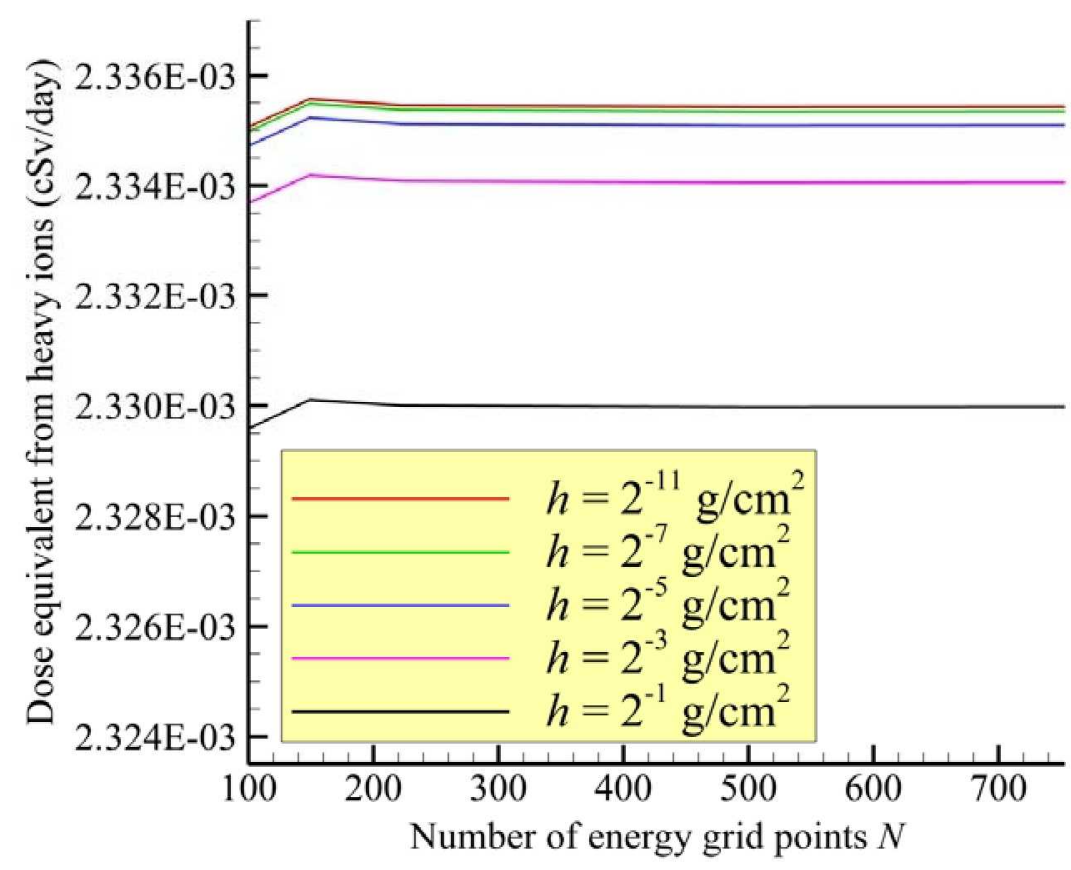

Figure 24. Dose equivalent from heavy ions versus energy grid-size at $100 \mathrm{~g} / \mathrm{cm}^{2}$ in aluminum exposed to the 1977 solar minimum GCR environment. Five different steps-sizes were used.

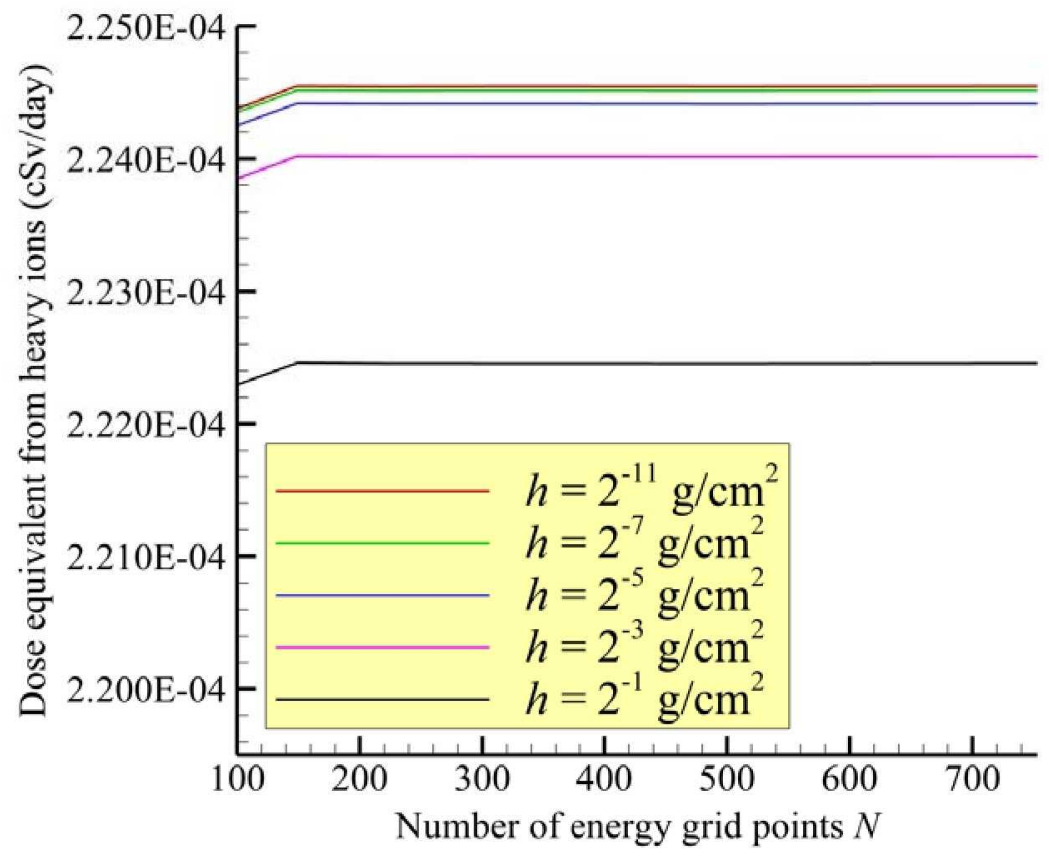

Figure 25. Dose equivalent from heavy ions versus energy grid-size at $100 \mathrm{~g} / \mathrm{cm}^{2}$ in water exposed to the 1977 solar minimum GCR environment. Five different steps-sizes were used. 


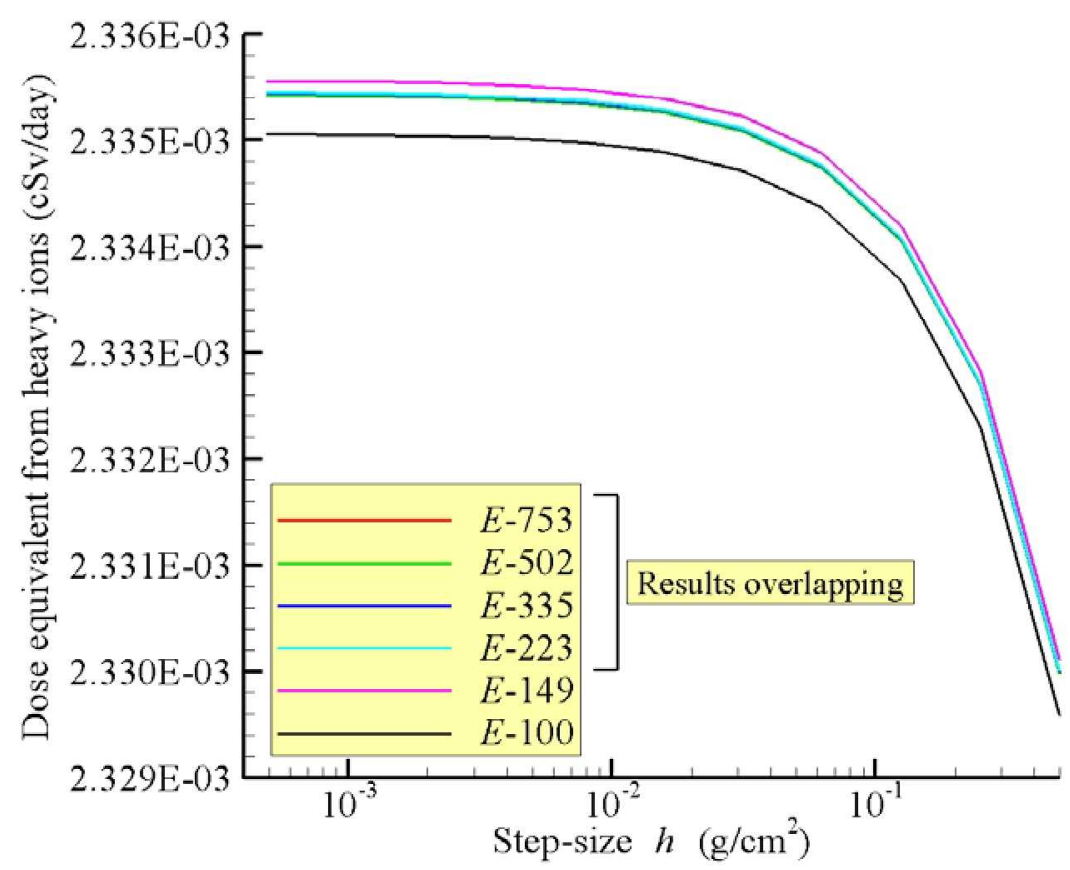

Figure 26. Dose equivalent from heavy ions versus step-size at $100 \mathrm{~g} / \mathrm{cm}^{2}$ in aluminum exposed to the 1977 solar minimum GCR environment. All of the different energy grids were used.

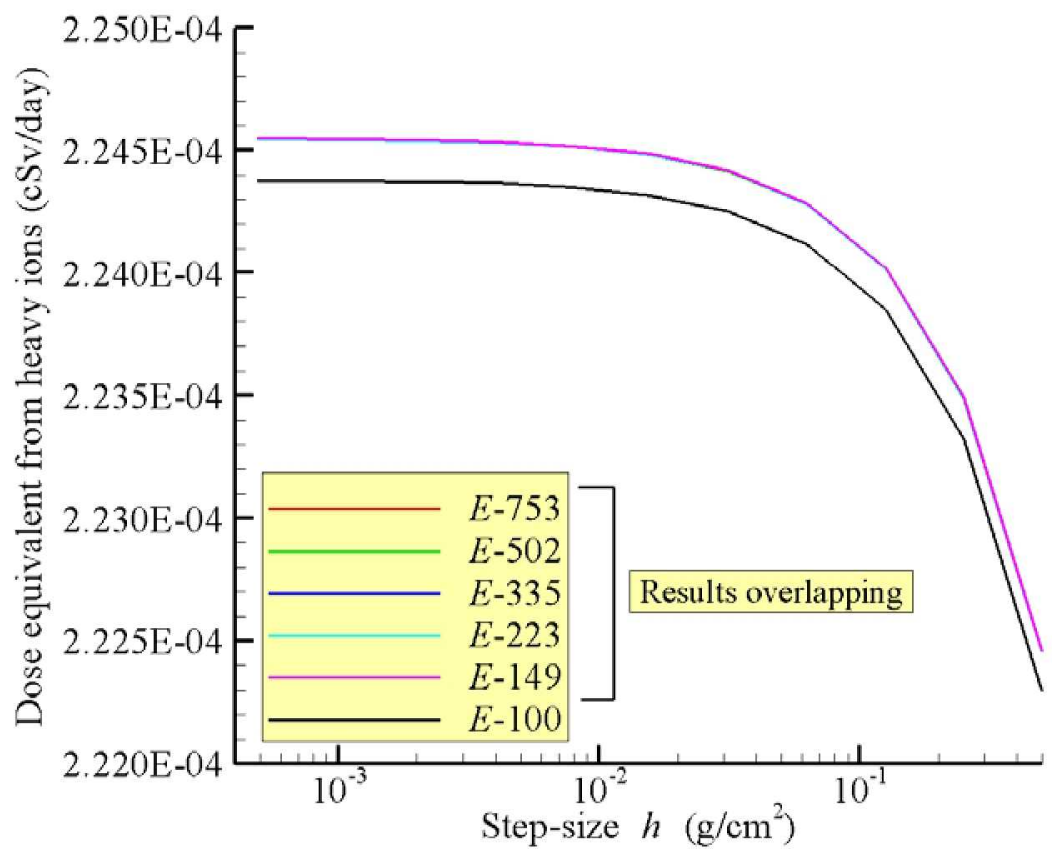

Figure 27. Dose equivalent from heavy ions versus step-size at $100 \mathrm{~g} / \mathrm{cm}^{2}$ in water exposed to the 1977 solar minimum GCR environment. All of the different energy grids were used. 


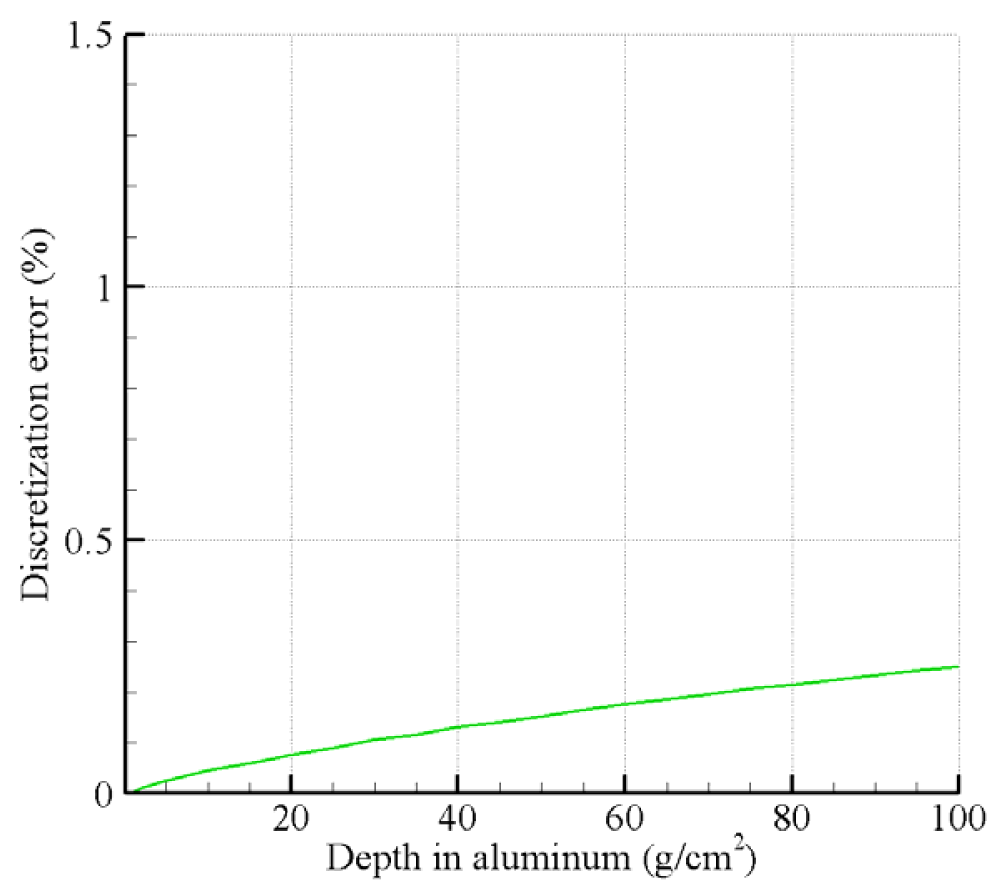

Figure 28. Discretization error for dose equivalent as a function of depth for the heavy ion transport algorithm in aluminum exposed to the 1977 solar minimum GCR environment. White space included so vertical axis matches Figure 29.

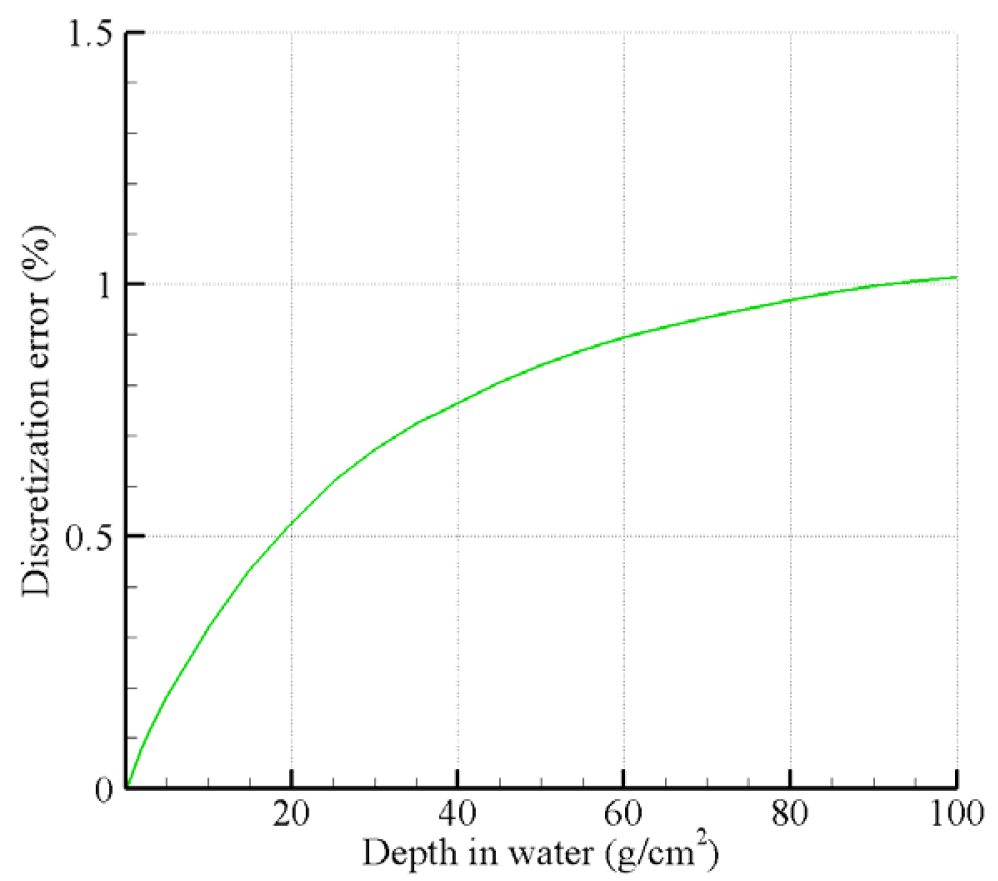

Figure 29. Discretization error for dose equivalent as a function of depth for the heavy ion transport algorithm in aluminum exposed to the 1977 solar minimum GCR environment. 
Table 5. Discretization error (\%) for the heavy ion contribution to dose equivalent computed with the heavy ion transport algorithm at various depths in aluminum and water exposed to the 1977 solar minimum GCR. Significant figures shown to clarify magnitudes.

\begin{tabular}{ccc}
\hline Depth $\left(\mathrm{g} / \mathrm{cm}^{2}\right)$ & Aluminum & Water \\
\hline 1.0 & 0.0026 & 0.037 \\
5.0 & 0.024 & 0.18 \\
10.0 & 0.045 & 0.32 \\
25.0 & 0.090 & 0.61 \\
50.0 & 0.15 & 0.84 \\
75.0 & 0.21 & 0.95 \\
100.0 & 0.25 & 1.01 \\
\hline
\end{tabular}

Table 6. Discretization error (\%) for the heavy ion contribution to dose computed with the heavy ion transport algorithm at various depths in aluminum and water exposed to the 1977 solar minimum GCR. Significant figures shown to clarify magnitudes.

\begin{tabular}{ccc}
\hline Depth $\left(\mathrm{g} / \mathrm{cm}^{2}\right)$ & Aluminum & Water \\
\hline 1.0 & 0.000327 & 0.020 \\
5.0 & 0.017 & 0.13 \\
10.0 & 0.035 & 0.23 \\
25.0 & 0.070 & 0.43 \\
50.0 & 0.12 & 0.61 \\
75.0 & 0.16 & 0.73 \\
100.0 & 0.20 & 0.82 \\
\hline
\end{tabular}

In Figures 30 and 31 , dose equivalent at $100 \mathrm{~g} / \mathrm{cm}^{2}$ in aluminum and water is plotted as a function of the energy grid-size, $N$, for three step-sizes. The step-sizes were chosen to reduce data overlap and improve plot clarity. In Figures 32 and 33, dose equivalent at $100 \mathrm{~g} / \mathrm{cm}^{2}$ in aluminum and water is plotted as a function of the step-size, $h$, for all of the energy grids. Figures 30-33 clearly show that the new light particle transport algorithm coupled to the heavy ion transport algorithm reaches an asymptotic solution for step-sizes less than $0.01 \mathrm{~g} / \mathrm{cm}^{2}$ and energy grids with greater than 300 points. It is concluded that the new light particle transport algorithm converges as a function of step-size and energy grid-size. Conversely, while the old algorithm appears to converge as a function of step-size, it has not converged as a function of energy grid-size if 753 grid points are used. This does not mean that the old algorithm will not converge. All that can be said is that an asymptotic solution is not achieved by the old algorithm if as many as 753 energy grid points are used. This would indicate that the new method is more accurate and converges faster as a function of energy grid-size.

In order to quantify the discretization error associated with the algorithms, define the converged solution as the results obtained with the new light particle transport algorithm (coupled to the heavy ion algorithm) using 753 energy grid points and a step-size of $2^{-11} \mathrm{~g} / \mathrm{cm}^{2}$. The step-size, $h=0.5 \mathrm{~g} / \mathrm{cm}^{2}$, and energy grid-size, $N=100$, are once again chosen as the coarse discretization parameters. The discretization error is expressed as the percent difference between the results generated with the coarse parameters and the converged solution. These error estimates were obtained using total exposure quantities (heavy ions and light particles included), thus the discretization error from the heavy ion transport algorithm, though small, is included. The results are shown in Figure 34 and 35 for the aluminum and water targets. The discretization errors are bounded over the depths shown by $6.5 \%$ in aluminum and $3.9 \%$ in water. The errors shown for the old method are misleadingly small. First, recall that the old method has not reached an asymptotic solution if 753 energy grid points are used; thus, it converges more slowly than the new algorithm as a function of energy grid-size. Second, the errors reported here are dependent on the choice of the converged solution. In this case, the energy discretization error and the spatial discretization error in the old algorithm are competing errors and therefore produce misleading error estimates when compared to a converged solution. 


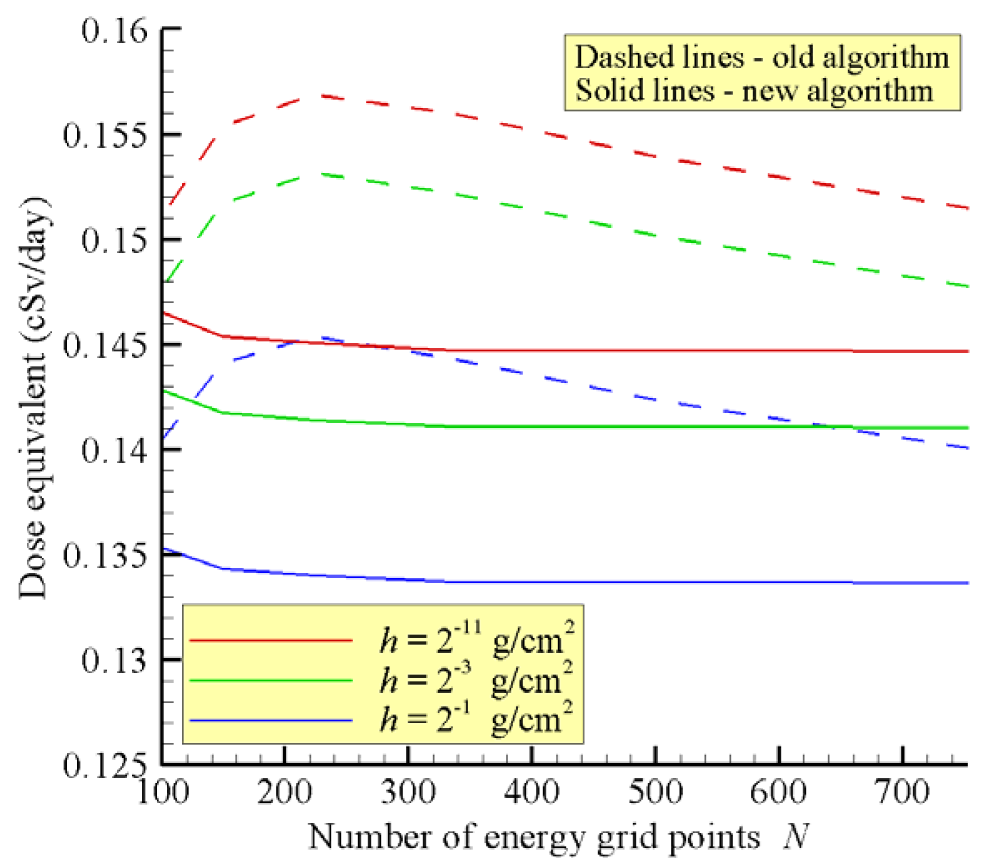

Figure 30. Dose equivalent versus energy grid-size at $100 \mathrm{~g} / \mathrm{cm}^{2}$ in aluminum exposed to the 1977 solar minimum GCR environment. Both transport algorithms were used with three different step-sizes.

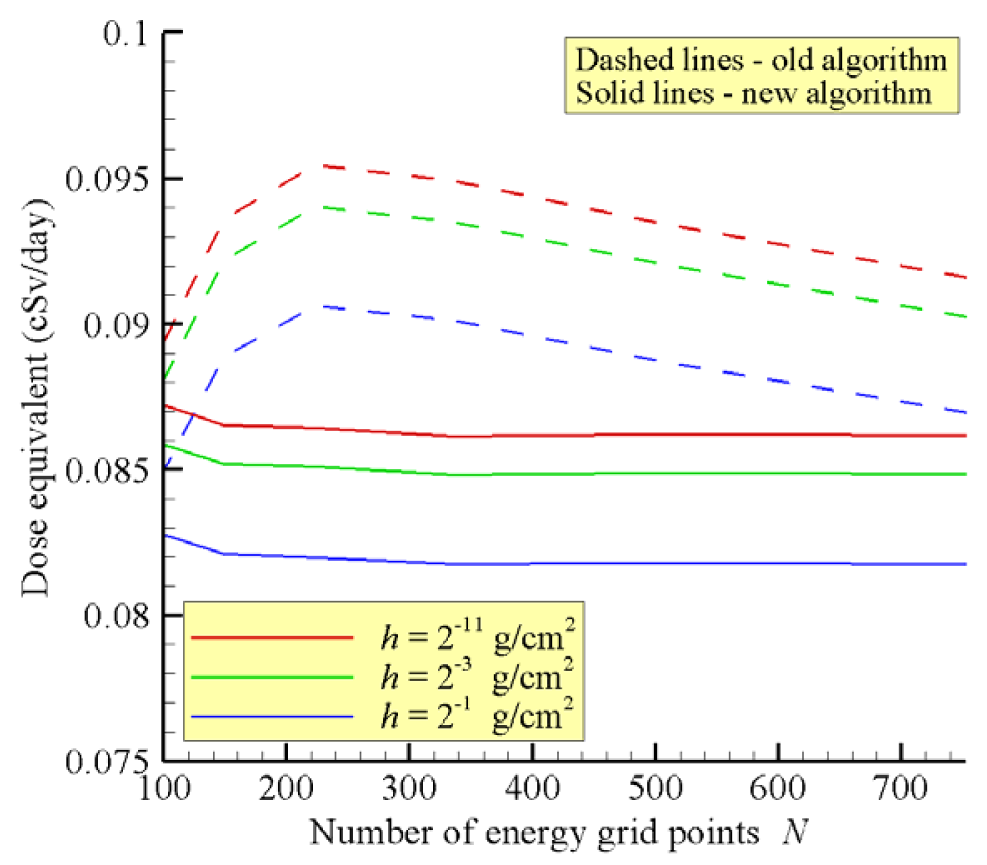

Figure 31. Dose equivalent versus energy grid-size at $100 \mathrm{~g} / \mathrm{cm}^{2}$ in aluminum exposed to the 1977 solar minimum GCR environment. Both transport algorithms were used with three different step-sizes. 


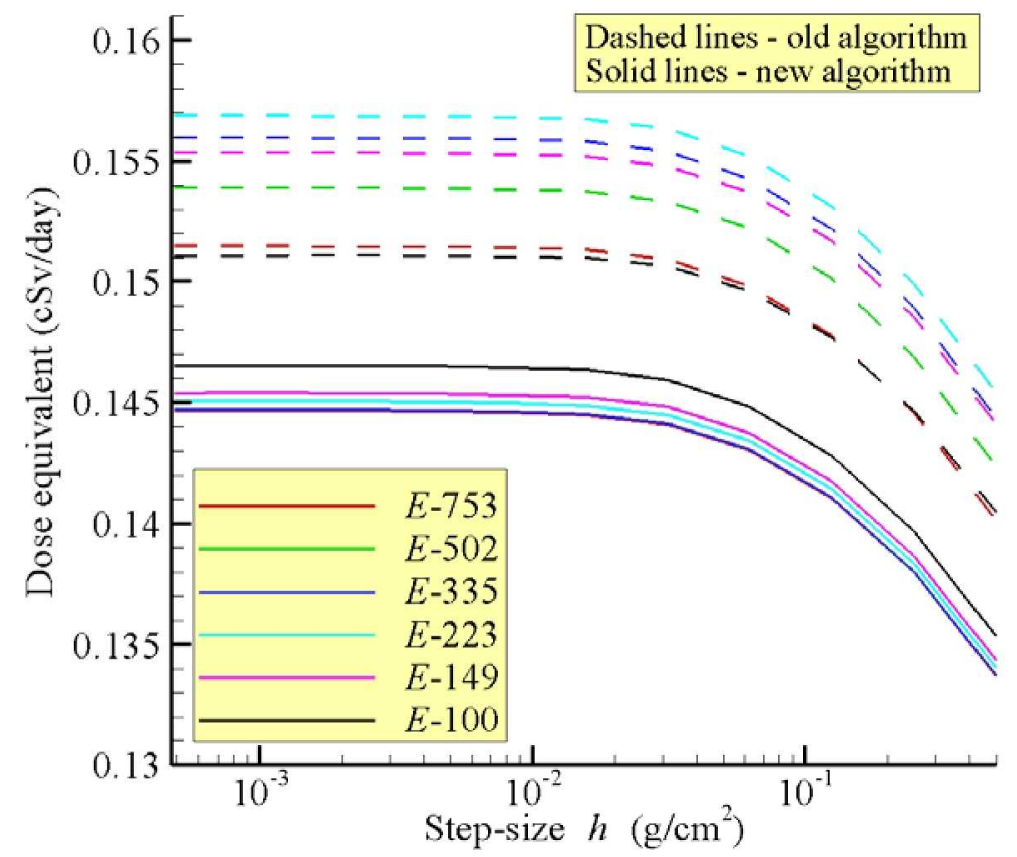

Figure 32. Dose equivalent versus step-size at $100 \mathrm{~g} / \mathrm{cm}^{2}$ in aluminum exposed to the 1977 solar minimum GCR environment. Both transport algorithms were used with six different energy grids.

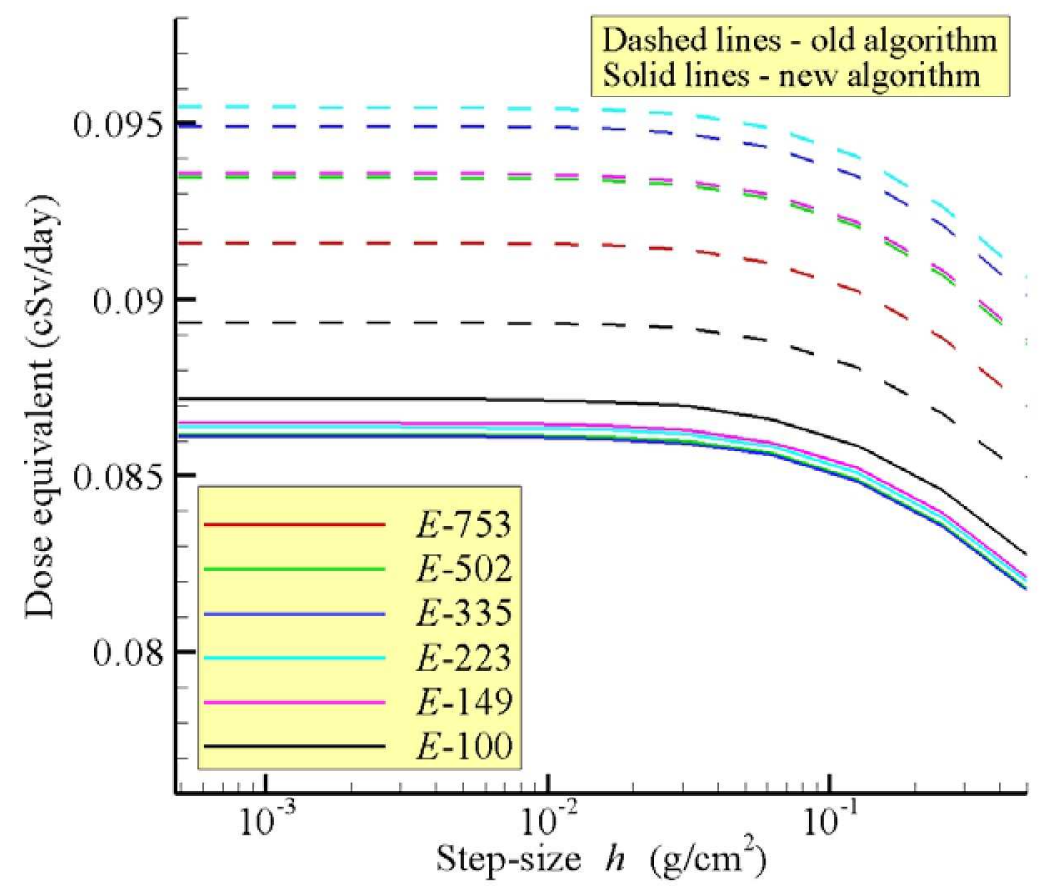

Figure 33. Dose equivalent versus step-size at $100 \mathrm{~g} / \mathrm{cm}^{2}$ in aluminum exposed to the 1977 solar minimum GCR environment. Both transport algorithms were used with six different energy grids. 


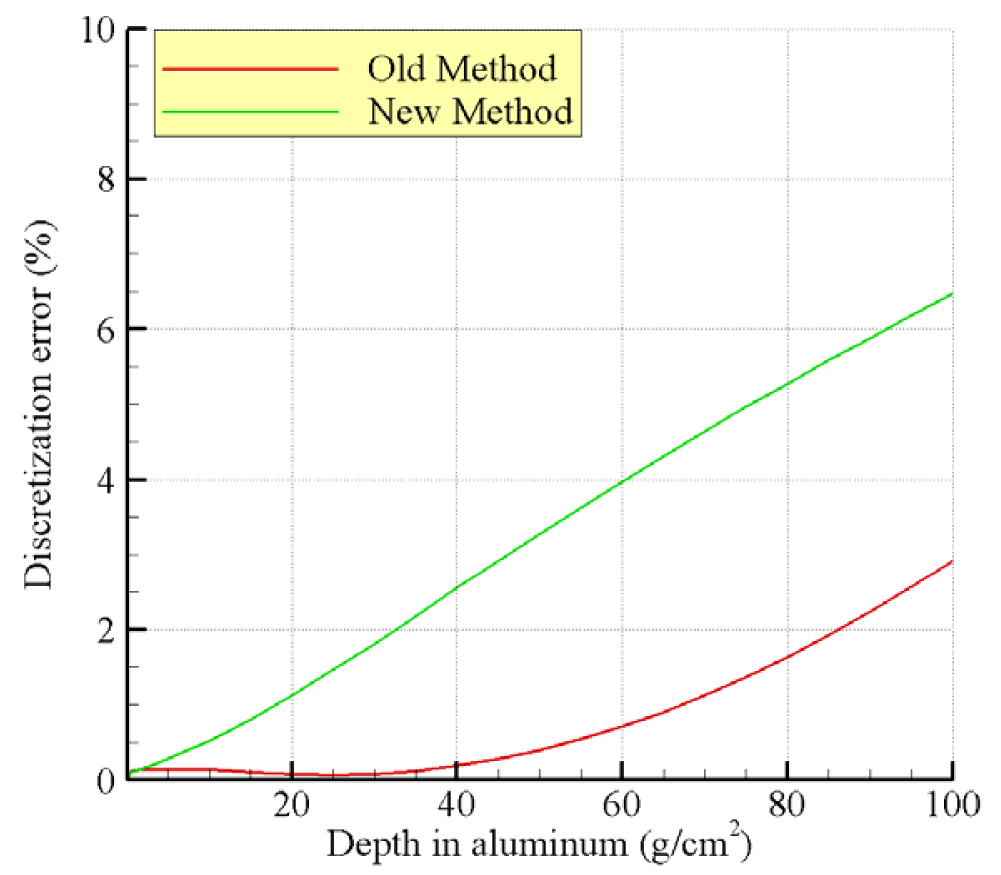

Figure 34. Discretization error (\%) for dose equivalent as a function of depth for both algorithms in aluminum exposed to the 1977 solar minimum GCR environment.

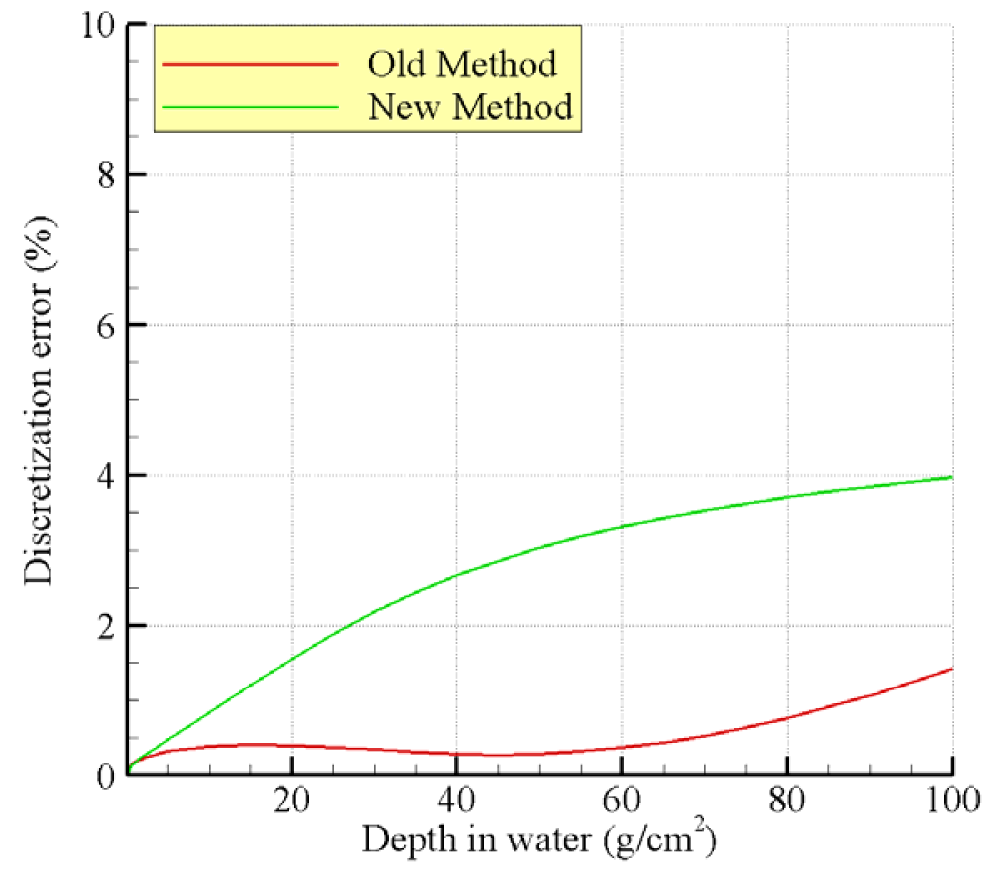

Figure 35. Discretization error (\%) for dose equivalent as a function of depth for both algorithms in water exposed to the 1977 solar minimum GCR environment. 
As before, the errors are slowly increasing as a function of depth in the material, and it is expected that these errors will reach a maximum after the majority of the primary ions and secondary projectile fragments have come to rest. Future work will focus on quantifying the discretization error depths greater than $100 \mathrm{~g} / \mathrm{cm}^{2}$. The data represented in Figures 34 and 35 is also given in Tables 7. Table 8 gives the error estimates for dose.

Table 7. Total discretization error (\%) for dose equivalent computed with both algorithms at various depths in aluminum and water exposed to the 1977 solar minimum GCR. Significant figures shown to clarify magnitudes.

\begin{tabular}{rcccc}
\hline $\begin{array}{c}\text { Depth } \\
\left(\mathrm{g} / \mathrm{cm}^{2}\right)\end{array}$ & Old & New & Old & Water \\
\hline 1.0 & 0.13 & 0.12 & 0.17 & 0.17 \\
5.0 & 0.15 & 0.28 & 0.31 & 0.47 \\
10.0 & 0.14 & 0.52 & 0.39 & 0.84 \\
25.0 & 0.07 & 1.46 & 0.37 & 1.88 \\
50.0 & 0.40 & 3.27 & 0.28 & 3.03 \\
75.0 & 1.37 & 4.96 & 0.63 & 3.62 \\
100.0 & 2.92 & 6.47 & 1.42 & 3.97 \\
\hline
\end{tabular}

Table 8. Total discretization error (\%) for dose computed with both algorithms at various depths in aluminum and water exposed to the 1977 solar minimum GCR. Significant figures shown to clarify magnitudes.

\begin{tabular}{rcccc}
\hline $\begin{array}{c}\text { Depth } \\
\left(\mathrm{g} / \mathrm{cm}^{2}\right)\end{array}$ & Old & New & Old & Wew \\
\hline 1.0 & 0.04 & 0.07 & 0.07 & 0.11 \\
5.0 & 0.16 & 0.12 & 0.10 & 0.24 \\
10.0 & 0.48 & 0.18 & 0.41 & 0.33 \\
25.0 & 1.53 & 0.32 & 1.36 & 0.46 \\
50.0 & 3.09 & 0.56 & 2.49 & 0.55 \\
75.0 & 4.25 & 0.83 & 2.80 & 0.56 \\
100.0 & 4.91 & 1.11 & 2.39 & 0.53 \\
\hline
\end{tabular}

\section{Run Time Comparisons}

In this section, the run-times of the old and new transport algorithms are compared for three applications in which HZETRN is commonly used. All simulations were run on a Dell with 4 quad-core AMD Opteron Processor 8356 chips and 131 GB of memory. The discretization parameters used in each case where $h=0.5 \mathrm{~g} / \mathrm{cm}^{2}$ and 100 energy grid points. The simplest of the three applications would be to use HZETRN to compute flux/fluence, dose, or dose equivalent as a function of depth in a single layer of some material. This type of calculation might be done to analyze the radiation protection properties of a slab of some material or to generate a simplified interpolation database. In this case, consider separately aluminum exposed to the August 1972 King SPE and the 1977 solar minimum GCR spectrum. For the SPE environment, results are stored at 21 depths from $0.0 \mathrm{~g} / \mathrm{cm}^{2}$ to $100.0 \mathrm{~g} / \mathrm{cm}^{2}$. For the GCR environment, results are stored at 11 depths from $0.0 \mathrm{~g} / \mathrm{cm}^{2}$ to $100.0 \mathrm{~g} / \mathrm{cm}^{2}$. The difference in the number of depths has been discussed by Anderson et al. [2007] and is related to the rapid decline of the SPE dosimetric quantities over the first few $\mathrm{g} / \mathrm{cm}^{2}$ of shielding. The run-times are given in Table 9. The next application is a twolayer simulation in which results are stored on a two dimensional grid of points covering every combination of first layer thicknesses followed by second layer thicknesses. In this case, consider a first layer of aluminum followed by a second layer of water. This simulation is typically used to model a shielding 
structure (aluminum) surrounding a human phantom (water). The spatial grids mentioned above for the SPE and GCR environments are used in this application. The run-times are given in Table 10. The final application is a three-layer simulation in which results are stored on a three dimensional grid of points covering every combination of first, second, and third layer thicknesses. In this case, consider a first layer of aluminum, a second layer of polyethylene, and a third layer of water. This simulation is typically used to model a shielding structure (aluminum) with a secondary shield (polyethylene) surrounding a human phantom (water). The spatial grids mentioned above for the SPE and GCR environments are used in this application. The run-times are given in Table 11.

Table 9. Single layer run time comparisons for old and new algorithms in SPE and GCR environments. The reduction factor is the ratio of the old method run-time to the new method run-time.

\begin{tabular}{lcc}
\hline & SPE time $(\mathrm{sec})$ & GCR time $(\mathrm{sec})$ \\
\hline Old Method & 62.0 & 77.0 \\
New Method & 0.7 & 9.0 \\
\hline Reduction Factor & 88.5 & 8.5 \\
\hline
\end{tabular}

Table 10. Two layer run time comparisons for old and new algorithms in SPE and GCR environments. The reduction factor is the ratio of the old method run-time to the new method run-time.

\begin{tabular}{lcc}
\hline & SPE time $(\mathrm{sec})$ & GCR time $(\mathrm{sec})$ \\
\hline Old Method & 1272 & 926 \\
New Method & 15 & 108 \\
\hline Reduction Factor & 85 & 9 \\
\hline
\end{tabular}

Table 11. Three layer run time comparisons for old and new algorithms in SPE and GCR environments. The reduction factor is the ratio of the old method run-time to the new method run-time.

\begin{tabular}{lcc}
\hline & SPE time $(\mathrm{min})$ & GCR time $(\mathrm{min})$ \\
\hline Old Method & 440 & 173 \\
New Method & 5 & 21 \\
\hline Reduction Factor & 88 & 8 \\
\hline
\end{tabular}

The run-times in Tables 9-11 show that the new method is much faster than the old method in both SPE and GCR environments. The new method is nearly 90 times faster for SPE simulations and nearly 10 times faster for GCR simulations. It should be noted that run-times in the old method are dependent on the atomic complexity of the target material. For targets with many atomic species, the cross sections computed within the transport procedure will require more time and therefore slow the transport procedure. However, in the new method, cross sections are pre-computed, and therefore, increased atomic complexity does not affect the run times. Since the materials considered here had at most two atomic species (water, polyethylene), the run times for the old method and reduction factors are both conservative.

\section{Conclusions and Future Work}

In this paper, a detailed derivation of the numerical marching algorithms used for light particles and heavy ions in HZETRN was given. Components of the derivation that were previously absent in the documentation were discussed in detail. This work serves as a complete set of documentation for the old and new numerical methods used in the straight ahead transport procedures in HZETRN. A new numerical method was presented for the light particle transport algorithm. The primary differences between the old 
and new algorithms were summarized in process description charts and tables. In general, the new method requires fewer interpolations per step.

The impact of single precision round-off error and various coding errors on code stability and accuracy was discussed. Round-off error was shown to be problematic for some of the light particle production cross sections. These errors produce discontinuities in the high energy portion of secondary light particle fluence spectra. Three coding errors related to the light particle production cross sections were discussed, and the individual and combined effect of the errors on fluence spectra was shown. A new interpolation routine used in both the heavy ion and light particle transport algorithm was presented, and the improved efficiency and robustness of the routine was demonstrated.

A convergence study was conducted to determine if the old and new algorithms converge as a function of step-size and energy grid-size and to quantify the discretization error associated with both algorithms. The old and new algorithms were shown to converge to the same result for the SPE environment, and the improved accuracy of the new method was quantified. Total discretization error on dose equivalent at $100 \mathrm{~g} / \mathrm{cm}^{2}$ was reduced from $31 \%$ to $21 \%$ in aluminum and from $20 \%$ to $15 \%$ in water for commonly used energy grids. For the GCR environment, the heavy ion transport algorithm was first shown to converge as a function of step-size and energy grid-size, and discretization error estimates were bounded over the depths shown by approximately $1 \%$ at $100 \mathrm{~g} / \mathrm{cm}^{2}$ in aluminum and water. The new light particle transport algorithm was also shown to converge as a function of step-size and energy grid-size. The old algorithm was shown to converge as a function of step-size, and it was concluded that energy grids with more than 753 points are required to show convergence in the energy domain. This indicates that the new method converges faster and is more accurate in GCR environments. Total discretization error on dose equivalent at $100 \mathrm{~g} / \mathrm{cm}^{2}$ was $6 \%$ in aluminum and $4 \%$ in water for the new method.

The importance of low energy target fragments on discretization error was clearly shown by neglecting all particles with energy less than $50 \mathrm{AMeV}$ in the transport procedure. The error estimates for the cut-off energy grid were negligible compared to the full energy grid. This indicates that the dominant source of discretization error in HZETRN is caused by low energy target fragmentation. Further, to the author's knowledge, only the convergence criterion in equation (110) (step-size smaller than the nuclear mean free path length) has been addressed in the literature. The second convergence criterion in equation (111) (step-size smaller than the fragment range), found here in the derivation of the numerical procedures, has never been explicitly written until now. This work definitively shows the presence of two convergence criteria, and that previous convergence studies never addressed the second criterion.

Run time comparisons between the old and new algorithms showed large improvements for three applications in which HZETRN is commonly used. The new algorithm was found to be approximately 90 times faster for SPE simulations and approximately 10 times faster for GCR simulations. The new algorithms will be implemented in all future versions of HZETRN.

Future work should focus on further reducing discretization error in HZETRN by developing more robust methods for handling the transport of low energy target fragments. While physics modeling errors associated with the straight ahead approximation may exceed the current numerical error at depths past 100 $\mathrm{g} / \mathrm{cm}^{2}$, there is still a need to reduce the numerical error as long as the current model is used in studies with such large material depths (atmosphere, lunar surface, martian surface, etc.).

\section{Acknowledgements}

This research was sponsored by NASA Research Grant NNL06AA14A and NNX09AR20A. The authors would like to thank William Oberkampf for his guidance and helpful discussions.

\section{References}

Anderson, B., Blattnig, S.R., Clowdsley, M.S., Numerical Uncertainty Quantification for Radiation Analysis Tools. Society of Automotive Enginneers International Conference on Environmental Systems, 07ICES-92 (2007). 
Bethe, Von H., Zur Theorie des Durchgangs schneller Korpuskularstraheln durch Materie., Ann. Phys., 5 Folge, Bd. 5, pp. 325-400 (1930).

Cucinotta, F.A., Calculations of Cosmic-Ray Helium Transport in Shielding Materials. NASA Technical Paper 3354 (1993).

Cucinotta, F.A., Townsend, L.W., Wilson, J.W., Shinn, J.L., Badhwar, G.D., Dubey, R.R., Light Ion Components of the Galactic Cosmic Rays: Nuclear Interactions and Transport Theory. Advances in Space Research, Volume 17, pp. 77-86 (1996).

Cucinotta, F.A., Wilson, J.W., Saganti, P., Hu, X., Kim, M.Y., Cleghorn, T., Zeitlin, C., Tripathi, R.K., Isotopic Dependence of GCR Fluence behind Shielding. Radiation Measurements, Volume 41, pp. 1235-1249 (2006).

Haberman, R., Elementary Applied Partial Differential Equations. Prentice Hall, New Jersey (1998).

Heinbockel, J.H., Slaba, T.C., Blattnig, S.R., Tripathi, R.K., Townsend, L.W., Handler, T., Gabriel, T.A., Pinsky, L.S., Reddell, B., Clowdsley, M.S., Singleterry, R.C., Norbury, J.W., Badavi, F.F., Aghara, S.K., Comparison of the Radiation Transport Codes HZETRN, HETC-HEDS and FLUKA using the February 1956 Webber SPE spectrum. NASA Technical Paper 2009-215560 (2009a).

Heinbockel, J.H., Slaba, T.C., Tripathi, R.K., Blattnig, S.R., Norbury, J.W., Badavi, F.F., Townsend, L.W., Handler, T., Gabriel, T.A., Pinsky, L.S., Reddell, B., Aumann, A.R., Comparison of the Transport Codes HZETRN, HETC and FLUKA using the 1977 GCR Solar Minimum Spectra. NASA Technical Paper 2009-215941 (2009b).

King, J.H., Solar Proton Fluences for 1977-1983 Space Missions. Journal of Spacecraft and Rockets, Volume 11,pp. 401-408 (1974).

Lamkin, S.L., Khandelwal, G.S., Shinn, J.L., Wilson, J.W., Numerical Methods for High Energy Nucleon Transport. Proceedings of the Topical Meeting on New Horizons in Radiation Protection and Shielding, Report Number OCLC 26290867 (1992).

Lamkin, S.L., Khandelwal, G.S., Shinn, J.L., Wilson, J.W., Space Proton Transport in One Dimension. Nuclear Science and Engineering, Volume 116, pp. 291-299 (1994).

Marchuk, G.I. and Lebedev, V.I., Numerical Methods in the Theory of Neutron Transport. Harwood Academic Publishers, New York (1986).

NASA, NASA Standards for Modeling and Simulation, NASA Standard 7009 (2008).

O'Neill, P.M., Badhwar-O'Neill, Galactic Cosmic Ray Model Update Based on Advanced Composition Explorer (ACE) Energy Spectra from 1997 to Present. Advances in Space Research, Volume 37, pp. 1727-1733 (2006).

Roy, C.J., Review of Code and Solution Verfication Procedures for Computational Simulation. Journal of Computational Physics, Volume 205, pp. 131-156 (2005).

Shinn, J.L., Wilson, J.W., Weyland, M., Cucinotta, F.A., Improvements in the Computational Accuracy of BRYNTRN (A Baryon Transport Code). NASA Technical Paper 3093 (1991).

Shinn, J.L., John, S., Tripathi, R.K., Wilson, J.W., Townsend, L.W., Norbury, J.W., A Fully EnergyDependent HZETRN (A Galactic Cosmic-Ray Transport Code). NASA Technical Paper 3243 (1992). 
Slaba, T.C., Qualls, G.D., Clowdsley, M.S., Blattnig, S.R., Simonsen, L.C., Walker, S.A., Singleterry, R.C., Analysis of Mass Averaged Tissue Doses in CAM, CAF, MAX, and FAX. NASA Technical Paper 2009-215562 (2009).

Slaba, T.C., Blattnig, S.R., Clowdsley, M.S., Walker, S.A., Badavi, F.F., An Improved Neutron Transport Algorithm for HZETRN. Advances in Space Research, Accepted (2010).

Wade, W.R., An Introduction to Analysis. Prentice Hall, Upper Saddle River, NJ (2000).

Wilson, J.W. and Lamkin, S.L., Perturbation Theory for Charged-Particle Transport in One Dimension. Nuclear Science and Engineering, Volume 57, pp. 292-299 (1975).

Wilson, J.W., Analysis of the Theory of Heavy Ion Transport. NASA Technical Note D-8381 (1977).

Wilson, J.W. and Badavi, F.F., Methods of Galactic Heavy Ion Transport. Radiation Research, Volume 108, pp. 231-237 (1986).

Wilson, J.W. and Townsend, L.W., A Benchmark for Galactic Cosmic-Ray Transport Codes. Radiation Research, Volume 114, pp. 201-206 (1988).

Wilson, J.W., Townsend, L.W., Nealy, J.E., Chun, S.Y., Hong, B.S., Buck, W.W., Lamkin, S.L., Ganapol, B.D., Khan, F., Cucinotta, F.A., BRYNTRN: A Baryon Transport Model. NASA Technical Paper 2887 (1989).

Wilson, J.W., Townsend, L.W., Schimmerling, W., Khandelwal, G.S., Khan, F., Nealy, J.E., Cucinotta, F.A., Simonsen, L.C., Shinn, J.L., Norbury, J.W., Transport Methods and Interactions for Space Radiations. NASA Reference Publication 1257 (1991).

Wilson, J.W., Badavi, F.F., Cucinotta, F.A., Shinn, J.L., Badhwar, G.D, Silberberg, R., Tsao, C.H., Townsend, L.W., Tripathi, R.K., HZETRN: Description of a Free-Space Ion and Nucleon Transport and Shielding Computer Program. NASA Technical Paper 3495 (1995).

Wilson, J.W., Tripathi, R.K., Mertens, C.J., Blattnig, S.R., Clowdsley, M.S., Cucinotta, F.A., Tweed, J., Heinbockel, J.H., Walker, S.A., Nealy, J.E., Verification and Validation: High Charge and Energy (HZE) Transport Codes and Future Development. NASA Technical Paper 2005-213784 (2005).

Wilson, J.W., Tripathi, R.K., Badavi, F.F., Cucinotta, F.A., Standardized Radiation Shield Design Method: 2005 HZETRN. SAE ICES 2006-18 (2006). 


\section{Appendix A - Inversion of Light Particle Transport Equation}

In this section, the scaled light particle transport equation (equation (23) in Section 3)

$$
\left[\frac{\partial}{\partial x}-\nu_{j} \frac{\partial}{\partial r}+\sigma_{j}(r)\right] \psi_{j}(x, r)=\sum_{k} \frac{\bar{\nu}_{j}}{\bar{\nu}_{k}} \int_{r}^{\infty} s_{j k}\left(r, r^{\prime}\right) \psi_{k}\left(x, r^{\prime}\right) d r^{\prime}
$$

is inverted to obtain a Volterra type integral equation.

For light ions only (neutrons will be handled separately later), define the characteristic variables

$$
\eta_{j} \equiv r-\nu_{j} x
$$

and

$$
\xi_{j} \equiv r+\nu_{j} x
$$

The differentials with respect to $x$ and $r$ on the left hand side of the light particle transport equation are transformed as

$$
\frac{\partial}{\partial x}=\frac{\partial}{\partial \eta_{j}} \frac{\partial \eta_{j}}{\partial x}+\frac{\partial}{\partial \xi_{j}} \frac{\partial \xi_{j}}{\partial x}=\nu_{j}\left(\frac{\partial}{\partial \xi_{j}}-\frac{\partial}{\partial \eta_{j}}\right)
$$

and

$$
\frac{\partial}{\partial r}=\frac{\partial}{\partial \eta_{j}} \frac{\partial \eta_{j}}{\partial r}+\frac{\partial}{\partial \xi_{j}} \frac{\partial \xi_{j}}{\partial r}=\frac{\partial}{\partial \xi_{j}}+\frac{\partial}{\partial \eta_{j}}
$$

If we define the following quantities in terms of the characteristic variables

$$
\begin{gathered}
\chi_{j}\left(\eta_{j}, \xi_{j}\right) \equiv \psi_{j}(x, r), \\
\hat{\sigma}_{j}\left(\eta_{j}, \xi_{j}\right) \equiv \sigma_{j}(r),
\end{gathered}
$$

and

$$
G_{j}\left(\eta_{j}, \xi_{j}\right) \equiv \sum_{k} \frac{\bar{\nu}_{j}}{\bar{\nu}_{k}} \int_{r}^{\infty} s_{j k}\left(r, r^{\prime}\right) \psi_{k}\left(x, r^{\prime}\right) d r^{\prime}
$$

the light particle transport equation can be written as

$$
\frac{\partial}{\partial \eta_{j}} \chi_{j}\left(\eta_{j}, \xi_{j}\right)-\frac{1}{2 \nu_{j}} \hat{\sigma}_{j}\left(\eta_{j}, \xi_{j}\right) \chi_{j}\left(\eta_{j}, \xi_{j}\right)=-\frac{1}{2 \nu_{j}} G_{j}\left(\eta_{j}, \xi_{j}\right)
$$

where the division by $\nu_{j}$ is well defined since $\nu_{j}>0$ for charged particles. Equation (120) can be solved using the integrating factor 


$$
\hat{\beta}_{j}\left(\eta_{j}, \xi_{j}\right) \equiv \frac{1}{2 \nu_{j}} \int_{\eta_{0}}^{\eta_{j}} \hat{\sigma}_{j}\left(\eta^{\prime}, \xi_{j}\right) d \eta^{\prime}
$$

where $\eta_{0}$ is a free parameter to be chosen later. Equation (120) is now simplified as

$$
\frac{\partial}{\partial \eta_{j}}\left[e^{-\hat{\beta}_{j}\left(\eta_{j}, \xi_{j}\right)} \chi_{j}\left(\eta_{j}, \xi_{j}\right)\right]=-\frac{1}{2 \nu_{j}} e^{-\hat{\beta}_{j}\left(\eta_{j}, \xi_{j}\right)} G_{j}\left(\eta_{j}, \xi_{j}\right)
$$

which can be inverted by integrating from $\eta_{0}$ to $\eta_{j}$. This integration produces

$$
\chi_{j}\left(\eta_{j}, \xi_{j}\right)=e^{\hat{\beta}_{j}\left(\eta_{j}, \xi_{j}\right)} \chi_{j}\left(\eta_{0}, \xi_{j}\right)-\frac{1}{2 \nu_{j}} \int_{\eta_{0}}^{\eta_{j}} e^{\hat{\beta}_{j}\left(\eta_{j}, \xi_{j}\right)-\hat{\beta}_{j}\left(\eta^{\prime}, \xi_{j}\right)} G_{j}\left(\eta^{\prime}, \xi_{j}\right) d \eta^{\prime}
$$

This equation must now be transformed back into the original variables $(x, r)$. The kernel, $G_{j}\left(\eta^{\prime}, \xi_{j}\right)$, is expressed in terms of the original variables by noting that

$$
\begin{aligned}
G_{j}\left(\eta^{\prime}, \xi_{j}\right) & =\sum_{k} \frac{\bar{\nu}_{j}}{\bar{\nu}_{k}} \int_{\frac{\xi_{j}+\eta^{\prime}}{2}}^{\infty} s_{j k}\left(\frac{\xi_{j}+\eta^{\prime}}{2}, r^{\prime}\right) \psi_{k}\left(\frac{\xi_{j}-\eta^{\prime}}{2 \nu_{j}}, r^{\prime}\right) d r^{\prime} \\
& =\sum_{k} \frac{\bar{\nu}_{j}}{\bar{\nu}_{k}} \int_{\frac{\xi_{j}+\eta^{\prime}}{2}}^{\infty} s_{j k}\left(\frac{r+\nu_{j} x+\eta^{\prime}}{2}, r^{\prime}\right) \psi_{k}\left(\frac{r+\nu_{j} x-\eta^{\prime}}{2 \nu_{j}}, r^{\prime}\right) d r^{\prime} .
\end{aligned}
$$

Since the fluence at $x=0$ is known (see the boundary condition in equation (3)), select the free parameter $\eta_{0}=\xi_{j}$ to obtain

$$
\chi_{j}\left(\eta_{0}, \xi_{j}\right)=\psi_{j}\left(\frac{\xi_{j}-\eta_{0}}{2 \nu_{j}}, \frac{\xi_{j}+\eta_{0}}{2}\right)=\psi_{j}\left(0, \xi_{j}\right)=\psi_{j}\left(0, r+\nu_{j} x\right)
$$

The integrating factor $\hat{\beta}_{j}\left(\eta_{j}, \xi_{j}\right)$ is expressed in terms of the original variables $(x, r)$ by noting that $\eta_{0}=\xi_{j}$ has already been chosen, so that

$$
\begin{aligned}
\hat{\beta}_{j}\left(\eta_{j}, \xi_{j}\right) & =\frac{1}{2 \nu_{j}} \int_{\eta_{0}}^{\eta_{j}} \hat{\sigma}_{j}\left(\eta^{\prime}, \xi_{j}\right) d \eta^{\prime} \\
& =\frac{1}{2 \nu_{j}} \int_{\xi_{j}}^{\eta_{j}} \hat{\sigma}_{j}\left(\eta^{\prime}, \xi_{j}\right) d \eta^{\prime} \\
& =\frac{1}{2 \nu_{j}} \int_{r+\nu_{j} x}^{r-\nu_{j} x} \sigma_{j}\left(\frac{r+\nu_{j} x+\eta^{\prime}}{2}\right) d \eta^{\prime} \\
& =-\int_{0}^{x} \sigma_{j}\left(r+\nu_{j} t\right) d t
\end{aligned}
$$

Define

$$
\beta_{j}(r, x) \equiv \int_{0}^{x} \sigma_{j}\left(r+\nu_{j} t\right) d t .
$$


The exponential terms appearing in the integrand of equation (123) can also be written in terms of the original variables by again noting that $\eta_{0}=\xi_{j}$ has been chosen. In this case,

$$
\begin{aligned}
\hat{\beta}_{j}\left(\eta_{j}, \xi_{j}\right) & -\hat{\beta}_{j}\left(\eta^{\prime}, \xi_{j}\right)=\frac{1}{2 \nu_{j}} \int_{\xi_{j}}^{\eta_{j}} \hat{\sigma}_{j}\left(\eta^{\prime \prime}, \xi_{j}\right) d \eta^{\prime \prime}-\frac{1}{2 \nu_{j}} \int_{\xi_{j}}^{\eta^{\prime}} \hat{\sigma}_{j}\left(\eta^{\prime \prime}, \xi_{j}\right) d \eta^{\prime \prime} \\
& =\frac{1}{2 \nu_{j}} \int_{r+\nu_{j} x}^{r-\nu_{j} x} \sigma_{j}\left(\frac{r+\nu_{j} x+\eta^{\prime \prime}}{2}\right) d \eta^{\prime \prime}-\frac{1}{2 \nu_{j}} \int_{r+\nu_{j} x}^{\eta^{\prime}} \sigma_{j}\left(\frac{r+\nu_{j} x+\eta^{\prime \prime}}{2}\right) d \eta^{\prime \prime} \\
& =-\int_{0}^{x} \sigma_{j}\left(r+\nu_{j} t\right) d t+\int_{\frac{\eta^{\prime}+\nu_{j} x-r}{2 \nu_{j}}}^{x} \sigma_{j}\left(r+\nu_{j} t\right) d t \\
& =-\beta_{j}\left(r, \frac{\eta^{\prime}+\nu_{j} x-r}{2 \nu_{j}}\right) .
\end{aligned}
$$

Then equation (123) can be written in terms of the original variables $(x, r)$ as

$$
\begin{gathered}
\psi_{j}(x, r)=e^{-\beta_{j}(r, x)} \psi_{j}\left(0, r+\nu_{j} x\right) \\
-\frac{1}{2 \nu_{j}} \sum_{k} \frac{\bar{\nu}_{j}}{\bar{\nu}_{k}} \int_{r+\nu_{j} x}^{r-\nu_{j} x} \int_{\frac{r+\nu_{j} x+\eta^{\prime}}{2}}^{\infty} e^{-\beta_{j}\left(r^{\frac{\eta^{\prime}+\nu_{j} x-r}{2 \nu_{j}}}\right)_{s_{j k}}\left(\frac{r+\nu_{j} x+\eta^{\prime}}{2}, r^{\prime}\right)} \\
\times \psi_{k}\left(\frac{r+\nu_{j} x-\eta^{\prime}}{2 \nu_{j}}, r^{\prime}\right) d r^{\prime} d \eta^{\prime} .
\end{gathered}
$$

Let

$$
\eta^{\prime}=r-\nu_{j} x+2 \nu_{j} x^{\prime}
$$

then equation (129) becomes

$$
\begin{aligned}
\psi_{j}(x, r)= & e^{-\beta_{j}(r, x)} \psi_{j}\left(0, r+\nu_{j} x\right) \\
& +\sum_{k} \frac{\bar{\nu}_{j}}{\bar{\nu}_{k}} \int_{0}^{x} \int_{r+\nu_{j} x^{\prime}}^{\infty} e^{-\beta_{j}\left(r, x^{\prime}\right)} s_{j k}\left(r+\nu_{j} x^{\prime}, r^{\prime}\right) \psi_{k}\left(x-x^{\prime}, r^{\prime}\right) d r^{\prime} d x^{\prime} .
\end{aligned}
$$

At this time, return to equation (23) and seek to obtain a similar expression for neutrons. For neutral particles, the continuous slowing down term is identically zero, and so the relevant transport equation simplifies to

$$
\left[\frac{\partial}{\partial x}+\sigma_{n}(E)\right] \psi_{n}(x, r)=\sum_{k} \frac{1}{\bar{\nu}_{k}} \int_{r}^{\infty} s_{n k}\left(r, r^{\prime}\right) \psi_{k}\left(x, r^{\prime}\right) d r^{\prime}
$$

The integrating factor defined in equation (127) can be used to invert this equation. Note that for neutrons, equation (127) takes the special form

$$
\beta_{n}(r, x)=x \sigma_{n}(r)
$$

Equation (132) is expressed in terms of this integrating factor as 


$$
\frac{\partial}{\partial x}\left[\psi_{n}(x, r) e^{x \sigma_{n}(r)}\right]=\sum_{k} \frac{1}{\bar{\nu}_{k}} e^{x \sigma_{n}(r)} \int_{r}^{\infty} s_{n k}\left(r, r^{\prime}\right) \psi_{k}\left(x, r^{\prime}\right) d r^{\prime}
$$

Integrate this equation from the boundary at $z=0$ to some arbitrary point in the material at $z=x$, then equation (134) becomes

$$
\psi_{n}(x, r)=e^{-x \sigma_{n}(r)} \psi_{j}(0, r)+\sum_{k} \frac{1}{\bar{\nu}_{k}} \int_{0}^{x} \int_{r}^{\infty} e^{\sigma_{n}(r)(z-x)} s_{n k}\left(r, r^{\prime}\right) \psi_{n}\left(z, r^{\prime}\right) d r^{\prime} d z
$$

Now let

$$
z \equiv x-x^{\prime}
$$

then equation (135) becomes

$$
\psi_{n}(x, r)=e^{-x \sigma_{n}(r)} \psi_{j}(0, r)+\sum_{k} \frac{1}{\bar{\nu}_{k}} \int_{0}^{x} \int_{r}^{\infty} e^{-\sigma_{n}(r) x^{\prime}} s_{n k}\left(r, r^{\prime}\right) \psi_{n}\left(x-x^{\prime}, r^{\prime}\right) d r^{\prime} d z
$$

Upon further inspection, if $j=n$ in equation (131), then $\nu_{n}=0$, and equation (137) is recovered. Therefore, the light particle transport equation can be completely expressed for neutrons and light ions in terms of the Volterra type integral equation as

$$
\begin{aligned}
\psi_{j}(x, r) & =e^{-\beta_{j}(r, x)} \psi_{j}\left(0, r+\nu_{j} x\right) \\
& +\sum_{k} \frac{\bar{\nu}_{j}}{\bar{\nu}_{k}} \int_{0}^{x} \int_{r+\nu_{j} x^{\prime}}^{\infty} e^{-\beta_{j}\left(r, x^{\prime}\right)} s_{j k}\left(r+\nu_{j} x^{\prime}, r^{\prime}\right) \psi_{k}\left(x-x^{\prime}, r^{\prime}\right) d r^{\prime} d x^{\prime} .
\end{aligned}
$$

Equation (138) is the light particle Volterra type integral equation for which numerical procedures will be developed.

\section{Appendix B - Inversion of Heavy Ion Transport Equation}

In this section, the scaled heavy ion transport equation (equation (38) in Section 4)

$$
\left[\frac{\partial}{\partial x}-\nu_{j} \frac{\partial}{\partial r}+\sigma_{j}(r)\right] \psi_{j}(x, r)=\sum_{k>j} \frac{\nu_{j}}{\nu_{k}} \sigma_{j k}(r) \psi_{k}(x, r)
$$

is inverted to obtain a Volterra type integral equation.

Define the characteristic variables

$$
\eta_{j}=r-\nu_{j} x
$$

and

$$
\xi_{j}=r+\nu_{j} x,
$$

along with the functions

$$
\chi_{j}\left(\eta_{j}, \xi_{j}\right)=\psi_{j}(x, r),
$$




$$
\hat{\sigma}_{j}\left(\eta_{j}, \xi_{j}\right)=\sigma_{j}(r)
$$

and

$$
\tilde{\sigma}_{j k}\left(\eta_{j}, \xi_{j}\right)=\sigma_{j k}(r)
$$

so that the differential operator on the left hand side of equation (139) transforms as in the previous section, and one obtains

$$
\frac{\partial}{\partial \eta_{j}} \chi_{j}\left(\eta_{j}, \xi_{j}\right)-\frac{1}{2 \nu_{j}} \hat{\sigma}_{j}\left(\eta_{j}, \xi_{j}\right) \chi_{j}\left(\eta_{j}, \xi_{j}\right)=-\frac{1}{2 \nu_{j}} \sum_{k>j} \frac{\nu_{j}}{\nu_{k}} \tilde{\sigma}_{j k}\left(\eta_{k}, \xi_{k}\right) \chi_{k}\left(\eta_{k}, \xi_{k}\right)
$$

Equation (145) can be solved using the integrating factor

$$
\hat{\beta}_{j}\left(\eta_{j}, \xi_{j}\right) \equiv \frac{1}{2 \nu_{j}} \int_{\eta_{0}}^{\eta_{j}} \hat{\sigma}_{j}\left(\eta^{\prime}, \xi_{j}\right) d \eta^{\prime}
$$

where $\eta_{0}$ is a free parameter to be chosen later. Equation (145) is now simplified as

$$
\frac{\partial}{\partial \eta_{j}}\left[e^{-\hat{\beta}_{j}\left(\eta_{j}, \xi_{j}\right)} \chi_{j}\left(\eta_{j}, \xi_{j}\right)\right]=-\frac{1}{2 \nu_{j}} e^{-\hat{\beta}_{j}\left(\eta_{j}, \xi_{j}\right)} \sum_{k>j} \frac{\nu_{j}}{\nu_{k}} \tilde{\sigma}_{j k}\left(\eta_{k}, \xi_{k}\right) \chi_{k}\left(\eta_{k}, \xi_{k}\right)
$$

which can be inverted by integrating from $\eta_{0}$ to $\eta_{j}$. This integration produces

$$
\chi_{j}\left(\eta_{j}, \xi_{j}\right)=e^{\hat{\beta}_{j}\left(\eta_{j}, \xi_{j}\right)} \chi_{j}\left(\eta_{0}, \xi_{j}\right)-\frac{1}{2 \nu_{j}} \sum_{k>j} \frac{\nu_{j}}{\nu_{k}} \int_{\eta_{0}}^{\eta_{j}} e^{\hat{\beta}_{j}\left(\eta_{j}, \xi_{j}\right)-\hat{\beta}_{j}\left(\eta^{\prime}, \xi_{j}\right)} \tilde{\sigma}_{j k}\left(\eta^{\prime}, \xi_{k}\right) \chi_{k}\left(\eta^{\prime}, \xi_{k}\right) d \eta^{\prime}
$$

Since the fluence at $x=0$ is known (see the boundary condition in equation (3)), we select the free parameter $\eta_{0}=\xi_{j}$ to obtain

$$
\chi_{j}\left(\eta_{0}, \xi_{j}\right)=\psi_{j}\left(\frac{\xi_{j}-\eta_{0}}{2 \nu_{j}}, \frac{\xi_{j}+\eta_{0}}{2}\right)=\psi_{j}\left(0, \xi_{j}\right)=\psi_{j}\left(0, r+\nu_{j} x\right)
$$

The integrating factor $\hat{\beta}_{j}\left(\eta_{j}, \xi_{j}\right)$ is expressed in terms of the original variables $(x, r)$ by noting that $\eta_{0}=\xi_{j}$ has already been chosen, so that 


$$
\begin{aligned}
\hat{\beta}_{j}\left(\eta_{j}, \xi_{j}\right) & =\frac{1}{2 \nu_{j}} \int_{\eta_{0}}^{\eta_{j}} \hat{\sigma}_{j}\left(\eta^{\prime}, \xi_{j}\right) d \eta^{\prime} \\
& =\frac{1}{2 \nu_{j}} \int_{\xi_{j}}^{\eta_{j}} \hat{\sigma}_{j}\left(\eta^{\prime}, \xi_{j}\right) d \eta^{\prime} \\
& =\frac{1}{2 \nu_{j}} \int_{r+\nu_{j} x}^{r-\nu_{j} x} \sigma_{j}\left(\frac{r+\nu_{j} x+\eta^{\prime}}{2}\right) d \eta^{\prime} \\
& =-\int_{0}^{x} \sigma_{j}\left(r+\nu_{j} t\right) d t \\
& =-\beta_{j}(r, x) .
\end{aligned}
$$

The exponential terms appearing in the integrand of equation (148) can also be written in terms of the original variables by again noting that $\eta_{0}=\xi_{j}$ has been chosen. In this case,

$$
\begin{aligned}
\hat{\beta}_{j}\left(\eta_{j}, \xi_{j}\right) & -\hat{\beta}_{j}\left(\eta^{\prime}, \xi_{j}\right)=\frac{1}{2 \nu_{j}} \int_{\xi_{j}}^{\eta_{j}} \hat{\sigma}_{j}\left(\eta^{\prime \prime}, \xi_{j}\right) d \eta^{\prime \prime}-\frac{1}{2 \nu_{j}} \int_{\xi_{j}}^{\eta^{\prime}} \hat{\sigma}_{j}\left(\eta^{\prime \prime}, \xi\right) d \eta^{\prime \prime} \\
& =\frac{1}{2 \nu_{j}} \int_{r+\nu_{j} x}^{r-\nu_{j} x} \sigma_{j}\left(\frac{r+\nu_{j} x+\eta^{\prime \prime}}{2}\right) d \eta^{\prime \prime}-\frac{1}{2 \nu_{j}} \int_{r+\nu_{j} x}^{\eta^{\prime}} \sigma_{j}\left(\frac{r+\nu_{j} x+\eta^{\prime \prime}}{2}\right) d \eta^{\prime \prime} \\
& =-\int_{0}^{x} \sigma_{j}\left(r+\nu_{j} t\right) d t+\int_{\frac{\eta^{\prime}+\nu_{j} x-r}{2 \nu_{j}}}^{x} \sigma_{j}\left(r+\nu_{j} t\right) d t \\
& =-\beta_{j}\left(r, \frac{\eta^{\prime}+\nu_{j} x-r}{2 \nu_{j}}\right) .
\end{aligned}
$$

Then, equation (148) can be written in terms of the original variables as

$$
\begin{array}{r}
\psi_{j}(x, r)=e^{-\beta_{j}(r, x)} \psi_{j}\left(0, r+\nu_{j} x\right) \\
-\frac{1}{2 \nu_{j}} \sum_{k>j} \frac{\nu_{j}}{\nu_{k}} \int_{r+\nu_{j} x}^{r-\nu_{j} x} e^{-\beta_{j}\left(r, \frac{\eta^{\prime}+\nu_{j} x-r}{2 \nu_{j}}\right)} \sigma_{j k}\left(\frac{r+\nu_{j} x+\eta^{\prime}}{2}\right) \\
\times \psi_{k}\left(\frac{r+\nu_{j} x-\eta^{\prime}}{2 \nu_{j}}, \frac{r+\nu_{j} x+\eta^{\prime}}{2}\right) d \eta^{\prime} .
\end{array}
$$

Define

$$
\eta^{\prime} \equiv r-\nu_{j} x+2 \nu_{j} x^{\prime}
$$

then, equation (152) becomes

$$
\begin{aligned}
\psi_{j}(x, r)= & e^{-\beta_{j}(r, x)} \psi_{j}\left(0, r+\nu_{j} x\right) \\
& +\sum_{k>j} \frac{\nu_{j}}{\nu_{k}} \int_{0}^{x} e^{-\beta_{j}\left(r, x^{\prime}\right)} \sigma_{j k}\left(r+\nu_{j} x^{\prime}\right) \psi_{k}\left(x-x^{\prime}, r+\nu_{j} x^{\prime}\right) d x^{\prime} .
\end{aligned}
$$

Equation (154) is the heavy ion Volterra type integral equation for which numerical procedures will be developed. 


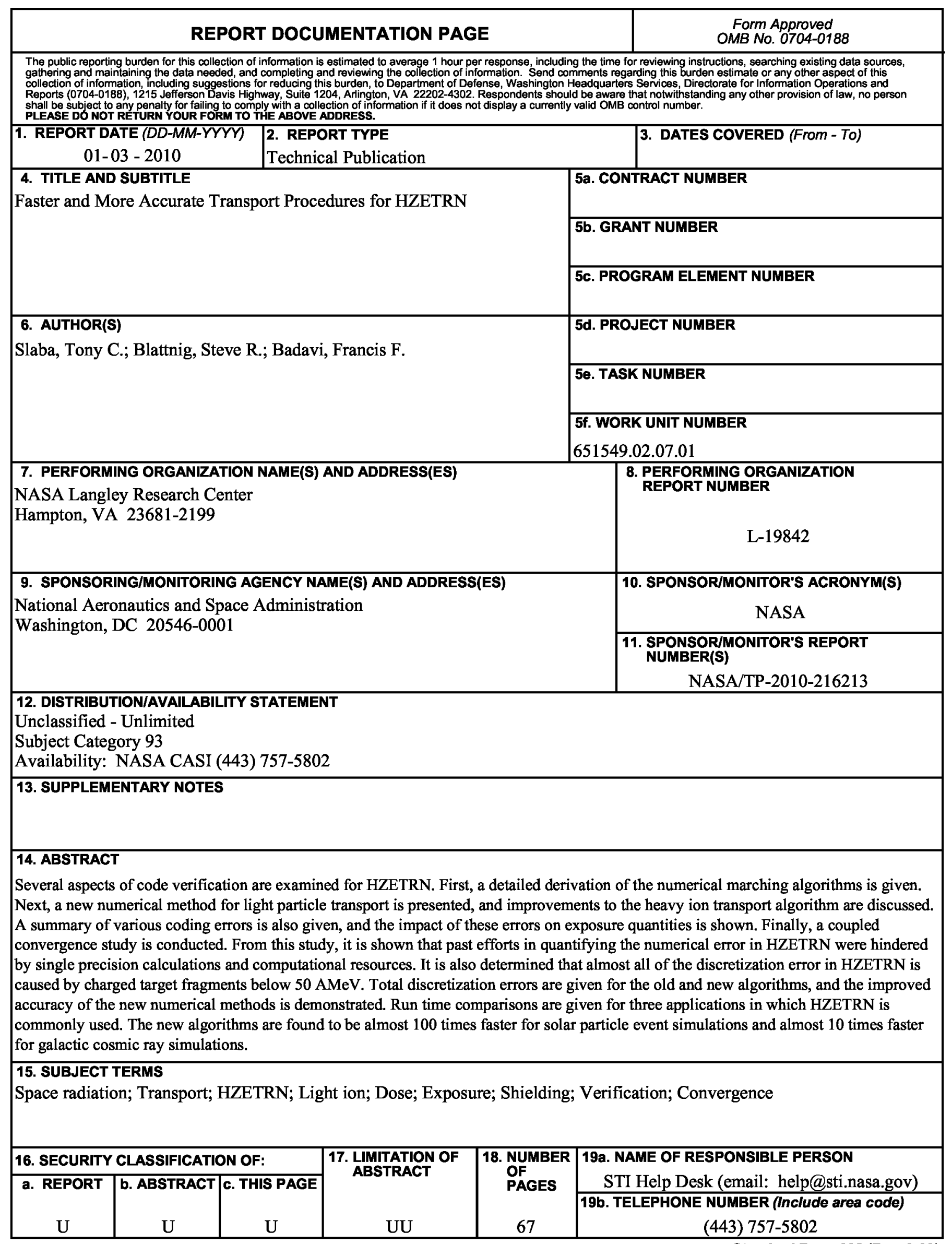

